\title{
Laboratory Directed Research and Development Program FY 2008
}

March 2009 


\section{DISCLAIMER}

This document was prepared as an account of work sponsored by the United States Government. While this document is believed to contain correct information, neither the United States Government nor any agency thereof, nor The Regents of the University of California, nor any of their employees, makes any warranty, express or implied, or assumes any legal responsibility for the accuracy, completeness, or usefulness of any information, apparatus, product, or process disclosed, or represents that its use would not infringe privately owned rights. Reference herein to any specific commercial product, process, or service by its trade name, trademark, manufacturer, or otherwise, does not necessarily constitute or imply its endorsement, recommendation, or favoring by the United States Government or any agency thereof, or The Regents of the University of California. The views and opinions of authors expressed herein do not necessarily state or reflect those of the United States Government or any agency thereof or The Regents of the University of California.

Lawrence Berkeley Laboratory is an equal opportunity employer. 


\author{
Report on \\ Ernest Orlando Lawrence \\ Berkeley National Laboratory
}

\title{
Laboratory Directed Research and Development Program
}

FY 2008

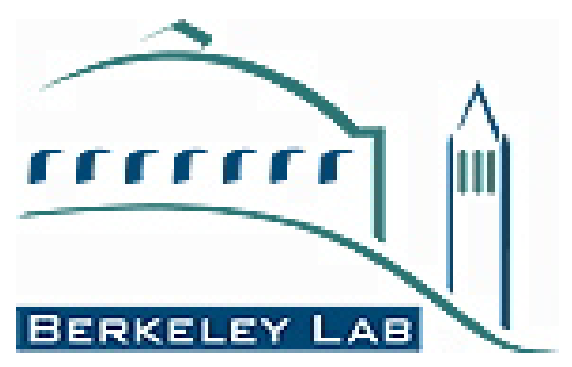

\author{
Ernest Orlando Lawrence \\ Berkeley National Laboratory \\ Berkeley, CA 94720
}

MARCH, 2009

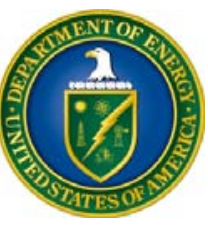





\section{Table of Contents}

Introduction

William Fawley

FEL Concepts for Multiple Independent X-ray Beamlines ...

Alexander Zholents

Gregory Penn

John Corlett

Qing Ji

Ultra-compact Field Desorption Neutron Source for Cancer

Research ......

Thomas Schenkel

Quantum Information Science with Integrated Color Centers in Diamond .....

Csaba Toth

Carl Schroeder

Experimental Demonstration of a Laser-Plasma-Accelerator

William Fawley

Driven Free-Electron Laser

Kem Robinson

Jean-Luc Vay

William Fawley

Weishi Wan

Howard Padmore

Alexander Zholents

et al

Lorentz Compaction of Scales for Ultra-efficient Simulation of Advanced Accelerators (and other systems)...

High Brightness Photocathodes as Electron Sources for FELs ......

Emittance Manipulation and Beam Conditioning for FELs.

Advanced Light Source Division

Elke Arenholz

Soren Prestemon

Jinghua Guo

Alexander Hexemer

et al

Eli Rotenberg

Aaron Bostwick

Steve Kevan

Karsten Horn

David Shapiro

Yiqiong Zhao

Valeriy Yashchuk
Optimization of Flux Pinning Type II Superconductor Based Magnets for Soft X-ray Scattering Applications.

Building In-Situ Electronic Structure Study Capability with Photon-in/ Photon-out Soft X-ray Spectroscopy

Soft X-ray Scattering as a New Probe of Polymer Systems 10

Carbon-Based Nanostructures as a Prototype for New

Electronics

Development of a Laser Goniometer for X-ray and Electron

Diffraction Microscopy

Ultra-high Resolution Optics for Soft X-ray Inelastic Scattering... 13

Chemical Sciences Division

John Arnold

Robert Bergman Jonathan Ellman
Synthetic and Electrochemical Approaches to Metal-Metal

Bonds in Actinides

Conversion of Glycerol and Aromatic Compounds from

Biomass to Major 3- and 6- Carbon Industrial Organic

Compounds 
Hendrik Bluhm

Kevin Wilson

Oliver Gessner

F. Dean Toste

\section{Computing Sciences}

(National Energy Research Scientific Computing Center, and Computational Research Divisions) .. 19

Deb Agarwal

Chin Guok

John Bell

John Bell

Jonathan Carter

Leonid Oliker

John Shalf

Victor Markowitz

Natalia Ivanova

Nikos Kyrpides

Phil Hugenholtz

Doron Rotem

Ekow Otoo

Michael Wehner

Leonid Oliker

John Shalf

David Skinner

Erich Strohmaier

Jon Wilkening

Earth Sciences Division

James Berryman

John Christensen

William Collins

Inez Fung

William Collins

Hoi-Ying Holman

Ping $\mathrm{Hu}$

Sharon Borglin

Christer Jansson
Chemical Reactions at Liquid/Vapor Interfaces Probed by

Photoemission Spectroscopy

Probing Transient Molecular Entanglement Using Femtosecond

High Resolution Delayed-field Coincidence Imaging

Metal Nitrosyl Complexes and Catalytic C-H Bond

Functionalization

Structured, Adaptive Mesh Refinement Method for Multiphase

Reactive Transport in Groundwater

Low Order Models for Ballistic Charge Transport in Nanoscale

Devices

Enhancing the Effectiveness of Manycore Chip Technologies

for High-End Computing

Integrated Microbial Community Genomes Data Management System

Energy-Smart Disk-Based Mass Storage System.....

Holistic Approach to Energy Efficient Computing Architecture....

Integrated Performance Monitoring of Grid and HPC

Workloads

Reference Benchmarks for the Dwarfs (Algorithms)

Extended First Order System Least Squares Finite Elements.

28
Applications of Adjoint Field Methods and Time-Reversal Data Processing to Inverse Problems in Electromagnetics, Seismics, and Ultrasonics.

Lattice-Boltzmann Investigations of Isotopic Fractionation During Mineral Precipitation

Integrated Earth Systems Climate Modeling and Analysis

Global Methane Cycle and Climate Change....

Compositional and Functional Analysis of Cell-Wall During Metal-Bacterial Interactions.

Regulation of Carbon Partitioning in Grasses 
Janet Jansson

Seiji Nakagawa

Andrea Cortis

Curtis Oldenburg

William Riley

Norman Miller

Dale Perry
Microbiomics of Complex Microbial Communities in

Environmental Samples

New Technology for Permeability Enhancement for Natural

Gas Extraction in Tight Reservoirs

Coupled Modeling of Hydrology, Nutrient Cycling, and Vegatation: Applications to Water Quality and Water Balance.....

Design and Properties of Materials Involving Elements with

High Neutron Cross-Sections that are Useful in Neutron

Detection and Related Neutron Technologies

Environmental Energy Technologies Division

Hugo Destaillats

Phil Haves

Thomas Kirchstetter

Mark Levine

Melissa Lunden

James McMahon

et al

Mark Mendell

Francis Rubinstein

Mike Sohn

Ashok Gadgil

Michael Wetter
Understanding the Chemistry of Innovative Air Cleaning

Technologies

Development of a Building Operating Platform

Soot in Ice: Does Soot Enhance the Melting of Ice?

China's Energy Future: Changes in Energy Intensity

Novel Techniques to Characterize Secondary Organic Aerosols Formed From Gas-phase Volatile Organic Compounds

Integrated Decision Support Tool for Joint Optimal Control of Energy and Water Systems under Uncertainty

Using New Microbial Assays to Characterize Dampness-related Exposures: Combining Molecular Biology with Building, Aerosol, and Health Science

Using IP Telephony and Wireless Technologies to Extend the Reach of Conventional Building Automation Systems

Software for Integrated Analysis of Sensor Data for Advanced Energy Controls

Building Informatics Environment Enabling Rapid Prototyping and Model Extraction for Building Automation Systems...

Jonathan Eisen

Computational and Experimental Testing of Methods for

Binning Sequences from Metagenomic Studies

Life Sciences Division

Gareth Butland

Functional Interactomics: Integrating Physical and Functional Interaction Networks

Stephen Derenzo

Heavy-atom Ultra-fast Scintillator for Time-Of-Flight Positron Emission Tomography

Bo Hang

Interaction of Fragile X Mental Retardation Protein with Thymine-DNA Glycosylase: Implication in the Molecular Mechanism of Fragile X-Syndrome

Terumi Kohwi-

Genome Organizer in Carcinogenesis

Shigematsu et al

Andrew Wyrobek Francesco Marchetti
Expression Profiling of Radiation and Cancer Susceptibility Genes ..... 


\author{
Ali Javey \\ Blandine Jérôme \\ Jeffrey Long \\ Robert Ritchie
}

Eduardo Saiz

Irfan Siddiqi

Dan Stamper-Kurn

Jeff Urban

Taleb Mokari et al

Junqiao $\mathrm{Wu}$

Ting Xu
Integration of Synthetic Nanomaterials for High Performance, Robust, and Flexible Circuitry ...................................................... 55

Transport in Thin Polymer Films ................................................... 56

Conducting Metal-Organic Frameworks ...................................... 57

Fracture of Cortical Bone: Mechansims Underlying the Origins of Toughness in Hard Mineralized Tissues ..................................... 58

Three Dimensional Printing of Ceramics with Submicron

Resolution

Quantifying the Quantum Backaction of a Non-Linear

Dispersive Measurement .....

Two-Element Ultracold-Atom Quantum Simulator of Materials ...

Understanding the Electronic Energy Level Alignment at

Nanoscale Interfaces

Transition-Metal-Doped GeTe Nanowires as a Single-Phase

Multiferroic System..... 63

Hierarchical Assemblies of Peptide-Polymer Conjugates.

5

6


Christian Bauer

Soft-collinear Effective Theories for Collider Physics.

Maurice Garcia-Sciveres

Detectors.

Murdock Gilchriese

Carl Haber

Nikhil Padmanabhan

Martin White

David Schlegel

Natalie Roe

Stephen Holland

Nick Palaio

David Schlegel

George Smoot

Alexie Leauthaud
Development of Multi-Modular Assemblies with Reduced Material and Services for Specifications at Future Particle Tracking

Calibrating Baryon Acoustic Oscillations for Future Dark Energy Experiments.

High-Yield CCD Process Development at the LBNL

MicroSystems Lab.

Baryon Oscillations and Dark Energy: Prototyping Instruments ... 79

Decoding Dark Energy with Weak Gravitational Lensing ............ 80

Cross-Divisional.

Manfred Auer

Jan Liphardt

Phil Hugenholtz

Stephen Holbrook Jennifer Doudna

Jill Banfield

Steven Yannone

Seung-Wuk Lee

Lee Schroeder

et al
Multi-scale Imaging of Termite Hind Gut Microbial Biofilm

Do CRISPR Regions of Bacteria and Archaea Constitute an RNAi Based Immune System?

Fabrication of Photovoltaic Devices Using Nanostructured Biomaterials

Coupled Process Models, Separations, Monitoring for Advanced Nuclear Fuel Cycles..... 



\section{Introduction}

The Ernest Orlando Lawrence Berkeley National Laboratory (Berkeley Lab or LBNL) is a multi-program national research facility operated by the University of California for the Department of Energy (DOE). As an integral element of DOE's National Laboratory System, Berkeley Lab supports DOE's missions in fundamental science, energy resources, and environmental quality. Berkeley Lab programs advance four distinct goals for DOE and the nation:

- To perform leading multidisciplinary research in the computing sciences, physical sciences, energy sciences, biosciences, and general sciences in a manner that ensures employee and public safety and protection of the environment.

- To develop and operate unique national experimental facilities for qualified investigators.

- To educate and train future generations of scientists and engineers to promote national science and education goals.

- To transfer knowledge and technological innovations and to foster productive relationships among Berkeley Lab's research programs, universities, and industry in order to promote national economic competitiveness.

Berkeley Lab's research and the Laboratory Directed Research and Development (LDRD) program support DOE's Strategic Themes that are codified in DOE's 2006 Strategic Plan (DOE/CF-0010), with a primary focus on Scientific Discovery and Innovation. For that strategic theme, the Fiscal Year (FY) 2008 LDRD projects support each one of the three goals through multiple strategies described in the plan. In addition, LDRD efforts support the four goals of Energy Security, the two goals of Environmental Responsibility, and Nuclear Security (unclassified fundamental research that supports stockpile safety and nonproliferation programs). The LDRD program supports Office of Science strategic plans, including the 20year Scientific Facilities Plan and the Office of Science Strategic Plan. The research also supports the strategic directions periodically under consideration and review by the Office of Science Program Offices, such as LDRD projects germane to new research facility concepts and new fundamental science directions.

Berkeley Lab LDRD program also play an important role in leveraging DOE capabilities for national needs. The fundamental scientific research and development conducted in the program advances the skills and technologies of importance to our Work For Others (WFO) sponsors. Among many directions, these include a broad range of health-related science and technology of interest to the National Institutes of Health, breast cancer and accelerator research supported by the Department of Defense, detector technologies that should be useful to the Department of Homeland Security, and particle detection that will be valuable to the Environmental Protection Agency.

The Berkeley Lab Laboratory Directed Research and Development Program FY2008 report is compiled from annual reports submitted by principal investigators following the close of the fiscal year. This report describes the supported projects and summarizes their accomplishments. It constitutes a part of the LDRD program planning and documentation process that includes an annual planning cycle, project selection, implementation, and review.

The Berkeley Lab LDRD program is a critical tool for directing the Laboratory's forefront scientific research capabilities toward vital, excellent, and emerging scientific challenges. The program provides the resources for Berkeley Lab scientists to make rapid and significant contributions to critical national science and technology problems. The LDRD program also advances Berkeley Lab's core competencies, foundations, and scientific capability, and permits exploration of exciting new opportunities. All projects are work in forefront areas of science and technology. Areas eligible for support include the following:

- Advanced study of hypotheses, concepts, or innovative approaches to scientific or technical problems;

- Experiments and analyses directed toward "proof of principle" or early determination of the utility of new scientific ideas, technical concepts, or devices; and

- Conception and preliminary technical analyses of experimental facilities or devices.

The LDRD program supports Berkeley Lab's mission in many ways. First, because LDRD funds can be allocated within a relatively short time frame, Berkeley Lab researchers can support the mission of the Department of Energy (DOE) and serve the needs of the nation by quickly responding to forefront scientific problems. Second, LDRD enables Berkeley Lab to attract and retain highly qualified scientists and to support their efforts to carry out worldleading research. In addition, the LDRD program also supports new projects that involve graduate students and postdoctoral fellows, thus contributing to the education mission of Berkeley Lab.

Berkeley Lab has a formal process for allocating funds for the LDRD program. The process relies on individual 
scientific investigators and the scientific leadership of Berkeley Lab to identify opportunities that will contribute to scientific and institutional goals. The process is also designed to maintain compliance with DOE Orders, in particular DOE Order 413.2B (dated April 19, 2006). From year to year, the distribution of funds among the scientific program areas changes. This flexibility optimizes Berkeley Lab's ability to respond to opportunities.

Berkeley Lab LDRD policy and program decisions are the responsibility of the Laboratory Director. The Director has assigned general programmatic oversight responsibility to the Deputy Director. Administration and reporting on the LDRD program is supported by the Directorate's Office for Planning and Development. LDRD accounting procedures and financial management are consistent with the Laboratory's accounting principles and stipulations under the contract between the University of California and the Department of Energy, with accounting maintained through the Laboratory's Chief Financial Officer.

In FY2008, Berkeley Lab was authorized by DOE to establish a funding ceiling for the LDRD program of $\$ 21.0 \mathrm{M}$ including General \& Administrative (G\&A) overhead, which equated to $4.0 \%$ of Berkeley Lab's FY2008 projected operating and capital equipment budgets. This funding level was provided to develop new scientific ideas and opportunities and allow the Berkeley Lab Director an opportunity to initiate new directions. Budget constraints limited available resources, however, so a little more than $\$ 17.7 \mathrm{M}$ was expended for operating and almost $\$ 0.6 \mathrm{M}$ for capital equipment (3.19\% of actual Berkeley Lab FY2008 operating and equipment costs).

In FY2008, scientists submitted 170 proposals, requesting about $\$ 30.8 \mathrm{M}$ in funding prior to assessing laboratory overhead. Eighty-four projects were funded, with awards ranging from $\$ 55 \mathrm{~K}$ to $\$ 746 \mathrm{~K}$. These projects are identified in the Table of Contents. 


\section{Accelerator and Fusion Research Division}

\section{LB07015}

FEL Concepts for Multiple Independent X-ray Beamlines

Principle Investigators: William Fawley, Alexander Zholents, Gregory Penn, John Corlett

Project Description

We propose to address fundamental issues and develop concepts for a next generation, FEL-based light source facility at LBNL. To meet diverse interest of the experimentalist we consider an array of free electron lasers (FELs) where each FEL is tailored to individual experimental needs for average and peak brightness, pulse repetition rate, resolving power, and wavelength. Thus the entire ensemble of FELs will provide a high degree of flexibility for choosing photon energy, $\mathrm{x}$-ray pulse duration, $\mathrm{x}$ ray signal bandwidth and polarization. A variety of FEL configurations such as SASE, HGHG, self-seeded and seeded by HHG signal will be studied. The electron beam switch yard distributing electron bunches into various FELs will be designed.

\section{Accomplishments}

The FEL studies during the previous year of this LDRD indicated that FEL performance critically depends on the electron beam characteristics and excellent results can be achieved using the electron beam with the following parameters: electron beam energy of $2.4 \mathrm{GeV}$, electron beam energy spread of $\sim 100 \mathrm{keV}$, normalized electron beam emittance of $1 \mathrm{~mm}$-mrad and flexible electron bunch length in the range from approximately 600 fs to approximately 10 fs for electron bunch charge of $800 \mathrm{pC}$ and 15 $\mathrm{pC}$ correspondently. This year we focused on a problem of the distribution of the electron bunches with above listed parameters provided by linac at a $1 \mathrm{MHz}$ repetition rate into the array of ten FELs. Particular attention was given to a problem of preserving small electron bunch emittance and energy spread while it propagates from the end of the linac to the beginning of the undulator chain of each individual FEL, i.e. a rather long beamline that includes the kicker, the septum magnet and a set of bending magnets and quadrupoles and that separates the electron bunches from the linac and diverts them into the specific FEL. Equally important was to find the arrangement for the entire switch yard that will allow a convenient use of the BEVATRON site for at least ten undulator chains and a large number of experimental end stations.

Over the course of this work we found that the best performance can be achieved when each beamline is build from two isochronous triple-bend-like achromats connected by a simple FODO section. The electron microbunching and energy modulation due to combined effect of longitudinal space charge and coherent synchrotron radiation was the smallest in this case. Interestingly, the microbunching was rather strong when each achromat was not isochronous, but two achromats together were isochronous. These results were first obtained using linear theory of the microbunching instability and were later confirmed with macroparticle simulations. The first achromat includes a kicker and a septum magnet. A $2 \mathrm{~m}$ long stripline with a narrow $8 \mathrm{~mm}$ gap will be used for a kicker. A novel pulse generator commercially available form FID Technology will provide 15 $\mathrm{kV}, 10 \mathrm{~ns}$ pulses at a $100 \mathrm{kHz}$ repetition. A series of 10 kickers each operating at 100 $\mathrm{kHz}$ repetition rate will distribute the incoming electron bunches from the linac into 10 individual FELs. 


\section{Ultra-compact Field Desorption Neutron Source for Cancer Research \\ Principal Investigator(s): Qing Ji}

\section{Project Description}

The objective of this proposed project is to develop and construct a proof-principle ultracompact, field-desorption neutron generator for cancer research, using a field desorption technique. The generator can be small enough to be housed in a catheter, which allows insertion of the neutron source into the human body and, by adding MRI capability, can be placed at the exact location of the tumors.

The device can be preloaded with pure deuterium, tritium gas, or a mixture, depending on the desired neutron energy and spectrum. For the case of the D-D reaction, deuterium ions, $\mathrm{D}^{+}$, are generated by electrostatic field desorption of deuterium adsorbed on coated tip surfaces. They are then accelerated to $\sim 80 \mathrm{keV}$ when reaching the target surface where the nuclear reactions take place to produce neutrons. By powering on and off, the neutrons can be produced on-demand, thus eliminating expensive shielding for the protection of clinical personnel and patients when radioactive neutron sources are involved. The neutron yield from this type of system is proportional to the number of tips one can pack into the source. With a tip density of $10^{4} / \mathrm{mm}^{2}$, the estimated D-D neutron yield is approximately $10^{5} \mathrm{n} / \mathrm{s}$. Manufacturing of these tips requires microfabrication.

\section{Accomplishments}

We've upgraded our fabrication processes to accommodate 6" wafers, which will allow us to achieve ten times higher tip density by using DUV lithography system. To improve the yield of the tungsten-coated silicon field desorption tips, a two-mask process for microfabrication has been developed. Development of this microfabrication process involved many tests for a number of technical process steps, the specific results of which are part of an invention disclosure and published paper. Compared with previous batch of process (FY '07) in which the density of the tips is approximately $1.6 \times 10^{4} / \mathrm{mm}^{2}$, the number of tips that can be packed in a same chip increases by a factor of ten.

Experimental results from the field emitter tips yield a measurable positive current in the nano-ampere range. However, the ion current is not stable and fluctuates during the period of measurement. 
Quantum information science with integrated color centers in diamond Principal Investigator: Thomas Schenkel, AFRD, LBNL

Project description:

Color centers in diamond, like the nitrogen-vacancy center, exhibit amazing coherence properties, with spin lifetimes approaching milliseconds at room temperature. Color centers promise to revolutionize quantum information technology and our fundamental understanding of quantum coherence when they can be integrated into devices. To date, studies have been limited to uncontrolled, randomly distributed centers, and the coherence properties of many promising centers have not been characterized for lack of efficient center formation. We are forming arrays of color centers with unprecedented spatial resolution (few $\mathrm{nm}$ ) by ion implantation with scanning probe alignment and integrate center arrays into opto-electronic devices. We are also developing processes for efficient center formation and characterize coherence properties of new center systems.

Our unique setup for ion implantation with scanning probe alignment allows nondestructive imaging, alignment and ion placement with nanometer resolution and is ideally suited for the formation of color center arrays in diamond samples (which are usually small and rare). Single ion placement is enabled by the sensing of induced charge from single ion impacts. Samples are selected from commercial supplies of synthetic diamonds with ultra-low nitrogen content, and we explore use of nano-crystalline diamond films on silicon. We make heavy use of the Imaging Facility at the Molecular Foundry for array characterization and collaborate very closely with Prof. Awschalom (UCSB) on the mapping and control of coherence properties using confocal laser spectromicroscopy, and optically detected magnetic resonance.

Following first center array formation in the 1st six month of the project, we succeeded in formation of arrays with $80 \mathrm{~nm}$ dot sizes (Fig 1). First results from coimplantation of argon ions indicate enhanced NV-center formation under optimized vacancy densities. We are now iterating annealing and optical characterization to find the optimal conditions for efficient NV-center formation. Formation of isolated 15-NV centers in ultra-pure diamond enabled detailed spectroscopic studies of the excited state structure of the NV-center that were published in Phys. Rev. Lett.

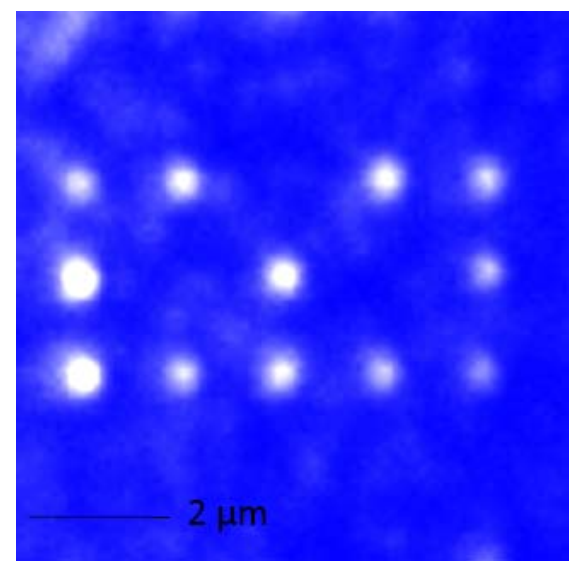

Figure 1: Pattern of NV- centers in ultra-pure, synthetic diamond, formed by ion implantation with scanning probe alignment. Image, courtesy of Greg Fuchs, UCSB. The NV-centers are placed into $80 \mathrm{~nm}$ wide dots. The imaging resolution in the confocal, photoluminescence microscope is about $400 \mathrm{~nm}$. 


\section{LDRD - LPADFEL - FY2008 report \\ Experimental demonstration of a laser-plasma-accelerator driven free-electron laser Principle Investigator(s): Csaba Toth, Carl Schroeder, William Fawley, Kem Robinson}

\section{Project Description}

The goal of this project is the demonstration and development of a free-electron laser (FEL) driven by the laser wakefield accelerator (LWFA) currently operating in AFRD's laser-plasma accelerator facility, the LOASIS Laboratory. The LDRD proposal consists of two stages: (1) The LWFA electron beam will be coupled to a $2.18 \mathrm{~cm}$-period undulator and the synchrotron radiation from one 66-period undulator section will be used to diagnose and characterize the LWFA electron beam. The intrinsic synchronization between the laser, electron beam, and radiation will be demonstrated. (2) Gain will be demonstrated via self-amplified spontaneous emission (SASE) in the XUV wavelength range. Fluxes on the order of $10^{13}$ photons/pulse are anticipated at saturation due to the high current $(>10 \mathrm{kA})$ of the LWFA beams. The LWFA-FEL will constitute a new class of compact laser-driven FEL [in which the conventional accelerator (10-100 $\mathrm{m}$ length) is replaced by a GeV-class laser-plasma accelerator (several $\mathrm{cm}$ )] producing ultra-short $(<50 \mathrm{fs}) \mathrm{XUV}$ pulses with high peak flux.

\section{Accomplishments}

We have achieved significant results in five important areas required for experimental demonstration of a LWFA-driven FEL:

(1) Improved the electron beam quality of the LWFA via installing an optical prepulse contrast filter in the laser beam (which generates the electron beam) and enhanced the pointing stability via a mirror alignment feedback system. We have also designed and built a hexapod-based capillary support to increase flexibility and to improve stability.

(2) In the laser/electron beam separation area we have designed and assembled a waterjet test stand. Initial wavefront measurements show sufficient flatness for laser reflection. We have also explored collaboration on liquid jet design with Argonne National Lab.

(3) As the first step of the undulator commissioning at LBNL, we have transferred the THUNDER undulator from Boeing to Berkeley. Hall-probe magnetic measurements performed on the undulator sections in order to fine-tune the magnetic field structure of the undulator. To house the undulator, a radiation shielded experimental cave has been constructed - occupation is expected in Jan 2009.

(4) We have designed the XUV spectrometer, procured the special optical grating, and purchased the appropriate diagnostic CCD camera. The final design of the housing of the spectrometer was completed and manufacturing is planned to commence by early 2009.

(5) The expected emittance evolution and matching of electron beam into undulator was examined using the high performance simulation code GPT. Using the FEL code GINGER, the focusing of the e-beam into the undulator has been modeled and the expected FEL output calculated. The spontaneous radiation output sensitivity to energy spread and emittance was also calculated using the code SPECTRA. 


\section{Lorentz Compaction of Scales for Ultra-Efficient Simulation of Advanced Accelerators (and Other Systems). \\ Principal Investigators: Jean-Luc Vay, William Fawley}

\section{Project Description}

The purpose of this project is to speed-up by orders of magnitude a class of first-principle computer simulations of complex physical problems, by recasting them into a Lorentz boosted frame of reference. The design and understanding of modern experimental devices relies increasingly upon detailed computer simulation, but despite the awesome progress in the last decades in computer power and algorithmic developments, some problems remain extraordinarily difficult to model accurately. One such problem is laser wakefield acceleration (LWFA). Modeling 10-GeV stages for the LOASIS BELLA proposal in one-dimension using the Particle-In-Cell methods in the laboratory frame demanded as much as 5,000 processor-hours. A calculation of the same problem in a Lorentz boosted frame used only 3 processor-hours, a more than 1,500x speed-up. The new method would allow for 3-D simulation of this problem, which is ultimately needed but is currently beyond the state of the art using the conventional method.

During the course of this project, we will: (1) demonstrate, with actual 2-D or 3-D computer simulations of problems relevant to LWFA, electron clouds (EC) and free electron lasers (FEL) physics problems, orders of magnitude speed-up from a calculation in the laboratory frame to a calculation in the optimum Lorentz frame of reference; (2) for each targeted application, determine the range of validity for the Lorentz-frame simulation results by comparison to laboratory frame simulations and/or well-known analytic results; explore what additional physics become visible via use of the boosted frame approach (3) explore ways to perform calculations at optimum efficiency, regardless of the choice of the frame of reference; (4) explore other scientific applications which might benefit.

\section{Accomplishments}

We showed that the proper cancellation of electric and magnetic field contributions in the Lorentz force is critical to the modeling of some relativistic systems. We demonstrated that the ubiquitous Boris particle pusher does not preserve this property, derived a new particle pusher which does while retaining the other properties of the Boris pusher. We also derived a procedure for solving the fields which retains electrostatic, magnetostatic, and inductive field effects in the direction of the mean velocity of the species, is fully explicit and simpler than the full Darwin approximation. The effectiveness of the two new algorithms was demonstrated in a 3-D Lorentz boosted frame calculations of electron cloud effects.

We also applied the boosted-frame formalism to the modeling of short wavelength FEL. The results indicate that performing electromagnetic simulation in the ponderomotive frame saves orders of magnitude in CPU time over doing the same problem in the lab frame without obvious loss of physics. For most FEL problems of interest at sub-IR wavelengths, however, the boosted frame approach is still orders of magnitude slower than the eikonal method commonly used in FEL simulation. Nonetheless, in some instances, the boosted frame approach allows examination of effects such as matching ramps and sidebands associated with sub-harmonic bunching that are difficult or impossible to study with eikonal codes. Further improvements (e.g., multiple undulator-period averaging) may further increase the efficiency of the boosted frame method.

We presently are applying the method to other accelerator problems, and are examining some fundamental numerical issues regarding its application to laser wakefield acceleration. 


\section{High Brightness Photocathodes as Electron Sources for FELs Principal Investigator(s): Weishi Wan, Howard Padmore}

\section{Project description}

Free Electron Lasers (FELs) offer instantaneous flux and brightness 10 orders of magnitude higher than a $3^{\text {rd }}$ generation synchrotron source like the ALS. They offer in addition pulse lengths of fsecs or less in comparison to the $100 \mathrm{psec}$ of a conventional synchrotron. However, in order to operate, the electron beam has to occupy a very small phase space volume, and, in order to do this, presently operating and planned FELs start with a relatively hot beam from a laser driven photocathode gun, and compress its phase volume by acceleration to high energy. In addition, present day cathodes have limited quantum efficiency, and so, limited by available laser power, FELs typically have low repetition rates. We are addressing these issues by investigation of a new generation of photocathodes, that have engineered electronic properties. The aim is to develop a cathode that produces a beam with very low transverse energy spread; this will enable higher photon energies from a FEL, or simply reduce its scale. In addition, we aim to increase the quantum efficiency to the point at which conventional laser technology can provide enough pulse power to operate at $\mathrm{MHz}$ repetition rate. Successful development of photocathodes would result in reduced cost and technical risk, and enhanced opportunities, for new FEL technical approaches.

On the experimental side, we have used the technique of angle resolved photoemission spectroscopy (ARPES) to investigate copper photocathodes. We have finished setting up the apparatus in our own lab and obtained good photoemission data from $\mathrm{Cu}(111)$. On the theoretical side, we have used a direct N-body simulation code to study the stochastic Coulomb scattering among the electrons to predict the final emittance of the electron beam from the photocathode and have gained useful insight for obtaining the optimal beam.

\section{Accomplishments}

We have finished setting up our lab and obtained quality data from $\mathrm{Cu}(111)$. We confirmed and improved upon our previous result that photo-current at the very low photon energies typically used is dominated by surface states. These results doen in collaboration with the Parmigani group have been published in Applied Physics Letters (93, 183505 (2008)). The emittance deduced from the experimental data agrees quantitatively with the theoretical prediction. This directly has led to a prediction of the minimum transverse momentum, and to a direction for producing lower emittance though use of other crystalline surfaces. On the theoretical side, we have obtained much more detailed understanding of the effect of the stochastic Coulomb scattering on the electron beam. Work has also started on design of a system for Cs2Te production; this is a high quantum yield photoemitter that is a candidate for high rep rate FELs. In addition we have developed a theoretical description of how light can be absorbed in nanometer sized cavities on a metal surface. This can result in complete absorption even in a completely free electron-like material. This greatly widens the possible choices of cathode mnaterials. Work is underway to fabricate such a nano-engineered surface in Aluminum, using state of the art electron and ion beam lithography. This work holds the promise of producing highly robust, low emittance and efficient cathodes. 


\section{Emittance Manipulation and Beam Conditioning for FEL's Principle Investigators: John Corlett, Steve Lidia, Gregory Penn, Ji Qiang, Marco Venturini, Alexander Zholents}

\section{Project Description}

The purpose of the project is to design electron beam emittance control and manipulation techniques for application in a low-energy accelerator feeding several independent FEL beamlines, which could be built at LBNL. Implementation of anticipated techniques would help to obtain a high brightness electron beam in a cost effective facility where control of the electron beam quality will be gained in the electron gun and maintained through the whole accelerator.

\section{Accomplishments}

Building upon knowledge regarding collective effects detrimental to the brightness of the electron beam gained in the previous year of this LDRD and also utilizing previously developed powerful computational tools capable to reliable predictions of the electron beam parameters even when instability itself develops out of the shot noise of electrons, we performed an exemplarily studies of the electron beam accelerator suitable for the electron beam delivery to the array of soft x-ray free electron lasers. Over the course of these studies we have carried out so far the largest scale beam dynamics simulations in accelerator community using multi-billion macro-particle tracking and obtained a clear understanding regarding the machine configuration that works the best (see Figure 1). As a result, we have demonstrated that the bright electron beam with high peak current of $\sim 1$ $\mathrm{kA}$, small relative energy spread of $\sim 10^{-5}$ and small normalized emittance of $\sim 1 \mathrm{~mm}$-mrad can be produced at the end of the linear accelerator with the electron beam energy of 2.4 $\mathrm{GeV}$. We also established the flexibility of the machine by simulating its ability to deliver the electron bunches with $0.8 \mathrm{nC}$ charge and $\sim 600$ femtosecond bunch length suitable for use in the free electron lasers, and also electron bunches with $0.2 \mathrm{nC}$ charge and $\sim 80$ femtosecond bunch length. We reported results of our work at several workshops and conferences and already published them or submitted them for publication (see list of publications and presentations).

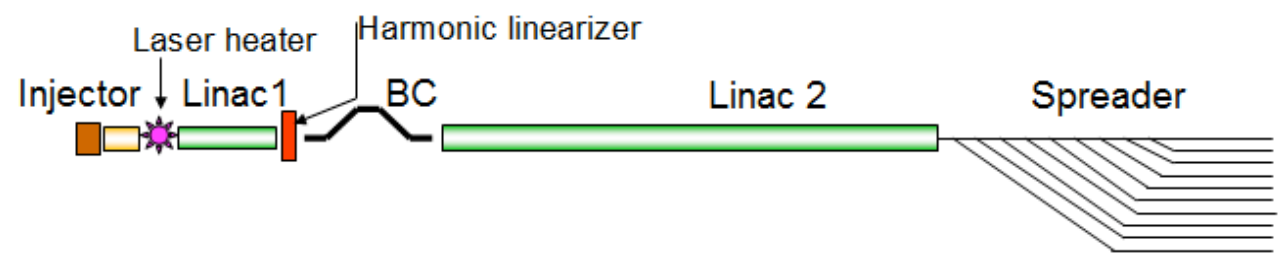

Figure 1. A schematic of the accelerator. Elements of the machine include the injector, laser heater, first linac (Linac1), harmonic linearizer linac, bunch compressor (BC), second linac (Linac2), electron beam switch yard (Spreader) distributing electron bunches into array of free electron lasers (not shown in the picture). 


\section{Advanced Light Source Division}

LB08001

Optimization of flux pinning in type II superconductor based magnets for soft x-ray scattering applications

Principal Investigator(s): Elke Arenholz, Soren O. Prestemon

\section{Project Description}

The delicate balance between spin, charge, lattice, and orbital degrees of freedom in transition metal oxides leads to complex phase diagrams including superconducting, ferro- and antiferromagnetic as well as charge and orbital ordered phases. Soft x-ray scattering provides novel information about charge and orbital ordering in complex oxides but the impact of an external magnetic field on the ordered phases is largely unexplored. This is mainly due to the experimental difficulties associated with providing sufficiently strong magnetic fields, i.e. several $\mathrm{T}$, at the sample surface without limiting the scattering geometries significantly. It is crucial in soft $\mathrm{x}$-ray scattering experiments that the sample be illuminated by an x-ray beam at an angle between $\sim 1^{\circ}$ (very grazing incidence) to $90^{\circ}$ (normal incidence). The scattered x-ray signal is measured either in a backscattering or forward scattering geometry close to the direction of the incoming x-ray beam, i.e. $\sim 1^{\circ}$ to $180^{\circ}$ to the incoming radiation, or any angle in-between. Ideally, a magnet providing an external field in a scattering experiment is located behind or in a plane with the sample surface. Moreover, to maintain a fixed sample/field geometry for different $x$-ray angles of incidence in scattering experiments the field has to be rotated together with the sample. For FY 2008, we proposed to use flux pinning in type II superconductors to create a material with permanent magnet characteristics at low temperatures but providing significantly higher fields. Mounting the superconductor behind the sample in soft $\mathrm{x}$ ray scattering experiments will allow measurements in significant magnetic fields while providing maximum flexibility in the choice of scattering geometry.

\section{Accomplishments}

We developed a characterization set up that allowed applying external magnetic fields of up to $5 \mathrm{~T}$ to type II superconductors held at temperatures between $5 \mathrm{~K}$ and $300 \mathrm{~K}$ under high vacuum conditions. An in-vacuum Hall probe was used to characterize the flux in the superconductor during field cooling and after the external field was reduced to zero. Y-Ba-Cu-O (YBCO) discs (20 mm diameter, $8 \mathrm{~mm}$ thick) provided by R. Weinstein, University of Houston, were identified as most suitable materials for our soft $\mathrm{x}$-ray scattering applications since they exhibit the relevant characteristics:

$+80 \%$ of the field applied during field cooling is reliably pinned in the YBCO discs by defects after removal of the external field at $5 \mathrm{~K}$.

+ At $60 \mathrm{~K}$ fields exceeding $8 \mathrm{~T}$ have been pinned in the YBCO discs by R. Weinstein.

+ A specific field value can be obtained by appropriately choosing applied cooling field and field cooling temperature, i.e. the temperature at which the external field is ramped to zero after the field cooling process.

+ Flux creep, i.e. the loss of pinned flux due to thermal fluctuations, is less than $1 \%$ in 3 hours at $5 \mathrm{~K}$ and can be completely eliminated by lowering the temperature in zero applied field, after the field cooling process is completed.

+ The pinned flux is stable within $1 \%$ for temperatures not exceeding the field cooling temperature which is relevant for temperature dependent studies of complex oxides.

Our findings will form the basis for the development of robust $\theta / 2 \theta$ diffractometer to allow novel soft x-ray scattering experiments on complex oxides in high external magnetic fields. 
Building In-situ Electronic Structure Study Capability with Photon-in/Photon-out

Soft-X-Ray Spectroscopy

Principle Investigator(s): Jinghua Guo

\section{Project Description}

The purposed project is to build in-situ electronic structure study capability of the chemical reactions in photon-in/photon-out soft-X-ray spectroscopy on beamline 7.0 at ALS. The goal is to demonstrate a unique way to handle the real systems (gas-, liquid-phase samples, wet surfaces and interfaces) for electronic structure studies. The focus in science will be on understanding the binding mechanism of hydrogen adsorption and probing efficient hydrogenstorage materials.

The photon-in/photon-out soft-x-ray spectroscopy can be used to study different chemical species of interested in depth, or under the presence of electric and/or magnetic fields, or under wet condition and ambient pressure. Here, the in-situ electronic structure study using photonin/photon-out soft-x-ray spectroscopy will be realized by constructing a gas cell for the electronic structure study of hydrogen adsorption/desorption and a liquid cell for the electronic structure study of liquid mixing and diffusion.

The proposed effort requires the new experimental equipment to be built. The gaseous and liquid phase samples will be maintained in cells inside the vacuum chamber with windows to allow the penetration of soft x-rays. We will build the chemical cells for in-situ photonin/photon-out soft-x-ray spectroscopy studies. The cells include the hydrogen cell for in-situ electronic structure studies of hydrogen adsorption/desorption process involving selected potential hydrogen storage materials and the liquid cell and make it compatible with vacuum environment, and perform a test experiment of in-situ electronic structure studies of molecular liquids and solutions.

\section{Accomplishments}

We have completed an engineering design of the hydrogen reaction cell with an operating temperature range of $80-550 \mathrm{~K}$, and the fabrication effort is ongoing.

It was shown in-situ interaction between SWNTs and $\mathrm{H}_{2}$ gases at room temperature and molecular adsorption caused changes in electronic structure of SWNTs. The raising gas pressure up to 10 torr induces deformation of SWNTs thus decreases the conductance. More molecular gases $\left(\mathrm{CO}, \mathrm{N}_{2}\right.$ and $\mathrm{He}$ ) were tested for understanding the collision that may be responsible to the pressure-dependent spectral change.

We have also been using PVD and chemical solutions to prepare more defined SWNTs, such as metallic or semiconducting tubes without oxidation and defects for hydrogen storage investigation.

A new liquid flow cell for electronic structure study of liquids in a temperature range of 2 $-60{ }^{\circ} \mathrm{C}$ was built, and a number of test experiments of in-situ electronic structure studies of molecular liquids and solutions are performed. 


\section{Soft X-ray Scattering As a New Probe of Polymer Systems}

Alexander Hexemer, Howard Padmore, Zahid Hussain, Jeff Kortright, Nitash Balsara, Rachel Segalman, Ting Xu, Ed Kramer, Sunil Sinha, Harald Ade, Rex Hjelm, Lynn Loo, Thomas Russell

The purpose of this LDRD is to develop Resonant Soft X-ray Scattering (RSoXS). RSoXS is an X-ray probe that can be used to analyze polymers and related hard and soft condensed matter. It combines the conventional hard x-ray scattering with the unique chemical sensitivity of Near Edge X-ray Absorption Fine Structure (NEXAFS) in the soft $\mathrm{x}$-ray regime, which can provide enhanced and tunable contrast between different components. The technique simultaneously provides chemical information, spatial information, and information about interfaces. By using this new technique, researchers will be able to understand how different structures can generate different functional properties in these materials

During FY08, we have obtained access to an experimental scattering chamber at beamline 6.3.1 at the Advanced Light Source (ALS), and have begun to expand its capabilities for RSoXS applications. During the May-June shutdown, we installed an in-vacuum CCD camera, and upgraded and tested a new instrument control system. A special gas cooling setup for the CCD camera was developed and utilized to accommodate the high vacuum requirement for the scattering chamber. New step motors with encoders together with new motor drivers and controllers were installed. This replaced the old servo-motor system for the sample stage control and the control of detector and sample rotation. This system has been specifically tailored to study varies type of samples in reflectivity, transmission and grazing incidence geometries. We have used our limited time on the 6.3.1 chamber primarily to assess its current capabilities and needs in anticipation of ramping up activities next January.

Meanwhile, we have also explored the RSoXS in specular scattering geometry using the existing one dimension detector. One of the projects was to study the effect of interfacial morphology on the performance of multilayer organic light emitting diodes. The interface between active layers in such devices strongly affects performance, however, little is known about those interfaces due to the lack of a suitable experimental technique. Conventional hard xray scattering has very limited sensitivity for the interface of organic thin films due to limited contrast in electron density. Using RSoXS, the multilayer structure of Organic Light Emitting Diodes (OLED) devices was characterized and correlated with the device performance. We observed a correlation between surface roughness and device performance. The OLED's showed better performance for increased roughness at the interface between the electron injection and emission laye. These results will be submitted shortly for publication. 
Carbon-Based Nanostructures as a Prototype for New Electronics

Principle Investigators: Eli Rotenberg and Aaron Bostwick (LBNL);

Steve Kevan (University of Oregon); Karsten Horn (Fritz Haber Institute, Berlin)

\section{Project Description}

The purpose of this project is a spectroscopic investigation of microcrystallites of graphene, a material which has been proposed as the basis for many new classes of devices. Determination of the graphene bandstructure in confined geometries can provide critical spectroscopic information in order to develop such devices, and is possible using the new nanoARPES endstation at the ALS. Aside from the scientific and technological goals, nanoARPES spectroscopy of graphene also serves as a "scientific testbed" for the demonstration of the nanoARPES technique which is evolving in parallel with this project. In this LDRD, we proposed to create, locate, and image graphene crystallites.

\section{Accomplishments}

Our principle accomplishment was to successfully measure the structure and basic electronic properties of fixed and freestanding graphene samples (see figure below) ${ }^{\dagger}$. These images are the first microscopic pictures of graphene obtained using valence band electrons (for graphene on $\mathrm{SiO}_{2}$ ) or chemically-resolved core level (for freestanding graphene) as contrast mechanisms. For graphene on silicon oxide substrates, we could determine not only the sample thickness (number of graphene layers) but also simultaneously probe the valence and core level electronic spectra providing fundamental information on the chemical state and electronic properties (data not shown).

(a) OPTICAL MICROGRAPHS

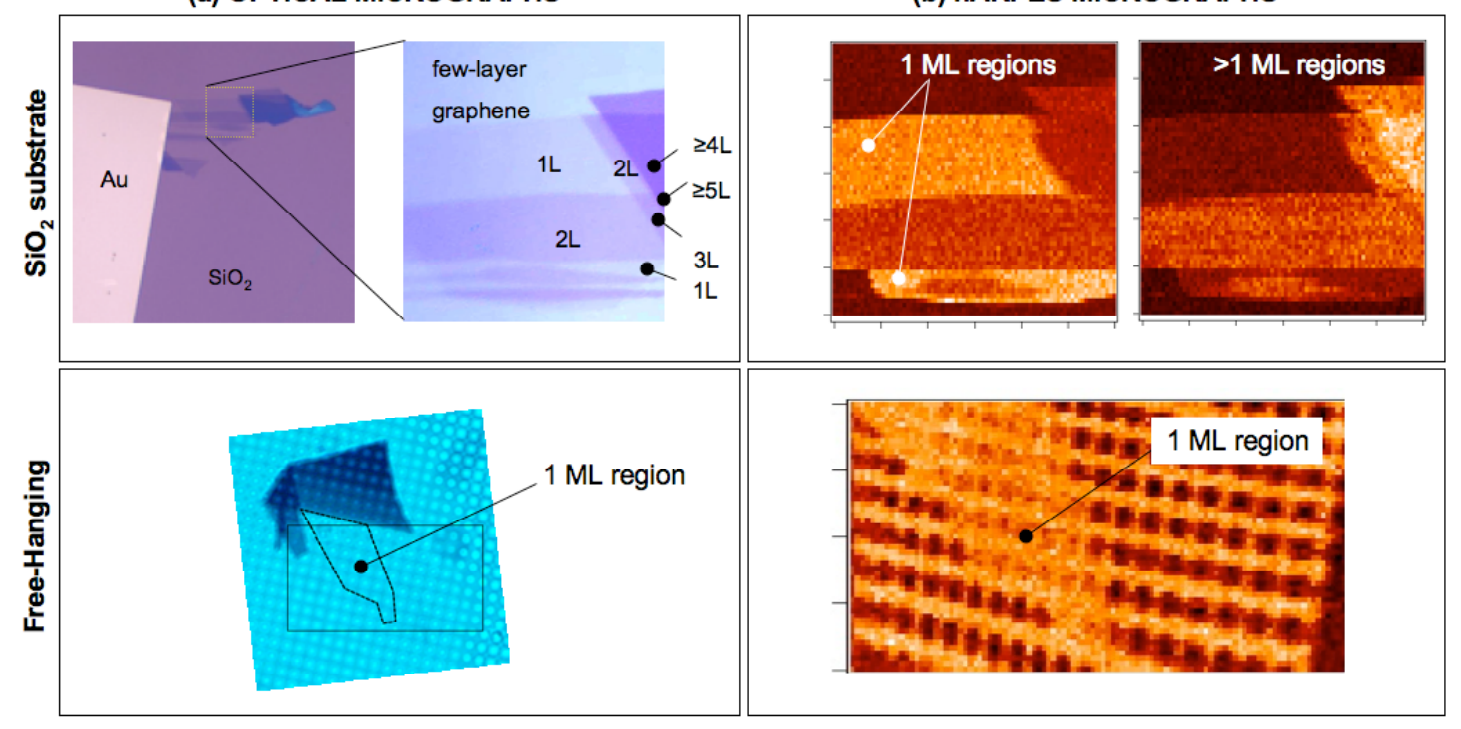

Figure. (a) optical and (b) nanoARPES images of exfoliated (upper row) graphene on $\mathrm{SiO}_{2}$ substrate and (lower row) carbon mesh. The graphene flake is barely visible in the optical micrograph and its presence is indicated by the dotted line. The nARPES micrograph clearly shows the monolayer graphene sample. The holes in the carbon mesh constitute regions of freely suspended graphene. 
Development of a laser goniometer for soft x-ray diffraction microscopy Principal Investigators: David Shapiro and Yiqiong Zhao

Project Description:

Coherent x-ray diffraction microscopy is a lensless microscopy that can produce high resolution images of materials from only a measurement of their far-field x-ray diffraction pattern. In principle, the lack of lenses means that the achievable resolution is limited only by the x-ray wavelength and the scattering angle to which adequate photons are counted. The x-ray diffraction phase problem is solved through adequate sampling of the diffraction pattern. Reliable sampling of the pattern requires that the coherence length of the illuminating radiation be at least twice as big as the sample of interest and that the sample be surrounded by a zero scattering region. In practice, this is very difficult to achieve and more often than not, parasitic scatter from the specimen's support membrane contributes significantly to the recorded diffraction pattern. This parasitic scatter can have different effects depending on its nature but in general it will make the phase problem more difficult to solve resulting in lower resolution images and a less reliable imaging method.

The goal of this project is to develop a laser trap ("laser tweezers") that can levitate, translate, and rotationally orient micron sized particles in a gaseous environment so that they may be used in $\mathrm{x}$-ray diffraction microscopy experiments without a support membrane. This will result in a total reduction of parasitic scatter from the diffraction patterns and result in a more reliable and higher resolution imaging system. It will provide the possibility of few-nm resolution imaging, in three dimensions, of several-micron sized material sciences samples. In the biological sciences, it offers the opportunity to combine established optical techniques, such as fluorescence microscopy and Raman spectroscopy, with high resolution x-ray imaging. We will use a standard optical tweezers design that is modified to allow delivery of aerosolized particles to the trap and we will develop a means of dynamically adjusting the illumination to allow controlled rotations of the trapped particles.

Accomplishments

We have implemented a laser trap that is capable of optically trapping particles in solution and particles in air that have been delivered by a nebulizer. The trapping potential is created by passing a high-power, $1064 \mathrm{~nm}$ wavelength laser through a high-numerical aperture microscope objective and the diffraction samples are delivered by a nebulizer (containing the particles in solution) in the vicinity of the trap. The optical trap also allows passage of white light illumination for standard imaging, and $635 \mathrm{~nm}$ wavelength laser light for scattering measurements. Motorized manipulation of relay mirrors along the trapping beam path allows for controlled translation of the trapping potential (and hence the trapped particle) in three dimensions. We have optimized the trapping power for particles of 2-3 micron diameter which is the optimum size for x-ray diffraction microscopy measurements.

We are currently in the process of designing the apparatus that will mount the laser trap within a coherent soft x-ray beamline at the ALS (BL 9.0.1) and we are developing the means of particle rotation. This involves breaking the cylindrical symmetry of the trap by introducing a rotatable slot aperture into the trapping laser's beam-path. 
Ultra-high resolution optics for soft x-ray inelastic scattering

Principal Investigator(s): Valeriy V. Yashchuk

\section{Project Description}

The goal of this work is to demonstrate and establish new technology required for ultrahigh resolution Resonant Inelastic soft X-ray Scattering (RIXS). RIXS is one of the most important potential new probes in electronic structure determination due to its elemental selectivity, high momentum resolution, and ability to probe bulk materials. However, important advantages of the method are limited due to energy resolution limits imposed by traditional grating optics, especially at soft x-ray energies. For example, energy scales imposed by collective phenomena in correlated electronic systems dictate that meV energy resolution is required for experiments at 500-1000 eV energy, requiring a resolving power of about $10,{ }^{6}$ unachievable with conventional optics.

We have proposed and currently work to realize a revolutionary new way to construct gratings with ultra-high groove density, potentially up to $50,000 \mathrm{~mm}^{-1}$ to get to the high resolving powers required. We fabricate such a grating by deposition of a soft xray multilayer on a substrate which is a blazed saw-tooth grating (echellette) with low groove density. Subsequent polishing applied to the coated grating removes part of the coating and forms an oblique-cut multi-line structure that is a sliced multilayer grating. The resulting grating has a short-scale periodicity of lines (bilayers), which is defined by the multilayer period and the oblique-cut angle.

\section{Accomplishments}

Our most significant accomplishment has been to fabricate and characterize a $\mathrm{Sc} / \mathrm{Si}$ multilayer sliced grating suitable for EUV applications, which is a first realization of the suggested technique.

In order to fabricate an echellette substrate, we used anisotropic $\mathrm{KOH}$ etching of a Si wafer. The etching regime was optimized to obtain smooth and flat echellette facets. A $\mathrm{Sc} / \mathrm{Si}$ multilayer was deposited by dc-magnetron sputtering, and after that it was mechanically polished using a number of diamond pastes. With groove density of $\sim 3700$ lines per $\mathrm{mm}$, the grating prototype is suitable for EUV applications. Although the groove density is smaller than that required for ultra-high resolution spectroscopy with soft $\mathrm{x}$ rays, the prototype grating is rather convenient for characterization with a number of techniques, including scanning probe, electron, and interferometric microscopy. This allows checking the consistency of the approaches proposed, as well as optimizing the grating fabrication technology.

We have tested the resulting $\mathrm{Sc} / \mathrm{Si}$ multilayer sliced grating prototype in diffraction experiments with the ALS BL6.3.2 spectrometer. The performed measurements have provided a lower limit of $\sim 400$ for the resolution of the sliced grating at a diffraction efficiency of $\sim 7 \%$ for the optimized 38th order assigned to the echellette grating of $10 \mu \mathrm{m}$ period. Because the resolution limit is due to the finite resolution of the BL6.3.2 spectrometer, the experimental results are a proof-of-principle demonstration that a sliced diffraction grating fabricated as suggested possesses an expected high efficiency of diffraction in the EUV (41-49 nm photon wavelength) range.

We are in the process of extending the developed techniques to fabricate ultra high density gratings for soft x-rays. We will also design an experimental procedure and set-up for characterization of the diffractive properties of the high density gratings. 


\section{Chemical Sciences Division}

LB07003

Synthetic and Electrochemical Approaches to Metal-Metal Bonds in Actinides Principle Investigator: Dr. John Arnold

\section{Project Description}

The isolation of complexes containing actinide metal-metal bonds remains an illusive goal. The recent emergence of computational studies suggesting that species such as $\mathrm{U}_{2}, \mathrm{U}_{2}{ }^{2+}$ and $\mathrm{Th}_{2} \mathrm{H}_{2}$ for example should be stable, and that $5 f$ orbitals are key components in the bonding of such species, remain in practical terms as tantalizing suggestions. We have a long-standing interest in using ligand design to influence and promote unusual structural motifs, and we believe that we can apply our chemistry to this important and chemically fascinating subject. Through design and application of new ligand systems, we intend to influence the structure of generated actinide complexes such that metal-metal bond formation with actinides is rendered energetically feasible.

\section{Accomplishments}

Our initial efforts to synthesize molecular actinide compounds with metal-metal bonds focused on manipulation of coordinative unsaturation. In this method, a suitable actinide starting material is selected with a "pocket" for the other unsaturated metal starting material to coordinate. Typically actinide complexes adopt the maximum possible coordination number by coordinating solvent molecules. The soluble triscyclopentadienyl uranium(III) complex $(\mathrm{CpSiMe})_{3} \mathrm{U}$ was chosen because it can be prepared in the absence of coordinating solvents. Considering the propensity for the U(III) metallocenes to bind soft $\pi$-accepting ligands, we began exploring the reactivity of these complexes toward the group 13 diyls $\mathrm{Cp}^{*} \mathrm{M}$. The $\left(\mathrm{Cp}^{*} \mathrm{Al}\right)_{4}$ tetramer dissociates at elevated temperature into $\mathrm{Cp} * \mathrm{Al}$ monomers, and $\mathrm{Cp} * \mathrm{Ga}$ is monomeric at room-temperature. Each diyl possesses a pair of electrons on the metal atom allowing it to act as a Lewis base, and are formally isolobal with singlet carbene, $\mathrm{CO}$, and $\mathrm{PR}_{3}$.

Reactions of $(\mathrm{CpSiMe})_{3} \mathrm{U}$ with $\mathrm{Cp} * \mathrm{M}(\mathrm{M}=\mathrm{Al}, \mathrm{Ga})$ resulted in crystals of $(\mathrm{CpSiMe})_{3} \mathrm{U}-$ MCp*, following crystallization from pentane in moderate yields. Both complexes have been fully characterized by standard analytical techniques including single-crystal X-ray diffraction analysis, ${ }^{1} \mathrm{H}$ and ${ }^{13} \mathrm{C}$ NMR, UV/VIS/NIR spectroscopy, and elemental analysis. The possibility of bridging hydride ligands was ruled out through careful scrutiny of the X-ray crystal structures, mass-spectrometry, IR and NMR spectroscopy, and in reactivity studies.

Though alloys of uranium with aluminum and gallium are known, these are the first examples of molecular actinide-group 13 bonds. The U-M bonds (U-Al = $3.12 \AA$; $\mathrm{U}-\mathrm{Ga}=3.07$ $\AA$ ) are slightly shorter than would be predicted by the sum of their covalent radii ( $\mathrm{U}-\mathrm{Al}=3.17$ $\AA, \mathrm{U}-\mathrm{Ga}=3.18 \AA$ ). Preliminary results from an ongoing DFT study suggest that there is a degree of covalent character to the U-Al bond. A natural bond orbital (NBO) analysis yielded natural charges of 1.899 and 0.594 for $\mathrm{U}$ and $\mathrm{Al}$, respectively. A natural charge of 0.540 was computed for the AlCp fragment, indicating that there is a net $\mathrm{Al} \rightarrow \mathrm{U}$ charge transfer, albeit small. However, the Wiberg bond index between $\mathrm{U}$ and $\mathrm{Al}$ is 0.487 , indicating the presence of a covalent bond of order ca. 0.5 . 
Conversion of Glycerol, other Carbohydrates and Aromatic Compounds from Biomass to Major 3- and 6- Carbon Industrial Organic Compounds Principal Investigators: Robert G. Bergman and Jonathan A. Ellman

November 20, 2008

\section{Project Description}

Biomass-derived intermediates are potential sustainable substitutes for petroleumbased building blocks in the production of commodity chemicals. In this context, the transformation of biomass materials into small, carbon-rich compounds is an important challenge. Glycerol is the main byproduct in the triglyceride transesterification process for biodiesel manufacture. The market expansion of biomass-derived fuels has resulted in an increase in glycerol production with a subsequent drop in price, rendering it extremely attractive as a starting material for the synthesis of more valuable chemicals.

\section{Accomplishments}

We found and optimized a selective one-pot, formic acid-mediated deoxygenation technique for converting glycerol to allyl alcohol. Our mechanistic studies using labeling experiments revealed an unexpected concerted mechanism for this transformation, involving a carbocation cyclic intermediate, and this have led us to devise a general diol deoxygenation reaction.

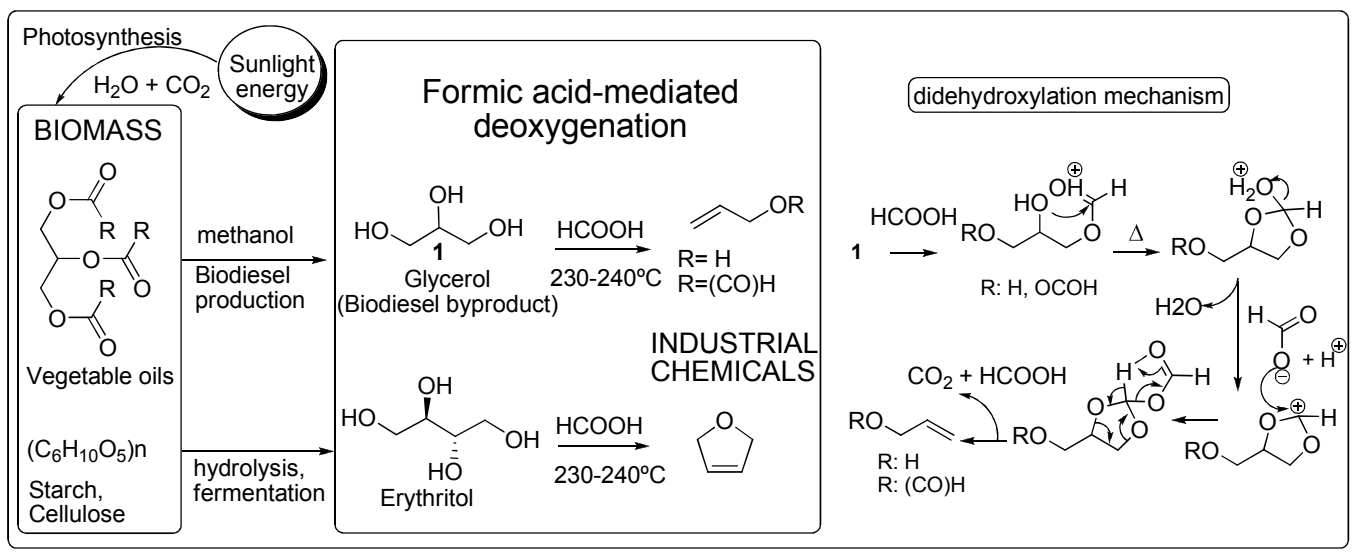

The reaction was found applicable in the case of 1,2-diols such as 1,2-octanediol, cyclic diols such as cis-1,2-cyclooctanediol and trihydroxy compounds such as 1,2,3hexanetriol that afforded the corresponding olefins in high yields. Deoxygenation of diols to produce olefinic compounds is a useful synthetic transformation. The formic acid-mediated didehydroxylation provides a one-step and simple procedure that directly affords the alkenes in high yield and purity.

One application of this process has generated a further biomass transformation: the first example of formic acid mediated cyclization/deoxygenation of erythritol, a four-carbon sugar polyol. 2,5-Dihydrofurans are important building blocks for the pharmaceutical and commodity chemical industry. The transformation of erythritol to 2,5-dihydrofuran is a good example of how this reaction could offer an efficient approach to the conversion of biomass to value-added chemicals. This mild, one-step and inexpensive procedure is a promising tool for the development of biomass refinery processes. 


\title{
Chemical reactions at liquid/vapor interfaces probed by photoemission spectroscopy
}

\author{
Principal Investigators: Hendrik Bluhm and Kevin R. Wilson
}

\section{Project Description}

The goal of this project is to build a novel instrument for the investigation of chemical reactions at liquid/vapor interfaces under atmospherically and environmentally relevant conditions. The properties of liquid/vapor interfaces strongly influence heterogeneous processes in atmospheric and environmental chemistry. In particular aqueous aerosols catalyze many heterogeneous reactions in the troposphere and function as both sinks and sources for atmospheric trace gases. Up to now little is known about the concentration of solution phase species at the liquid/vapor interface, which can significantly differ from the bulk solution concentration and is an important quantity in the modeling of heterogeneous reactions at liquid/vapor interfaces. A direct measurement of the liquid/vapor interface under atmospherically and environmentally relevant conditions is difficult due to the lack of quantitative experimental techniques that are surface-sensitive, chemically specific and can operate at elevated pressures in the Torr range. By combining synchrotron-based ambient pressure photoemission spectroscopy (APPES) and a liquid droplet train into a single experimental setup we will address this need and provide quantitative information about the chemical nature of the liquid/vapor interface under varying conditions of bulk concentration and temperature, as well as gas phase composition and pressure.

We have planned our experiments in two stages. In the first phase we have implemented a droplet train generator in the ambient pressure photoemission endstation at beamline 11.0.2 at the Advanced Light Source. This setup allowed us to optimize the experimental parameters and, in addition, to perform first experiments on solution/vapor interfaces. We are currently in the second phase where we are building a dedicated machine that will allow us to vary the interaction time between droplets and gas molecules. This will enable us to investigate the kinetics of heterogeneous reactions that have great relevance to atmospheric chemistry but are currently incompatible with conventional ultra-high vacuum systems (e.g., $\mathrm{HNO}_{3}, \mathrm{H}_{2} \mathrm{SO}_{4}$, and $\mathrm{HCl})$.

\section{Accomplishments}

In the first stage of this project we have successfully implemented a droplet train generator in the APPES endstation at beamline 11.0.2. The train of droplets is positioned close to the entrance aperture of the electrostatic lens system of the APPES endstation where incident photons from beamline 11.0.2 irradiate the droplets in front of the aperture, and the resulting electrons are collected by the differentially pumped lens system and probed using the hemispherical analyzer. To test the feasibility of our approach we have conducted a number of proof-of-concept experiments on various liquid/vapor interfaces, including aqueous salt and alcohol solutions, which resulted in the publication of a paper in Phys. Chem. Chem. Phys.

During the last year we have worked on the setup of a new, dedicated droplet train/APPES instrument. We have developed a new flow tube design that allows for the variation of the exposure of the droplets to trace gases without changes of the trajectories of the droplets, a crucial prerequisite for successful APPES experiments on droplets. A feedback mechanism for the control of the droplet trajectories has been built. In addition, a new APPES spectrometer with increased efficiency has been developed and will be operational at the end of March 2009 at beamline 11.0.2. 


\section{Probing Transient Molecular Entanglement using \\ Femtosecond High Resolution Delayed-Field Coincidence Imaging \\ Principle Investigator(s): Oliver Gessner}

\section{Project Description}

This project is aimed to study the transient entanglement of the constituents of a molecule during unimolecular dissociation in real-time. It will explore the very heart of chemical bonding: the boundaries and the transition between separate particle and unified molecular behavior. The quantum mechanical nature of chemical bonding over potentially macroscopic distances and the conditions to create entangled particles through unimolecular dissociation in the gas phase will be investigated.

A newly designed high resolution delayed-field photoelectron-ion coincidence imaging spectrometer will be used in combination with the high repetition rate ultrafast soft X-ray source of the Ultrafast X-ray Science Laboratory (UXSL) at LBNL. The studies will constitute a significant contribution to the UXSL endeavor in becoming one of the world's leading centers for ultrafast X-ray science. They will aid in paving the way for future science performed at ultrafast X-ray light sources like the Stanford LCLS, and help to define benchmark requirements for a new FEL-based ultrafast X-ray source at LBNL.

Accomplishments

Within the first 5 months of funding an international search for a post-doctoral fellow has been successfully completed with the hire of Andrew Healy. A recipient of the University of Minnesota Overend Award for Graduate Research in Physical Chemistry, he started his position in February 2008. Major components for the experimental apparatus such as turbo- and backing pumps, a piezo-driven high repetition rate pulsed valve and a time-and position-sensitive ion detector have been acquired.

Vital to the experiment is the availability of well characterized femtosecond duration light pulses in the EUV. An ultracompact EUV spectrometer and beam profiler has been designed and constructed and is now operated on a routine basis to characterize the spectrum and spatial mode of femtosecond EUV pulses created by high-order harmonic generation from a $785 \mathrm{~nm}$ fundamental light pulse. The spectrometer has also enabled us to characterize, for the first time, the photon-energy dependent reflectivity of multilayer optics provided by the LBNL Center for X-ray Optics with ultrashort EUV pulses and in the photon energy regime relevant to our experiment. LBNL has filed a provisional patent application for this invention.

A collaboration with the Leone group at LBNL and UCB has been established which allows us to perform first test experiments on existing apparatus while our dedicated experimental setup is still under construction. First experiments on the transient entanglement in the unimolecular dissociation of $\mathrm{Br}_{2}$ are under way. 


\section{Metal Nitrosyl Complexes and Catalytic C-H Bond Functionalization \\ Principal Investigator(s): F. Dean Toste}

\section{Project Description}

The direct functionalization of carbon-hydrogen bonds to form reactive carbonmetal that can undergo further transformations has been the focus of intense effort. While significant advances have been made in the activation of aromatic $\mathrm{C}-\mathrm{H}$ bonds, in stark contrast, considerably less progress has been made towards the selective activation of olefinic C-H bonds. Given that alpha-olefins are a common chemical feedstock and important intermediates in fine chemical synthesis, the goal of this proposal is two-fold: (1) develop a new concept for the catalytic activation of $\mathrm{C}-\mathrm{H}$ bonds of unfunctionalized alkenes using the ligand-based reactivity of metal dinitrosyl complexes with the substrate undergoing $\mathrm{C}-\mathrm{H}$ functionalization (2) apply this concept to the catalytic functionalization of olefinic $\mathrm{C}-\mathrm{H}$ bonds.

Our approach relies on rational organic and organometallic synthesis to propose and develop a catalytic cycle for the functionalization on olefinic $\mathrm{C}-\mathrm{H}$ bonds. In this endeavor we will first study and develop the individual steps in the catalytic cycle. Understanding the underlying reactivity of the individuals steps will not only allow to optimize the ultimate catalytic process, but also to gain insight into novel organic and organometallic reaction mechanisms and fundamental processes. Therefore, we hope that this approach will not only directly impact our goal, but also inspire and influence other catalytic reactions.

\section{Accomplishments}

To date, we have accomplished all of the stoichiometric reactions required for the proposed catalysis. The sequence shown below for the $\mathrm{C}-\mathrm{H}$ functionalization of cyclopentene is illustrative. We initially prepared of a variety of stable, isolable cobalt dinitrosoalkane complexes by treating of the cobalt dicarbonyl complex with an excess of alkene in the presence of NO. For example, cyclopentene derived cycloadduct 1 was formed in $92 \%$ yield by this method. Treatment of dinitrosoalane complex with base presumably generates an anion that can be reacted with electrophiles. We have found that electron deficient olefins (i.e. Michael acceptors) react with the deprotonated dinitrosoalkane in the presence of lanthanide-based Lewis acids and HMPA as a cosolvent. Finally, thermal retro-cycloaddition releases the functionalized alkene. For example, cyclopentene 3 was formed in $92 \%$ yield by heating of 2 at $75^{\circ} \mathrm{C}$.

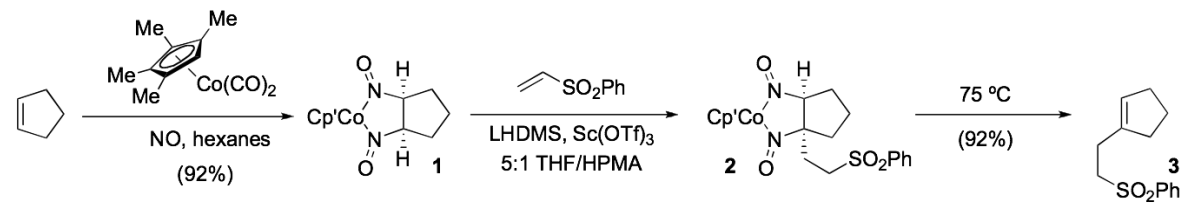




\section{Computing Sciences}

On-Demand Overlays for Scientific Applications

Principal Investigator(s): Deb Agarwal, Chin Guok

\section{Project Description}

Scientific computations and collaborations increasingly rely on the network to provide high-speed data transfer, disseminate results, access instruments, support computational steering, etc. Networks with redundant high-speed paths need algorithms to effectively allocate bandwidth across multiple paths for a single application. This capability is required for two important communication classes within the DOE scientific community: large point-to-point transfers and periodic data dissemination from a single sender to multiple receivers. The overall goal of this project is to perform the research and development necessary to create proof-of-concept on-demand overlays for scientific applications that make efficient and effective use of the available network resources

Application overlay networks have been effective in providing data aggregation and dissemination services in the general Internet. An application overlay network creates a virtual application-layer topology that sits on top of the physical network. This application-layer topology can be dynamically configured either through a centralized decision point or a distributed algorithm. This enables the application overlays to optimize network utilization for the application data traffic. This is in contrast to current Shortest Path First (SPF) approaches that always choose a single "best" path. The majority of overlay algorithms and protocols have been designed to work at the application layer of the networking stack. We believe that the existing work at the application layer can be applied to produce algorithms and protocols that make efficient and effective use of resources at lower layers of the network stack.

\section{Accomplishments}

In this project we have focused on developing mechanisms for instantiating ondemand virtual overlays on the physical ESnet network and integrating those mechanisms with the OSCARS dynamic circuit reservation system. This past year, we developed a prototype system to transparently route user traffic onto an overlay network on ESnet using the Phoebus system from the University of Delaware. This new routing mechanism allows users to take full advantage of the on-demand overlay network without any changes to their existing applications. We also conducted a range of tests to understand the effectiveness of the Phoebus overlay network for bulk data transfer applications. Both HTTP and GridFTP were used as the bulk transfer application. Our tests show the value of the overlay network, especially when integrated with the OSCARS dynamic circuit network (DCN). One important result from these tests was an understanding that using an overlay network allows for protocol translation between nodes in the overlay. Significant performance gains were seen by using TCP at the edges and UDT over the DCN links. Our work has resulted in two proposals that were recently submitted to DOE, and we are currently preparing a paper describing the research undertaken in this project. This paper, in addition to describing the research, will summarize our testing results. 
A Structured, Adaptive Mesh Refinement Method for Multiphase Reactive Transport in Groundwater

Principle Investigator: John B. Bell

\section{Project Description}

High-fidelity simulations of groundwater flow have the potential for providing valuable insights into long-term fate of contaminants. However, realizing this potential presents significant computational challenges. We propose to perform a proof of concept study to demonstrate the effectiveness of adaptive mesh refinement (AMR) for reactive subsurface transport. The project will develop a basic flow module for modeling multiphase, multicomponent non-isothermal flow with reactions. This methodology will include second-order discretization methods that provide a robust and accurate treatment of transport processes and the coupling of that integration scheme to parallel AMR methodology. The overall goal is to validate and demonstrate the effectiveness of this approach.

The flow of subsurface fluids is governed by conservation laws for chemical species and energy, including the effects of geochemistry and phase behavior, together with phase velocities given by Darcy's law. Our approach is based on a sequential formulation of the porous media flow equations. In this approach we first solve a pressure equation. We then solve the multicomponent conservation and energy equations using a fractional flow formulation. The overall integration approach is combined with AMR methods that include support for large distributed multiprocessors. Specialized linear solver must also be developed to address the anisotropic meshes typically used for subsurface flow problems.

\section{Accomplishments}

We have made two significant accomplishments in this project this year. First, we have extended the AMR framework for subsurface flow we developed last year to three dimensions. Our approach is second-order in space and time and supports subcycling in time so that finer spatial grids are advanced with smaller time steps. Specialized synchronization algorithms are used so that the integration of the finer levels of refinement does not require global elliptic solves over the entire grid hierarchy. The method has been parallelized and shows excellent weak scaling behavior up to several hundred processors. We have also extended the method to treat multiphase systems and have validated that capability on a three-component, two-phase model.

The other major accomplishment has been the incorporation of realistic geochemistry into the algorithm. The overall discretization strategy is based on a secondorder operator split approach that allows us to use stiff ordinary differential equation methodology to integrate the chemical reactions. We are using an interface to the geochemistry package used by TOUGHREACT to provide kinetic rate evaluation. As part of this extension we also have to add the capability to model molecular diffusion to the AMR framework. We have tested the geochemistry model on a number of problems including a seven component system that forms a plume of calcite precipitate. 
Low Order Models for Simulation of Ballistic Charge Transport in Nanoscale Devices Principle Investigator: John B. Bell

\section{Project Description}

As size of electronic devices shrinks to nanometer scale, ballistic charge transport becomes increasingly important in describing the transport phenomena in these devices. However, its simulation is usually computationally intensive due to the large number of states required to accurately determine the distribution of the electron density and the number of self-consistent iterations needed to achieve convergence. The project will develop a computational strategy that utilizes model-order reduction techniques. The goal is to validate and demonstrate the effectiveness of the resulting approach.

Our formulation for the ballistic charge transport is based on the Schröedinger Poisson system of equations that must be solved self-consistently. Our proposed approach integrates the reduced basis method, a model-order reduction technique which exploits dimension reduction afforded by the smooth and low-dimensional parametrically induced solution manifold, with the subband decomposition method. We also exploit $a$ posteriori error estimation procedure to systematically control the accuracy of our solutions. We will initially examine its application to a double-gate nano-MOSFET device. We anticipate its greater utility in the rapid design of nanotubes-based devices.

\section{Accomplishments}

We have made two significant accomplishments. First, we formulated a consistent weak formulation for the mode-space approach that allows clear parametric decomposition. It allows us to identify model reduction opportunities that we can exploit. We have also formulated good asymptotic error bounds that will function as error estimators for our reduced basis approximation.

Second, we have demonstrated that our approach is indeed very efficient for the double-gate nano-MOSFET model problem. Numerical results show that the computational cost is reduced by $50 \%$ for a reasonably sized problem and depends very weakly on the mesh size in the confined direction. We expect the computational savings to increase in cases of two-dimensional confinement, such as those encountered in nanowires and nanotubes.

We are in the process of developing the computational machinery necessary to perform ballistic transport simulation in three-dimensional devices. We are also interested in identifying model reduction opportunities in the related time-dependent problem. 
Enhancing the Effectiveness of Manycore Chip Technologies for High-End Computing Principal Investigators: Jonathan Carter, Leonid Oliker, and John Shalf

Project Description

The goal of this project is to investigate the key challenge for next-generation highend computing systems - effectively utilizing computing systems with exponentially increasing numbers of cores per chip. Recent evidence points to the fact that future petascale computing platforms will be constructed from chips containing an increasing number of cores. It also points to a convergence towards manycore designs that may include hundreds or even thousands of cores.

Our work examines a broad range of manycore systems from small loosely-connected systems to tightly-coupled supercomputers, exploring both heterogeneous and homogeneous designs. We have started to evaluate single-socket manycore architectures across a key set of algorithms using various application probes. Additionally, we have conducted studies on large-scale manycore systems, to explore the effect of these technologies on candidate petascale DOE applications. Further, since power is becoming much more of an issue with deploying high-end computers, we will evaluate power consumption for these technologies where possible.

These advances can be transferred to the broader scientific community to improve the efficiency of simulations. In addition, our results will provide a basis to engage and influence system designers and the computational community. Finally, our work will contribute to LBNL's role of defining and fielding the most effective computing technology for large scale scientific computing.

Accomplishments

A large fraction of scientific applications in such diverse areas as heat diffusion, electromagnetics, and fluid dynamics are formulated in terms of partial differential equations. These applications often use iterative finite-difference techniques, which sweep over a spatial grid, performing nearest neighbor computations called stencils. We have used a microbenchmark, which encapsulates the essential features of stencil computations, and ported and optimized it for a wide range of manycore architectures. Optimizations include cooperative multithreading, where multiple threads work on the same cache line, and register and cache blocking. The results from this study have been presented at the Supercomputing '08 conference.

Lattice-Boltzmann models offer an alternative to direct numerical simulation techniques, and have been used extensively in the area of fluid flows in complex boundaries, reactive flows, porous media, and more recently in magnetohydrodynamics (MHD). Similar to the stencil probe above, our study has shown considerable improvements in performance for a wide variety of architectures. Currently, we are working on extending the architectures covered, and on producing a large-scale parallel version.

Particle-in-cell (PIC) techniques are widely used to simulate physical phenomena, including the emergence of turbulence in nuclear fusion experiments. Using the PIC application Gyrokinetic Turbulence Code (GTC), we have identified the most computationally expensive pieces of this technique and have started to optimize this kernel and obtain comparative performance for a range of manycore architectures. 


\title{
Integrated Microbial Community Genomes Data Management System
}

\author{
Principal Investigators: Victor M. Markowitz, Natalia N. Ivanova, \\ Nikos C. Kyrpides, and Phil Hugenholtz
}

\section{Project Description}

Studies of the collective genomes (also known as metagenomes) of environmental microbial communities (also known as microbiomes) are expected to lead to advances in environmental cleanup, agriculture, industrial processes, and alternative energy production. Similarly, studies of the metagenomes of human microbiomes will provide new insights into variations of microbial populations associated with the human body in health and disease, and will lead to the development of new treatment strategies.

The purpose of this project is to develop metagenome data management and analysis tools in the context of integrated microbial genome and metagenome data. These tools will provide support for the integration and interpretation of metagenome sequence data and sample metadata; functional annotation and metabolic reconstruction for metagenome sequence data; comparative data analysis and visualization of metagenome sequence data in the context of sample metadata and isolate microbial genome sequence data. Metagenome data analysis tools These tools will allow addressing fundamental biological questions, such as how are functions distributed within a community and how does a community respond in both the near and long term to environmental changes.

\section{Accomplishments}

In FY 2008, additional metagenome data management and analysis tools were developed and provided to scientists for analyzing environmental and human microbiome metagenomes. The content of IMG/M was updated twice during FY2008, and includes (as of September 2008) a total of 3,640 genomes consisting of 819 bacterial, 50 archaeal, 40 eukaryotic genomes, and 2,044 viruses, integrated with metagenome sequences generated from 46 microbial community samples. A new "Expert Review" version of IMG/M was set up to support metagenome studies towards their publication: IMG/M ER contains 123 metagenome datasets that are analyzed as part of 40 ongoing studies.

Data processing for handling metagenome sequences generated using 454 Life Sciences pyrosequencing technology have been gradually extended and improved. Over 30 such datasets have been processed and then included into IMG/M ER. Additional metagenome data analysis tools have been developed to support the exploration and comparative analysis of metagenomes in the context of isolate microbial genomes and other metagenomes. Tools for comparing the functional capabilities of metagenomes have been extended with tests for estimating the statistical significance of the observed differences.

IMG/M has proved valuable in completing the analysis of the recently published studies on a microbial community from Lake Washington in Seattle (Nature Biotechnology, August 2008) and a hypersaline microbial mat (Molecular System Biology 4, June 2008).

A recently awarded NIH grant will allow continuing work on IMG/M as part a new Data Analysis Coordination Center (DACC) for the Human Microbiome Project (http://nihroadmap.nih.gov/hmp), with the goal of expanding IMG/M's capabilities to support the analysis of human metagenome data. 


\title{
Energy Smart Disk-Based Mass Storage System
}

\author{
Principal Investigator(s): Doron Rotem, Ekow Otoo
}

\section{Project Description}

The Energy Smart Disk-Based Mass Storage System project is intended to address system software approach for implementing energy efficient storage systems. The primary goal is to investigate, implement and deploy modules for the management of reliable low-cost disk-based storage systems that maximize the energy savings while meeting the performance goals of the storage systems with respect to access latency, reliability and operational costs. The main ideas involve: i) utilizing the concepts of a massive array of idle disks (MAID) where idle disks are powered down when not in use; ii) consolidating resource use in both time and space to facilitate powering down unused hardware components; and iii) implementing energy aware cache replacement policies and algorithms when emerging hybrid-disks technology, i.e., disks augmented with flash memory, are deployed. The project's goals also include the development of analytical and experimental simulation models for analyzing energy consumption of different configurations of these system and configuring a prototype system the embeds our solutions. For example one specific problem addressed is as follows: "Given a workload of accesses for $n$ files, and with a specified maximum bound on the response time, what is the minimum number of disks (of some specified characteristics) needed?"

\section{Accomplishments}

Our main accomplishments to date include first the development and implementation of a file allocation algorithm on disks to achieve maximal power conservation while satisfying the response time constraints. This was achieved by mapping the problem to a generalized bin packing problem called two-dimensional vector packing problem (2DVPP). This mapping allowed us to use algorithms that solve 2DVPP with provable bounds from the optimum. More specifically, we developed two algorithms that improves on the running time of a 2DVPP algorithm (that has the best known bounds from optimality), from $O\left(n^{2}\right)$ to one of $O(n \log n)$ and a second of $O(n)$, where $n$ is the number of files allocated. The second accomplishment achieved include the use of extensive simulations with both simulated and realistic workloads and accurate disk characteristics, to calculate the energy savings and response times achieved by using our file allocation techniques as compared with random file allocation. We demonstrate that our techniques achieve significant energy savings over a wide range of workload parameters values with minimal response time degradation. Figures 1 and 2 depicts respectively the relationships between power savings and response time for different file access rates.
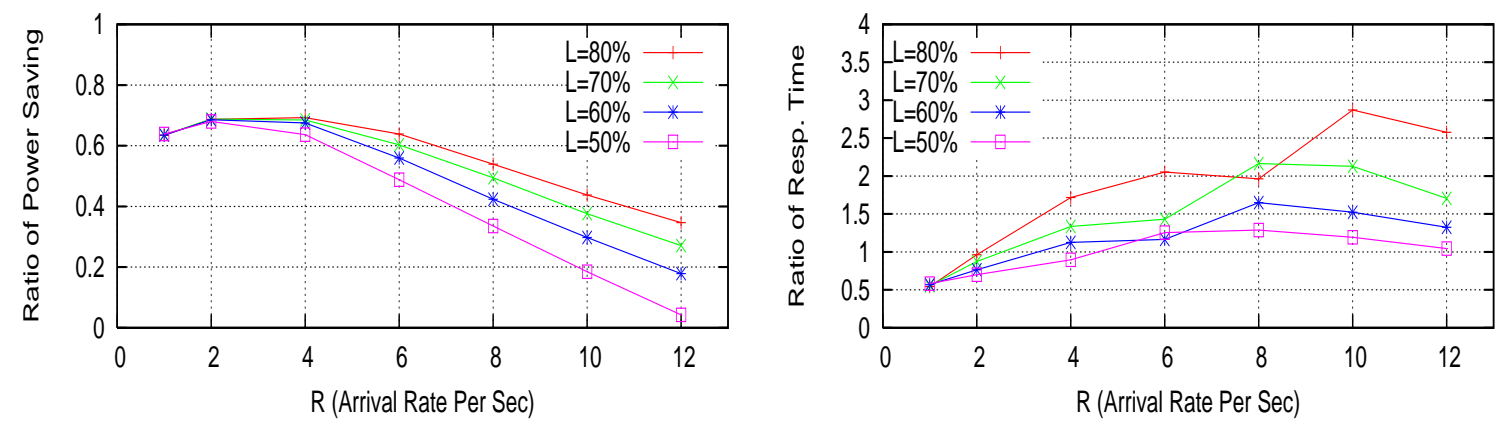

Figure 1: The ratio of power saving of Pack_Disks Figure 2: The ratio of response time of algorithm to random allocation at different arrival Pack_Disks algorithm to response time of random rates of file access allocation 


\title{
A Holistic Approach to Energy Efficient Computing Architecture
}

\author{
M. Wehner, L. Oliker, J. Shalf
}

\section{Project Description}

The goal of this project is to develop new technology for energy-efficient computational science. The approach is to take a vertical slice through the space of applications, algorithms, software, and hardware to perform a study on how to build the most energy-efficient system to solve a particular computation science problem. The initial target will be climate simulation, and the solution is likely to require higher degrees of parallelism in the climate algorithms and software, as well as new hardware designs based on technology from outside the traditional workstation and server domains, particularly low-power embedded technology. The result will be a proof-of-concept machine design, along with supporting analyses of how this compares to alternative designs, and new algorithms and software techniques as needed to make the case for feasibility of computing the science problem on the system. . The project is led by John Shalf and will coordinate with other energy-efficient projects, including the LDRDs by Erich Strohmaier, Jonathan Carter, and Doron Rotem.

\section{Major Milestones in 2008}

We have demonstrated a full application based on Celal Konor's limited area icosahedral climate code model, using a prototype of a processor building-block of a power-efficient processor design using a Berkeley Emulation Engine-3 hardware simulation environment at the SC2008 conference in November of 2008 in Austin, Texas. This demonstration resulted from substantial progress in the areas described below.

\section{Code Analysis}

Ongoing effort toward the analysis of the Icosahedral limited area atmospheric hydrodynamic model v74, AHM74, developed by Celal Konor at CSU. This includes testing the code on NERSC supercomputers, providing 1-4 deep loop structures for testing on the processor core, and writing automated scripts to analyze looping and timing. Additionally, we are conducting an analysis of the full production implementation of the CSU icosahedral code on the NERSC Franklin system. Preliminary scalability analysis has already led to a number of changes to the icosahedral code to enable high-concurrency scaling.

\section{Hardware Design}

In early 2008 we finished a license agreement for a tool suite to synthesize custom processor cores. We then leveraged this toolset to created a baseline core design for the climate machine that includes a double-precision floating point unit with a predicted power consumption of just $\sim 0.09$ Watts and area of $\sim 1 \mathrm{~mm}^{2}$ for a $500 \mathrm{MHz}$ core in $65 \mathrm{~nm}$ chip lithography technology. Due to the complexity of the climate code a hardware emulation environment was created with the intention of eventually being able to benchmark the full climate code for the target ultra-efficient architecture using the UC Berkeley Research Accelerator for Multiprocessors (RAMP) system based on a BEE3 (Berkeley Emulation Engine) FPGA hardware emulation platform. Additionally, we have investigated a broad range of power-efficient memory technologies to maximize the power efficiency of the system design.

\section{Autotuning}

The research effort also examined the limited area icosahedral code and expanded the scope of our autotuning framework to automatically transform key loops in the search for the optimal performance of processor core. A key innovation of the prototype design is to enable tightly coupled hardware/ software co-design, which combines software autotuning together with hardware design space exploration to achieve a more robust and power-efficient design point than current design practices. Initial work shows a 20\%-200\% performance improvement over the baseline processor core performance and up to a factor of 10x improvement in power efficiency over conventional "power efficient" hardware designs such as BlueGene. A study of this approach has been submitted to HPCA 2009. This co-design approach will be a key design tool for automating the optimal design point search of power-efficient computing system for science. 


\section{Integrated Performance Monitoring: From Application to Workload Principal Investigator: David Skinner (Lawrence Berkeley National Laboratory)}

\section{Project Description:}

Monitoring the performance of scientific computation through passive profiling provides a reasonable middle ground to accommodate the needs of the science users and center managers in getting the most out of large scale scientific compute resources. This LDRD increases the coverage of Integrated Performance Monitoring (IPM) across HPC workloads via a two pronged approach. 1) Increasing the applicability of IPM to the segments of the HPC application space which are currently not covered. 2) Improving the backend infrastructure of IPM so that it can be used for quantitative analysis of the center-wide workload.

These two goals reinforce one another and have been pursued in parallel. This LDRD has performed somewhat risky software development and research to extend IPM to new areas of scientific applications. It has also enhanced the generality of IPM by encouraging funding and collaboration outside the NERSC center. IPM work with POSIX IO is now funded through both DOE and NSF.

Accomplishments:

Through this LDRD we added profiling for both parallel POSIX I/O at scale as well as data transfer profiling of WAN data movement through grid-ftp. Our research into WAN bandwidths had very few surprising results. The POSIX I/O work has opened a new area of research which is quite complex and currently being investigated. The end-to-end bandwidth visible to the application is a convolution of the underlying hardware, filesystem, I/O library, the workload running on the machine and the application algorithm. Understanding the application level profile is thus complex and actively being studied at NERSC. Similar studies are underway at LBL and SDSC.

The POSIX I/O profiling extensions to IPM have been implemented and are now in use by HPC researchers on NERSC Linux, Cray, and IBM parallel machines. It was found that the rate at which $\mathrm{I} / \mathrm{O}$ calls are made is significantly lower than the rate at which MPI function calls are made. As a result we have been able to collect both profiles as well as full traces of several DOE applications. Such profiling reveals the importance of I/O performance in addition to communication and computation. This software is now in use by HPC researchers to optimize large scale parallel filesystems and applications that run on them. 
Reference Benchmarks for the Dwarfs

Principal Investigator: Erich Strohmaier, HPCRD

\section{Project Description}

We propose developing a set of benchmarks representing important groups of algorithms known as 'dwarfs'. Each dwarf captures a pattern of computation and communication common to a class of important applications. Thus, each dwarf represents a class of algorithms with similar properties and behaviors. These benchmarks will include realistic data set definitions spanning from desktops to HPC systems. Such a set of benchmarks could serve as an important focal point of research for years to come strongly influencing the future direction of the hardware and software industries. This benchmark set will enable research in a variety of areas such as onchip and off-chip computer architectures, energy efficient computing, parallel programming paradigms, compilers, and system level software.

To avoid preordaining parallel codes, we plan to develop pencil and paper description for each algorithm, and create realistic, scalable problem descriptions and input datasets to enable experiments from single sockets up to full scale HPC systems. We will provide means to verify proper and accurate program execution and we will also develop reference implementations in high-level languages such as MATLAB to demonstrate the algorithms in a simple fashion but with no performance expectations.

To promote energy efficient computing, we are also researching new methodologies for ranking supercomputers to augment rankings such as the TOP500. This includes the use of alterative benchmarks as well as new metrics, which include effects of power and resource consumption of computer systems.

\section{Accomplishments}

We developed new methodologies to include power consumption in ranking supercomputers and to augment the current ranking of the TOP500 project, which is purely based on the Linpack benchmark performance. We collaborated with the TOP500 project to extend the type of system information collected, so that our new metrics could be used for alternate rankings. In addition to achieved performance, the new ranking system uses the available memory size and power consumption of a system for calculation of a generalized utility metric. We also formulated an extended version, which includes space consumption.

We started to assemble an in-depth overview of the scientific computing dwarfs. For this we describe for each dwarf the main algorithms currently used solving problems covered by it, and then analyze in detail the architectural requirement of these algorithms for computation and communication. We classified several set of widely used benchmark groups such as the HPC Challenge Benchmark and the NAS Parallel Benchmarks within the framework of the dwarfs to determine coverage of the dwarf space by existing benchmarks.

Optimization of benchmarks is an often necessary but potentially cumbersome step. Autotuning techniques on the sources code level can eliminate the burden associated with optimization and generate set of benchmark results suitable for fair comparison of architectures. We characterized previous efforts of kernel auto-tuning according to the dwarfs and found substantial but not complete coverage of this space by auto-tuning efforts. We therefore started an auto-tuning effort for kernels selected from some of the missing classes of dwarfs. 


\section{Extended First Order System Least Squares Finite Elements Principal Investigator(s): Jon Wilkening}

Project Description

The purpose of this project is to develop numerical methods in the least squares finite element setting for adjoining special basis functions to the finite element space in order to capture features of the solution that are not accurately represented by polynomial basis functions. The first step is to devise a method of constructing stable representations of singular basis functions describing the asymptotic behavior of the solution of an elliptic system near a corner or interface junction. The second is to develop an algorithm for assembling the stiffness matrix that computes inner products with the singular basis functions accurately and efficiently. Finally, we seek new problems that can be studied using this method directly, or using the tools we developed for this project in new ways.

\section{Accomplishments}

We have completed 8 papers this year related to this work. In the first paper listed in the publication list, we describe our method of adjoining singular basis functions in the FOSLS framework for isotropic linear elasticity and the Stokes equations. The key idea is that we can compute the stiffness matrix without employing special purpose quadrature rules for overlapping singular functions in the corners. This is possible because the singular functions satisfy the PDE; hence, they are invisible to the FOSLS functional except in the fringe region, where they transition to zero. But in this region, they are not singular and can be integrated using the same Gaussian quadrature rules used for the regular basis functions. Much of the paper deals with correctly applying boundary conditions at corners and triple junctions where some components of the stress tensor are singular.

In the second paper, we discuss the construction and stabilization of power solution bases by defining a Riemannian metric with respect to which we orthogonalize the basis functions before using them in our finite element calculations. Correct linear combinations of the pure power solutions are automatically chosen to recover the logarithm terms at certain critical angles. We show how to remove degeneracy from the construction of Costabel and Dauge in order to obtain a 1-1 correspondence between self-similar solutions of the elliptic PDE and Jordan chains of a certain analytic matrix function. We exploit this correspondence in our construction of power solutions before stabilization.

In the third paper, we develop a new algorithm for computing quadrature schemes for arbitrary families of functions that improves on the method of Ma, Rokhlin and Wandzura by introducing an objective function with a unique minimizer corresponding to the desired set of abscissas. Our trust region approach is much more robust than the previous approach and avoids the need for hand-tuned numerical continuation methods to get close enough to the solution for Newton's method to converge. This paper was motivated by the need to orthogonalize the power solution bases discussed above.

In the fourth, fifth and eigth paper, we study bifurcation of time-periodic solutions of the Benjamin-Ono equation. Our interest in bifurcation theory arose in the context of critical angles at which the asymptotic form of corner singularities of elliptic systems change type. In the sixth paper, we extend our algorithm for computing Jordan chains (published in 2007) to an algorithm for computing Smith normal forms of matrix polynomials. In the seventh paper, we develop a variational particle scheme for non-linear diffusion problems and gas dynamics; the minimization algorithm in this code is closely related to the trust region approach we developed for computing quadrature schemes. 


\title{
Earth Sciences Division
}

LB07008

\author{
Applications of Adjoint Field Methods and Time-Reversal Data Processing to \\ Inverse Problems in Electromagnetics, Seismics, and Ultrasonics \\ Principal Investigator: James G. Berryman
}

\begin{abstract}
Project Description
Research in complex geophysical imaging situations has shown that a robust method of attacking many imaging and target localization problems confronting geoscientists is the adjoint field method. This method had appeared early in reflection seismic imaging for oil industry applications, in part because the enormous size of the data sets had precluded the use of any other method. This approach and its generalizations have considerable value in many applications including nonlinear imaging problems in ultrasonics and electromagnetics, as well as seismics. In particular, the related method of time-reversal data processing has been developed for diagnosis and treatment in biomedical applications and underwater for secure submarine-to-submarine communications, as well as for more traditional ultrasonic imaging in NonDestructive Evaluation (NDE).
\end{abstract}

Time-reversal data processing is one example of the recent progress made in successful uses of this very general adjoint field method. Two areas of applications being pursued here are (1) electromagnetic target location and identification in the earth, and (2) methods of focusing sound and/or seismic waves in spatial regions where increased sound pressure can result in directed motion of fluids for either biomedical or oil industry applications.

Accomplishments

Studies are in progress for enhancing current LBNL methods for UXO (unexploded ordnance) detection and identification with the time-reversal processing ideas. This approach leads to a computationally useful data decomposition into symmetric $3 \times 3$ complex matrices that can result in increased data processing speeds for field applications, and may lead to advantages for hand-held (much smaller than the current version) UXO detection and identification devices.

A method for focusing either ultrasound or seismic waves that results in a tool for forcing fluids to move in a user-specified direction was been developed. A paper developing these ideas has been written, as well as a patent disclosure. The paper has not yet been submitted for publication while we are waiting to get the patent application to be finalized (expected to happen in late December, 2008) and then submitted. This idea is especially useful for biomedical applications where one needs (for example in the human brain) to move drugs around the body with as little physical invasion (by catheters, needles, etc.) of the targeted region needing the drug therapy as possible. Similarly, in earth sciences, the pertinent applications include dislodging of stuck oil, and/or of forcing underground contaminants towards a collection point. Current methods used in the earth sciences applications are necessarily very time and energy inefficient, as there has been no obvious way to focus the seismic waves produced by surface seismic vibrators towards the underground regions of most interest. 
Lattice-Boltzmann investigation of isotopic fractionation during mineral precipitation Principal Investigator: John N. Christensen

\section{Project Description}

Virtually every geochemical process in nature involves chemical exchange between solid minerals and fluids. In almost all cases this exchange takes place under non-equilibrium conditions due to transport limitations not only in the fluid phase, but also at the mineral interface, and within the solids. Because of the mass-dependence of transport properties, and different vibrational energies, isotopic species are fractionated during chemical processes. Similarly, trace elements can be preferentially incorporated into crystals or excluded. In the earth sciences observations of such trace element isotopic fractionation in minerals are used, for example, to infer conditions of rock crystallization, or to reconstruct past variations in Earth's climate. Until now it has been difficult to establish to what degree the elemental and isotopic fractionations observed in nature and in experiments represent equilibrium versus transport effects. For example, analytical models, using simple geometries and boundary conditions, have proven inadequate to explain recent observations of heavy-isotope (e.g. $\mathrm{Ca}, \mathrm{Mg}, \mathrm{Fe}, \mathrm{S}$ ) fractionation. The goal of this project is to develop methods for more realistic and accurate simulation of mineral-fluid exchange, in order to better understand isotopic effects and the solid surface physicochemical environment.

In this project we have taken two approaches: (1) numerical modeling of crystallization processes, including incorporation of isotopic fractionation, using lattice-Boltzmann (LB) methods, and (2) experimental precipitation of calcium carbonate (calcite) to investigate $\mathrm{Ca}$ isotopic fractionation under a range of crystal growth rates and fluid dynamic conditions. The results of these laboratory experiments are also used to test the LB methods. We use the latticeBoltzmann method to simulate mineral precipitation under conditions of transport-limited growth with arbitrary geometry, and including both diffusion and flow.

\section{Accomplishments}

We have developed a 2D lattice-Boltzmann model for a single-component solid crystallizing from a moving fluid phase with that component in solution. As an example with a fundamental importance to Earth Science and climate studies, we built a LB model that simulates the growth of ice crystals and snowflakes in clouds (Lu et al. accepted Dec., 2008). The results of this modeling replicates some key features of ice crystal growth, and suggests that the spacing of the dendritic arms of snowflakes represents the lengthscale at which diffusive transport in the vapor phase is outpaced by variations in deposition rate along the crystal surface. We have developed an approach to simulating precipitation from aqueous solution involving two components forming a stoichiometric solid with charge balance. We have also incorporated isotopic fractionation into our lattice-Boltzmann models for calcite precipitation (Lu et al., presented AGU fall 2008) and ice crystal growth. We have also made progress in our laboratory experiments to investigate isotopic fractionation of $\mathrm{Ca}$ during crystallization of calcite. We have set up and tested an experimental apparatus with controlled $\mathrm{pH}$ and alkalinity, and controlled fluid flow conditions with a variably geared stirring system to access a range of Reynolds numbers. We have demonstrated that $\mathrm{Ca}$ isotopic fractionation of several per mil can be produced in our apparatus during calcite crystallization. Our preliminary laboratory experiments and our advancements in LB modeling provide a firm basis for our future research into the phenomenology of solid precipitation and isotopic fractionation. 


\title{
Integrated Earth Systems Climate Modeling and Analysis
}

\author{
William D. Collins
}

\section{Project Description:}

The effects of climate-change mitigation strategies on the integrated Earth system are not well understood. The main goal of this pilot project is to integrate mitigation with modeling and observation of the climate. Studies of biofuels have usually focused on the direct climatic effects from reduced emissions of long-lived greenhouse gases (LLGHGs). However, large-scale adoption of biofuels could also significantly reduce the amount of anthropogenic aerosols. Anthropogenic sulfate and carbonaceous species are the most important types of aerosol for cooling the climate system. Because of the short residence time of aerosols in the atmosphere, the introduction of biofuels could reduce the radiative cooling by aerosols in countries adopting this technology. The resulting increase in sunlight could appreciably affect regional temperature, precipitation, and carbon cycle dynamics. This project will investigate the effects of introducing biofuels on the physics, chemistry, and biogeochemistry of the Earth system.

\section{Accomplishments:}

We have established a collaboration with PNNL to use their latest emissions scenarios developed for the U.S. Climate Change Technology Program (CCTP). These scenarios represent projections of future emissions of greenhouse gases and other climate-changing chemical species for a variety of possible carbon-reduction technologies. We will use the subset of the scenarios in which biofuels constitute the primary mitigation strategy and will compare that to the new reference (business as usual) scenario.

We have completed work to introduce a new and far more accurate and extensible treatment of atmospheric radiative processes in the target climate model for this project, the Community Climate System Model (CCSM) version 4 [Iacono et al, 2008a,b]. This new treatment of radiation is designed to calculate the radiative effects of the major greenhouse gases on both terrestrial radiation and solar radiation. CCSM4 will be the first climate model worldwide to include such a complete calculation of radiative forcing of the climate system. For the purposes of this LDRD project, we are exploiting the new treatment to quantify the effects of biofuels on aerosol direct and indirect forcing including a much more complete treatment of the chemical, microphysical, and optical properties of the aerosols than has been previously possible. 
The Global Methane Cycle and Climate Change

Principal Investigators: Inez Fung and William Collins

\section{Project Description}

Methane is second only to carbon dioxide in its radiative forcing of climate change since the pre-industrial era and its budget is not well quantified. About $2 / 3$ of its sources are believed to be anthropogenic and are associated with wastages or inadvertent losses from energy and agricultural systems. Hence they are amenable to purposeful reduction and mitigation of climate change. The long-term goal of the project is to improve the prediction of climate and assessment of carbon management strategies by including methane-climate feedbacks in the global carbonclimate model. The immediate goals are to quantify the sources and explain the slowing of the methane growth rate in the 1990s; to study the radiative properties of methane; and to incorporate a prognostic methane cycle in a global climate model.

This project plans to (1) compile new energy, agricultural and socio-economic data to improve characterization of all methane sources, especially those related to inadvertent losses from energy exploration and delivery systems and from agricultural systems; (2) develop a new model of wetland methane emissions that has sophisticated treatment of wetland hydrology and biogeochemistry; (3) explore the possibility of future hydrate destabilization using TOUGH+/Hydrate and climate scenarios; (4) carry out 3-D atmospheric transport-chemistry model experiments to test hypotheses about the changing methane budget; (5) incorporate an interactive methane cycle in DOE-NCAR global carbon-climate model; and (6) carry out detailed radiative transfer calculations to study the effects of increased methane on solar radiation and radiative forcing of climate change.

Accomplishments

We have initiated an analysis of the variability of methane, carbon monoxide and methyl chloroform for the past two decades in an attempt to understand the common variability of $\mathrm{OH}$ oxidation, and to quantify the residual methane trends to be explained by anthropogenic sources. Using published methyl chloroform production rates, we determined that the $\mathrm{OH}$ trend at $\mathrm{Pt}$. Barrow, AK is 2-10 times larger than previously estimated. This suggests that the production rate for methane is smaller than previously thought.

We have also compiled statistics of anthropogenic methane sources. Area under rice cultivation has leveled in the past decade despite the increase in human population. Together with transitions from organic to industrial fertilizers and from flooded fields to intermittent irrigation, the methane emissions from rice cultivation are expected to decrease. However, methane emissions associated with enteric fermentation are expected to increase, as Brazil's cattle population (the second largest in the world) has grown by $3.7 \%$ per year in the past decade, while India's cattle population has remained relatively constant.

Our project is greatly aided by the World Bank report that details the methods for separating gas flares from other sources of nighttime lights. Global flaring of methane has remained relatively constant, albeit with large interannual variability, in the past decade.

One of our key objectives is to improve calculations of $\mathrm{CH}_{4}$ radiative forcing in climate models. We have collaborated with DOE ARM to introduce a new radiation code in the DOENSF climate model CCSM. The new code is: (i) based on the latest spectroscopic data; (ii) tested against line-by-line radiation code; (iii) validated against field observations. The new code produces much better estimates of $\mathrm{CH}_{4}$ radiative forcing and will be part of CCSM4. 


\title{
Compositional and Functional Analysis of Cell-Wall during Metal-Bacterial Actions
}

\author{
Principal Investigators: Hoi-Ying N. Holman, Ping Hu, Sharon Borglin
}

\section{Project Description}

The purpose of this project is to initiate the development of a dual imaging technology at the Advanced Light Source. It builds upon the novel tunable vacuum ultraviolet (VUV) nanoimaging of biomolecules (developed by the Ahmed Group) at the Chemical Dynamics Beamline and our Fourier transform infrared spectromicroscopy of live cells at the Infrared Beamline. These results will enable us to further the development of existing molecular microprobe tools for characterizing bioremediation of heavy metals. Data generated also would allow us to help creating computational tools to infer both immediate and long-term stability of these biologically transformed metal ions in subsurface environments, which will enable a more realistic assessment of sustainable bioremediation technologies.

We will develop a cell-wall-like system in which its physical and chemical properties can be rigidly controlled for investigating biomolecule-metal interactions. After establishing these model systems, we will use them as test beds for the development of the VUV nano-imaging, and for the extension of the existing infrared micro-imaging technology. We will test the existing instrumentation components and operation parameters, and will modify them if necessary. These data will also be used to extend existing molecular dynamic algorithms, which will enable a more in-depth analysis of the dynamics of an interacting system.

\section{Accomplishments}

We have successfully established the procedure for developing model bacterial cell-wall-like systems in which physical and chemical properties can be rigidly controlled. Here we design and build micro-stamps by the UV photolithograpy which enables us to rapidly generate model cellwall/membrane systems with controlled size and properties. This procedure has been tested by an electro-formation cell feasibility study. Here, we tested our ability to control the size and growth process of the model cell wall/membrane systems, and to insert macromolecules like membrane proteins, DNA, and polysaccharides into the systems. Initial results have demonstrate that we have achieved the ability to design and develop cell-like neutral/anionic/cationic unilamellar liposomes (as an outer-membrane system) for the future investigations of lipid-metal interactions, to design and develop cell-like giant unilamellar liposomes (as an outer-membrane system) for entrapping macromolecules and study their interactions with metal ions, and to design and develop cell-like giant unilamellar liposomes (as an outer-membrane system) for entrapping macromolecule-metal complexes and studying the long term stability of the complexes.

We also have successfully used the cutting-edge synchrotron infrared spectromicroscopy technique at the infrared beamline and the novel technique of the tunable vacuum ultraviolet (VUV) nano-imaging of biomolecules at the Chemical Dynamics Beamline to characterize the chromium species in different model biomolecule/chromium, cell-envelop/ chromium, and bacteria/chromium systems. Manuscript is under preparation.

Finally, we have succeeded in obtaining funding support from DOE to transfer our LDRD experience to study plant cell wall materials. 


\title{
Regulation of Carbon Partitioning in Grasses
}

\author{
Principal Investigator: Christer Jansson
}

\section{Project description}

Grass cell walls are likely to become a significant source of renewable biofuel as feedstock engineering and cell wall deconstruction technologies are moving forward. Dedicated bioenergy grasses such as switchgrass and Miscanthus, and stover from established agricultural crops like corn and sorghum, are all potential important producers of polysaccharides for downstream processing to ethanol, biodiesel, hydrocarbons, or other fuels. Increasing our understanding of grass cell wall formation and unravelling the regulatory networks that govern carbon allocation to cell wall synthesis, or to other sinks such as roots, are critical elements of the research necessary to obtain and utilize future bioenergy crops.

The purpose of this project is to further our understanding of the molecular mechanisms that control key enzymes in carbon allocation and partitioning in plants. The overall aim of the research is to elucidate the network underpinning the regulation of carbon allocation between sinks, and the partitioning of carbon to cellulose, hemicellulose, and starch synthesis during grass development. We study cereals such as barley, sorghum and rice and intend to sert up Brachypodium as a model grass system. The project also aims to employ synchrotron radiation-based Fourier transform infrared (SR-FTIR) spectromicroscopy (SR-FTIR SM) as a high-resolution analysis of structural and nonstructural carbohydrates in single plant cells.

\section{Accomplishments}

We have isolated a family of transcription factors (the SUSIBAs) and shown that they participate in sugar-signaling regulation of carbohydrate synthesis in source (leaves) and sink (endosperm) organs during cereal development, and we are further investigating their functions and activities in other grasses.

We have demonstrated the presence of an oscillator that regulates gene expression during starch synthesis in sorghum endosperm and cassava storage roots.

We have established antisense oligodeoxynucleotide (ODN) inhibition as a powerful diagnostic tool for gene function analysis in plant biology, and we continue to improve the method and are exploring novel applications for studying carbon partitioning. 
Microbiomics of Complex Microbial Communities in Environmental Samples

Principal Investigator(s): Professor Janet Jansson (Senior Staff Scientist, ESD)

\section{Project Description}

The purpose of this project is to understand the composition and function of complex microbial communities in environmental samples using molecular approaches, i.e. microbiomics, with a focus on key microbial processes of relevance to climate change, bioremediation and human health. In most environments microorganisms exist as complex communities that consist of thousands of different species. It is the composition and activity of these communities that determines the impact microbes will have on ecosystems and man.

We will apply molecular methods to characterize entire microbial communities in soil, sediment and the human gut and describe their individual components as well as their roles in various biogeochemical processes and interactions with the environment and man.

\section{Accomplishments}

One of our most significant accomplishments was the correlation of microbial community composition (and metabolites) to individuals with the ileal Crohn's Disease phenotype. We made a series of deep "omics" investigations on a set of fecal and biopsy samples collected from monozygotic twins with Crohn's disease. We completed 16S rRNA gene screening of fecal samples and biopsy samples from the twin cohort by terminal-restriction fragment length polymorphism (T-RFLP) and 454 FLX sequencing. In addition, we recently obtained the metabolite profiles from fecal water samples collected from the same set of twins with Crohn's disease, in collaboration with investigators at the German Research Center for Environmental Health. The next step is to correlate metabolites to specific populations.

We also successfully obtained the first human gut metaproteome from a set of healthy twins in collaboration with ORNL. This was the first study to demonstrate that it is possible to obtain high quality, extensive protein identifications from the human gut. Thousands of proteins were identified and some were found to represent previously undescribed pathways. Proteome samples from the twins with Crohn's disease are currently being processed.

We developed a novel technique based on microfluidics (Lab on a Chip) to sort bacteria from red blood cells on the basis of cell size.

In addition to our clinical research we have completed many other environmentally relevant studies. For example, we characterized the active bacterial community structure in polluted sediments and along vertical redox gradients in the Baltic Sea.

We recently completed the genome sequence of the 4-chlorophenol degrading bacterium, Arthrobacter chlorophenolicus in collaboration with JGI. We used the draft genome as a scaffold for proteomic investigations of $A$. chlorophenolicus under different growth states. 


\section{A New Technology for Permeability Enhancement for Natural Gas Extraction in Tight Reservoirs}

\section{Principal Investigator(s): Seiji Nakagawa and Andrea Cortis}

\section{Project Description}

With the dwindling supply of foreign and domestic oil and the push to reduce $\mathrm{CO}_{2}$ emissions, natural gas is destined to be one of the premier energy sources by the next decade. A significant part of technically recoverable gas is believed to be trapped within very low-permeability, "tight" gas reservoirs. The primary objective of this project is to develop an entirely new approach for enhancing the production of natural gas in tight reservoirs. The approach employs a selfgrowing, high-permeability "slot" that is driven from a borehole. The slot is advanced through a coupled hydro-mechanical process in which large stress concentrations around the borehole (or the advanced slot) in concert with high velocity fluid circulation combine to advance a permeable plane through a repeated process of stress concentration $\rightarrow$ rock failure $\rightarrow$ hydraulic debris removal. This method is potentially superior to the conventional hydraulic fracturing method to enhance formation permeability, because the orientation of the slot growth is optimal to encounter preexisting fractures.

\section{Accomplishments}

Previously we successfully produced a slot-shaped breakout in a small, low-porosity rock core using a low-tomedium-speed water jet (flow velocity $\sim 100 \mathrm{~m} / \mathrm{s}$ ). To make this method a practical technology we must answer two questions: Can we produce this type of breakout within natural, low-porosity sandstone? Can the breakout propagate far away (compared to the borehole diameter) into the rock? Large-diameter (4 inches) cores of natural sandstones were used for the experiment, one with a porosity of $26 \%$ (high-porosity) and the other with $12 \%$

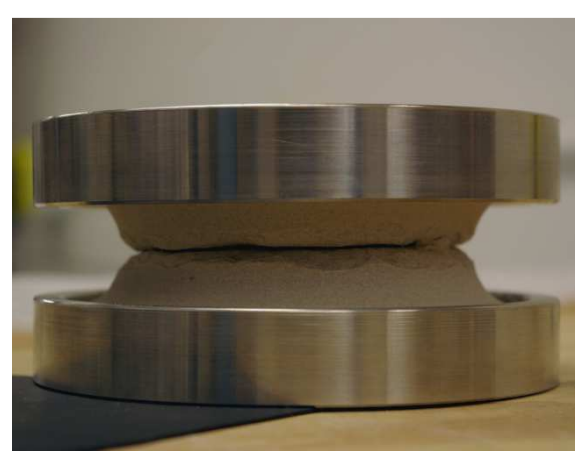

Fig.1. Slot-shaped borehole breakout produced in a low-porosity rock core (low-to-medium porosity). Using the same experimental setup as before, we applied a water jet to a borehole breakout produced in these cores. For the low-porosity rock, a narrow breakout was produced and propagated nearly halfway across the core (Figure 1), which strongly supports the possibility of producing a slot-shaped breakout for permeability enhancement in tight sandstone. In contrast, for the high-porosity sample, the growth of the breakout was not as prominent. This was against our prediction because a slot-shaped breakout should occur more easily for weakly cemented, high-porosity sandstones. The differences in the breakout propagation may be attributed to the mineral (sand) grain size and the size distribution. A microscope study on the grains indicated that the tighter sandstone core had relatively narrowly sorted (of similar grain size), fine sand grains while the higher-porosity sandstone showed a wide range of grain sizes.

As for the effort of numerical modeling of the slot-shaped breakout propagation, we encountered a numerous problems involving the difficulty of modeling high Reynolds number turbulent jet and the lack of computational power necessary for a large-scale granular medium model. An interaction of water jet and porous granular solid was examined for small models, using a non-linear (Navier-Stokes equation) finite element method via parametric variation of flow velocity. 
Coupled Modeling of Hydrology, Nutrient Cycling, and Vegetation: Applications to Water Quality and Water Balance

Principal Investigators: Curt Oldenburg, Bill Riley, Norm Miller (LBNL) Post-doctoral fellows: Federico Maggi, Chuanhui Gu (BWC)

Project Description

This joint Earth Sciences Division (ESD) and Berkeley Water Center (BWC) LDRD project is focused on understanding biogeochemical cycles of nitrogen species and production of $\mathrm{NO}, \mathrm{N}_{2} \mathrm{O}$, and $\mathrm{CO}_{2}$ gas and $\mathrm{NO}_{2}{ }^{-}$and $\mathrm{NO}_{3}{ }^{-}$solutes in natural and nitrogenrich agricultural fields, and the interaction between soil microbial biomass, the hydrological cycle, plant dynamics, and climate forcing. Motivations for this effort included improving our understanding of (1) $\mathrm{NO}, \mathrm{N}_{2} \mathrm{O}$, and $\mathrm{CO}_{2}$ emissions from the soil to the atmosphere as a key factor in climate change, regional air quality, and crop productivity, and (2) the effects of coupled water, carbon, and nitrogen dynamics on water quality and ecosystem function. These factors are assuming ever greater importance in light of expected increases in crop-based biofuels, food, and fiber production. To address these issues and goals, we developed a mechanistic model (TOUGHREACT-N) of $\mathrm{N}$ cycling in near-surface soils. We applied the model to investigate $\mathrm{C}$ and $\mathrm{N}$ dynamics in several managed and unmanaged ecosystems.

We developed the model TOUGHREACT-N (based on TOUGHREACT) for various nitrification and denitrification pathways, multiple microbial biomass dynamics, hydrological water flows, and various chemical reactions under local equilibrium and kinetic controls. The soil column is represented as a 1D vertical column with hydraulic properties described by a water tension/saturation model. Biotic and abiotic reactions are assumed to follow Michaelis-Menten kinetics, while biomass is assumed to follow multiple Monod growth kinetics accounting for electron donor, electron acceptor, and inhibitor concentrations. Water flow is modeled with the Darcy-Richards equation, while nutrient transport is modeled by Fickian advective and diffusive processes in both gaseous and liquid phases.

Accomplishments

We have tested TOUGHREACT-N against $\mathrm{pH}, \mathrm{NH}_{4}{ }^{+}, \mathrm{NO}_{2-}$, and $\mathrm{NO}_{3}{ }^{-}$ion concentrations, and $\mathrm{NO}, \mathrm{N}_{2} \mathrm{O}$, and $\mathrm{CO}_{2}$ gas emission data from sites in Sacramento, CA (Maggi et al., 2007), in Burgundy, France ( $\mathrm{Gu}$ et al., 2008), and Venezuela (ongoing). These tests resulted in improvements to the process model for $\mathrm{NH}_{4}{ }^{+} / \mathrm{NO}_{3}{ }^{-}$-forming fertilizers, urea hydrolysis, $\mathrm{pH}$ dynamics, $\mathrm{NH}_{3}$ volatilization, and $\mathrm{DOC}$ transport. We have also developed a preliminary mechanistic treatment of ${ }^{15} \mathrm{~N}$ exchanges in denitrification that can be integrated into TOUGHREACT-N as a process tracer (Maggi et al., submitted). Finally, we have coupled the model to the CropGro vegetation model, and to the land surface model CLM. The coupled CLM-TOUGHREACT-N model has been tested against the Hubbard Brook Experimental Forest and performed reasonably well on capturing stream $\mathrm{NO}_{3}{ }^{-}$dynamics (Gu et al., 2008). 


\title{
Design and Properties of Materials Involving Elements with High Neutron Cross- Sections That Are Useful in Neutron Detection and Related Neutron Technologies
}

\author{
Dale L. Perry
}

\section{Project Description:}

Materials containing elements such as $\mathrm{B}, \mathrm{Cd}, \mathrm{Gd}$, and $\mathrm{Li}$, which have isotopes with high neutron capture cross-sections, have extensive applications in neutron-related science and technology. This project involves theoretical and experimental investigations of the properties of materials containing such high neutron cross-sections and preliminary assessment of synthesis techniques. Particular emphasis is placed on materials with potential application to the detection of special nuclear materials (SNM's). The materials to be investigated are in the form of powders, crystals, composites, films, and adhesives. Techniques considered for synthesis include ambient temperature and pressure solution chemistry, ambient -temperature chemistry, and vapor deposition. Chemical forms to be investigated include mixed oxides, halides-oxides, and composites of both organic and inorganic components. Materials with applications for both current and projected programs in neutron science and technology have been considered.

\section{Accomplishments:}

During the course of this grant it was possible to (1) theoretically investigate composite semiconductor-organic nuclear radiation detection materials using different combinations of metal ion components; (2) evaluate the design of possible composite semiconductorsemiconductor nuclear radiation materials; using both exclusively metal as well as metalion combinations; (3) carry out spectroscopic studies of neutron-related nuclear radiation detection materials to show proof-of-concept for simultaneous determination of the concentrations and distributions of multiple elements of interest; (4) develop preliminary designs for gel materials containing elements that have high neutron cross sections; (5) carry out a feasibility study of neutron-sensitive metal ions for inclusion into nanoparticle photonic materials, and evaluated possibilities for the exchange of different metal ion hosts for neutron-sensitive materials.

The completed research resulted in contributions to five publications, and formed the basis for a proposal submitted to NA22. 


\section{Environmental Energy Technologies Division}

LB07014

Understanding the chemistry of innovative air cleaning technologies

Principal Investigator: Hugo Destaillats

\section{Project Description}

The goal of this project was to investigate the use of ozonation, photocatalytic oxidation, sorption and chemisorption treatments for the removal of volatile organic compounds (VOCs) from indoor air. Air-cleaning technologies employing these combined approaches can achieve important pollutant removal enhancements while extending the lifetime of catalysts and sorbents. Effective air cleaning devices may contribute significantly to improving the energy efficiency of buildings by reducing ventilation requirements. We intended to describe the underlying chemical basis by developing a technique for the characterization of gas-phase and sorbed reaction products and intermediate species generated in the reaction of model pollutants. This analysis would enable characterization the performance of innovative sorbents and catalysts designed using novel meso- and nanoscopically tailored materials.

\section{Accomplishments}

During the second year of this project, several research efforts have been completed. The Attenuated Total Reflectance (ATR) flow cell reactor was used for the irradiation of $\mathrm{a} \mathrm{TiO}_{2}$ thin film with UV light in the presence and absence of ozone, and to determine removal rates of parent compounds. The studied compounds included indoor air pollutants such as aromatic hydrocarbons, terpenes and cyclic siloxanes, as well as their oxidation products (e.g., hydroxylated aromatic compounds and carboxylic acids). In combination with XPS characterization, we identified surface species involved in inactivation mechanisms that affect the long-term performance of $\mathrm{TiO}_{2}$-based photocatalytic oxidation (UVPCO) air cleaners. We evaluated commercial catalysts (P25) and novel $\mathrm{TiO}_{2}$-clay nanocomposites synthesized and characterized specifically for this project. These materials showed a photocatalytic activity that was comparable with that of P25, and in one case (hectorite composites) a significantly larger active surface area for VOC adsorption.

Complementary insight into the photocatalytic process was gained from experiments performed on a second flow reactor built in our laboratory. In this reactor various catalyst materials were deposited on the surface of Raschig rings and irradiated with a UVC lamp. Toluene was used as a model VOC to characterize the performances of the reactor and of the photocatalysts under various experimental conditions, including UV frequency of irradiation, relative humidity and catalyst film thickness. This experimental system provided a framework for comparing novel photocatalysts with commercial products under realistically low concentrations, short residence times and other reactor parameters that are typical of air cleaner devices. Despite the numerous publications on this field, we found that the fundamental information generated in our studies was lacking, and will contribute to develop more efficient air cleaners.

An analytical method was also developed for the evaluation of the rate of consumption of a $\mathrm{NaMnO}_{4}$-based chemisorbent. This method allowed us to evaluate the performance of this reactive media under realistic operation conditions in buildings in concert with an UVPCO air cleaner. That information is critical to estimate the operational cost of this integrated method. 


\author{
Development of a Building Operating Platform \\ Philip Haves (PI), Alessandro Pinto, Alberto Sangiovanni Vincentelli, Michael Wetter, Yang \\ Yang
}

\title{
Project Description
}

The goal of this project is to test and demonstrate the applicability of platform-based methods for the design of control systems in buildings. These methods involve the use of systematic procedures to generate optimum designs for control systems based on formal specifications of the plant to be controlled, the performance requirements and the functional performance of the available components for constructing the control system. The potential benefits of successful implementation of the approach include (i) cost-optimal, 'correct by design' control systems for buildings, particularly energy-efficient buildings with innovative integrated systems, and (ii) replicability and traceability of building control systems, providing a mechanism for accountability for performance between designer, installer and operator. The project is linked to the Building Informatics Environment LDRD project, which will provide the simulation capability required for design and testing of building control systems.

\section{Accomplishments}

Several services of the Building Operating Platform (BOP) have been integrated and tested on manually generated case studies, including a case study of a temperature control system for the $5^{\text {th }}$ floor of Cory Hall. A model of the mechanical equipment was developed using the Modelica system simulation language. UC Berkeley’s Metropolis II (MII) software framework was used to integrate the simulation of the controllers, modeled as Finite State Machines, with the simulation the mechanical equipment.

In order to synthesize the network, the communication requirements are passed to COSI (Communication Synthesis Infrastructure), the network panning and analysis tool of the BOP. The controllers were manually mapped onto a model of the processor platform. When completed, the software synthesis service of the BOP will include a set of tools for doing this step automatically. For this task we have accomplished the following milestones:

- $\quad$ Literature review. The most relevant techniques for software synthesis that start from a formal description of the application to synthesize were reviewed, including methods for both centralized systems and distributed systems.

- $\quad$ Scope of the Control System Description Language (CSDL). A number of languages for implementing building control systems were reviewed in order to determine the scope required for the CSDL. It appears that all control programs of interested can be represented using a combination of the finite state machine and data flow models of computation

- Definition of inputs and outputs of the software synthesis process. Two abstraction levels were defined to support the transformation of a description of the control algorithm in CSDL to any of the existing control languages, taking into account the distributed nature of the implementation platform and performance and cost metrics.

- Hardware platform abstraction and formulation of the optimization problem. The optimization problem in software synthesis for distributed systems is to distribute the system such that the total cost is minimized while satisfying the end-to-end latency constraints from sensors to actuators. The distribution procedure includes task allocation, scheduling and communication. A pseudo satisfiability (SAT) solver was developed to solve the task allocation problem. 
Soot in Ice: Does Soot Enhance the Melting of Ice?

Principal Investigator: Thomas Kirchstetter

\section{Project Description}

The purpose of this project is to increase our understanding of how air pollution contributes to climate change. In particular, the focus of this project is the effect of black carbonaceous soot contamination on the optical properties and the melting of snow. General circulation and radiation models used in the prediction of climate indicate that the perturbation by soot in snow, which reduces the surface albedo (the fraction of incident sunlight that is scattered back to space), may be responsible for a significant portion of the global warming of this past century, including thinning of Arctic sea ice and melting land ice. A number of studies provide estimates of the amount of soot in surface snow, but there is little experimental or empirical data that support theoretical estimates of the radiative effects of soot in snow. This project will develop methods of making snow and soot-contaminated snow in the laboratory, wherein the amount of soot in the snow is controllable. In addition, this project seeks to measure changes in surface albedo and in the rates of heating and melting of snow exposed to light.

This project complements field measurements by other scientists and will allow for a "closure" study to be conducted between modeled and observed radiative fluxes of a fullyconstrained snowpack, either lending credence to predictions made with current climate models, or helping to improve future generations of climate models.

\section{Accomplishments}

Our most significant accomplishment has been the development of methods of making and measuring soot-contaminated snow in the laboratory. We generated soot with an inverted, co-flow diffusion flame of methane and air. The soot particles, which are almost entirely elemental carbon in composition, are initially hydrophobic. Through oxidation with ozone, the soot surfaces are transformed from a hydrophobic to a hydrophilic state, at which point they readily form a homogeneous suspension when mixed in water. We found ozonation to be highly effective in yielding the desired (electrostatic) stabilization, which prevents the soot particles from coagulating and settling. The soot suspensions are turned to mist using high-frequency vibration and the mist is cooled in a dry-ice chamber to make soot-contaminated snow. The amount of soot in the water suspension, and thus in the snow, can be varied by orders of magnitude.

We used a highly sensitive optical spectrometer equipped with a liquid waveguide capillary cell to quantify the mass of soot in water suspensions and melted snow at the low levels found in environmental samples - as low as 1 nanogram of soot per gram of water. The successful use optical spectroscopy to quantify soot in melted samples of laboratory snow suggests a viable alternative to other more cumbersome and error-prone techniques that are currently applied to samples of snow collected in the field.

Temperature-controlled chambers have been used to make and observe pristine and sootcontaminated ice samples. Our observations demonstrated the redistribution of soot when a homogeneous suspension of soot in water freezes: the soot becomes concentrated in the water that freezes last. This redistribution may have a significant impact on the evolution of the radiative forcing of soot in snow during periods of melting and refreezing.

We are in the process of developing a method using an integrated sphere to quantify changes to the snow albedo due to the contamination with soot. 


\section{China's Energy Future: Changes in Energy Intensity \\ Principal Investigator: Mark D. Levine}

Project Description

The topic of China's energy system is receiving increasing attention around the world. In many quarters, this attention has turned to alarm. Coal consumption is growing at an enormous rate (75\% growth in 5 years), power plants are being constructed at the fastest pace in history of any nation, and greenhouse gas emissions are growing apace. One-third to one-half of the world's incremental demand for oil has come from China in the past five years.

After a long and successful experience in implementing energy efficiency (1980-2002), China has seemingly lost its ability to carry out energy efficiency to scale. There is a clear need for a broad assessment of factors that affect the Chinese energy system as a whole and that have caused the disturbing change in energy intensity.

\section{Project Accomplishments}

The project completed the assessment of intensity changes in China's energy system. It produced the first and to date most authoritative analysis of the factors that have been of greatest importance in modifying the long-term trends of declining energy intensity observed from 19802002. Surprisingly, the trends of improved energy efficiency in industry continued. There was no discernible difference between the efficiency gains between 2002 and 2005 and those of earlier years.

The major change was the enormous increase in the output of heavy industry. Production of cement grew from 600 million tonnes in 2000 to 1.1 billion tones in 2005 . It has since risen to 1.4 billion tonnes in 2007 . This amounts to half of the world's production of cement. Steel production increased at an even faster rate and now amounts to more than $40 \%$ of world production. This astonishing increase in the output of heavy industry - for example, one year's increase in cement output in China from 2000 to 2007 is greater than the entire output of the U.S. cement industry - is a consequence of the rapid expansion of building and infrastructure. Such rapid expansion is expected to continue for years.

A second important finding is that China can achieve most, but not all, of its goal of a $20 \%$ reduction in energy intensity through efficiency. There is a need for a change in the structure of industry - a reduction of the growth in heavy industry, a goal that will be difficult to attain, and a speeding up in shuttering old, energy inefficient facilities - to achieve the policy goal.

The project also supported to study of energy demand forecasts for China, learning that all major forecasts of energy demand after 2002 for China were very low. Interestingly, both the international and the Chinese forecasts were equally in error. They all based their analysis on the continuation of historical trends, thus missing the enormous growth of heavy industry.

The two different analyses have the combined effect of pointing the way to improved methodologies for forecasting energy demand in China. It is this accomplishment that points the way to likely new avenues of research. 


\title{
Novel techniques to characterize secondary organic aerosols formed from gas-phase volatile organic compounds emitted from biogenic sources.
}

\author{
Melissa Lunden
}

Project Description:

The overall goal of this research is to advance current understanding of the processes that govern secondary organic aerosol (SOA) formation in the atmosphere. SOA is formed by the condensation of low volatility oxidation products of gas phase volatile organic compounds (VOC). SOA constitutes a significant fraction of aerosol mass in the atmosphere, and thus has important impacts on climate and visibility. Biogenic SOA is thought to be a dominant fraction of total SOA, yet emission inventories of biogenic SOA for climate models are the most simplistic and uncertain of the current aerosol inventories. The specific goals of this project are: (1) To develop novel techniques to analyze the chemical composition of SOA, (2) To investigate the composition and yield of SOA formed by oxidation of important biogenic species, (3) To develop techniques to make quantification of SOA in the field simpler and more economical, and (4) To facilitate future improvements to process models of biogenic SOA formation in climate models. These models will prove crucial as the world moves towards a future that replaces fossil fuels with those derived from biogenic sources.

The approach of the project is to characterize biogenic SOA formed in chamber experiments utilizing living samples of important biogenic species. The VOC emissions from the plants will be oxidized at a variety of atmospherically relevant conditions. The condensed aerosol oxidation products will be analyzed utilizing Fourier Transform Infrared (FTIR) spectroscopy and tunable VUV $(8-20 \mathrm{eV})$ photoionization techniques available at the Chemical Dynamics Beamline (9.0.2) of the Advanced Light Source (ALS). FTIR techniques allow for quantifiable measurement of all condensed organic compounds by chemical functionality. The aerosol endstation of the Chemical Dynamics Beamline utilizes a "soft" ionization technique to yield near fragment free mass spectra of large organic molecules allowing the chemical composition of SOA to be determined as never before. When combined, the FTIR and VUV techniques allow for SOA mass from biogenic sources to be characterized with unprecedented detail.

Accomplishments:

The primary accomplishments in the first year include the construction of a flow through aerosol reaction chamber and the use of that chamber to characterize aerosols formed from biogenic precursors. Laboratory generated aerosol were generated by reacting simple biogenic volatile organic carbon precursors such as d-limonene and alpha-pinene with ozone. The resulting particles were characterized using on-line aerosol instrumentation as well as collected for off-line analysis. The analysis was conducted using an FTIR acquired specifically for this project, and configured to characterize organic aerosol collected on filters. Analyses performed on the filter samples showed functional group data that would be expected from biogenic secondary organic aerosol. The spectrum did look significantly different from aged organic aerosol collected in a number of urban and rural areas around the world, which show significant anthropogenic influence. Work continues to use this successfully characterized laboratory generated aerosol to test automated sampling schemes for the FTIR that are in development. In addition, an automated collection device is being built to facilitate sample analysis at the ALS. 


\author{
Integrated Decision Support Tool for Joint Optimal Control of \\ Energy and Water Systems under Uncertainty \\ J.E. McMahon ${ }^{1}$, L.L Dale ${ }^{1}$, J.C. Meza ${ }^{2}$, P.O. Schwartz ${ }^{2}$, \\ B.A. Faybishenko ${ }^{3}$, N.L. Miller ${ }^{3}$, S.A. Finsterle ${ }^{3}$, D. Sunding ${ }^{4}$ \\ ${ }^{1}$ EETD ${ }^{2} \mathrm{CRD}^{3} \mathrm{ESD}^{4} \mathrm{UCB}$
}

\title{
Project Description
}

In this project we investigated water-energy trade-offs and the impact of changes in climate, electricity prices and water supply on resource management. We focused on the water supply process from reservoir storage to conjunctive groundwater storage, irrigation, and delivery, and provide a systematic estimate of the value of water and energy conservation. We also evaluated net energy requirements with water use options, including water for ethanol production. We developed decision support tools using dynamic programming for optimal control of energy and water systems. We are developing an integrated decision support tool from existing energy and water models, used explicit characterizations of sources of uncertainties, and developed a ranking of key uncertainties as targets for further research. Finally, we are presenting our results in a series of papers and conference presentations as a way to transfer this information to researchers, California agencies, U.S. agencies, and international programs. The results of our work demonstrate the improvement in energy and water use that is possible through optimal control of integrated water systems.

\section{Accomplishments}

The LDRD includes three major areas of focus, including theoretical/ mathematical accomplishments, modeling applications and policy studies. Theoretical accomplishments of the LDRD include idealized constructs of optimal conjunctive use of surface and ground water and optimal "anticipated learning" and option value given climate-change. New computing algorithms for building surface water storage, given anticipated changes in hydrology, were also developed.

Modeling applications of this LDRD include models of the Merced River Basin and of the San Joaquin Valley. The Merced River model estimates optimal reservoir and aquifer management as a function of water and electricity prices. The San Joaquin Valley model simulates the impact of changes in hydrology and cropping patterns on groundwater storage and long-run pump electricity demands.

Policy applications of this work include estimates of the impact of changes in water and energy prices on Merced basin water storage, San Joaquin Valley water supply and Central Valley crop patterns. 


\section{LB08038}

Using New Microbial Assays to Characterize Dampness-Related Exposures:

Combining Molecular Biology with Building, Aerosol, and Health Science Principal Investigator(s): Mark J. Mendell

\section{Project Description}

The overall goal of this project is to test a hypothesis that wet surfaces in air-conditioning (AC) systems emit small microbial particles, not previously measured, that are associated with potential health effects. The specific goals are: 1) To identify microorganisms on wet AC surfaces (cooling coils and drip pans), using DNA-based assays, under normal conditions vs. with ultraviolet germicidal irradiation (UVGI), in order to define sources for emitted microbial particles; 2) To identify any microorganisms on $\mathrm{AC}$ surfaces with prior known immunologic or allergic effects; 3) To define concentrations of airborne particles emitted from wet AC surfaces, with and without UVGI; 4) To assess inflammatory and antigenic properties of microbial particles emitted from AC surfaces, and identify particles by species as feasible; and 5) To facilitate future epidemiologic studies on health effects from damp AC surfaces and other damp indoor surfaces.

In the first year, this project planned to 1) use new DNA-based assays to identify bacteria and fungi on wet $\mathrm{AC}$ coils in two buildings - a hot-summer east coast building with prior demonstrated health effects from AC-surface microbes, and a hot-summer California building; and 2) in the California building, study particles emitted into the AC air stream - identify species and assess inflammatory and toxic potential. In the second year, the project plans to 1) count, sample, and analyze size-specific fractions of the airborne microbial particulate emissions, including respirable and ultra-fine fractions; 2) determine, for size-specific fractions, number concentrations and inflammatory and cytotoxic effects, and, to the extent feasible with DNAbased methods, the identities of fungal and bacterial particles emitted from wet AC surfaces; investigate the effects of ultraviolet irradiation in reducing microbial emissions from $\mathrm{AC}$ surfaces, in either an occupied building or a controlled AC system at LBNL.

\section{Accomplishments}

We developed a successful sampling method for AC cooling coil fins in a hot-climate building. From among metal plates and foil of many thicknesses, cytobrushes, and swabs of many materials and shapes, the only suitable methods were flocked nylon urethral swabs for the fins, and either those or polyurethane foam swabs for pans.

We retrieved material from three locations in the AC system: fins in the front and back of coils, and the drip pan, when both coils and pans were wet. We successfully extracted DNA from three samples - from the front and back of cooling coils, and the drip pan - using solvents and bead-beating; amplified the DNA with multiple PCR cycles; and identified over 300 bacterial "observational taxonomic units" (OTUs) with the Phylochip DNA microarray. We are currently exploring specific OTUs found, and differences between the sampling locations.

We have prepared a system using the human lymphoma-derived cell line U937, converted to macrophage-like cells by treatment with phorbol-12-myristate-13-acetate (PMA), as a model for human cellular response to inflammatory stimuli. We have successfully tested the system with lipopolysaccharides (LPS) and assayed release of IL-8 over time. We also will use IL-6 assays.

We are now planning air sampling in $\mathrm{AC}$ systems, and our next analyses of both $\mathrm{AC}$ surfaces and air samples, that will include the DNA-based bacterial identification assays, DNA-based fungal identification assays, traditional cultural identification assays for both bacteria and fungi, and the cellular assays of inflammatory potential. 
Using IP Telephony and Wireless Technologies to Extend the Reach of Conventional Building Automation Systems

Principal Investigator(s): Francis Rubinstein

\section{Project Description}

The project objective is to demonstrate the energy efficiency, demand response and comfort consequences of applying wireless communications and IP telephony to advanced building automation systems (BAS). We will test different emerging open platforms for developing applications that can the integrated lighting and shading "proofof-concept" system developed in the FY08 research.

We will evaluate middleware platforms for cell phones and PDAs, to evaluate the reliability of mobile devices for: 1) communicating with BAS, 2) informing building facilities managers as to grid emergency conditions and real time energy prices, 3) and for commissioning and maintaining automation systems. IP phones and wireless controls represent a plethora of additional pathways for occupants to personalize their environment and building managers to override facility-wide control settings, especially in existing buildings where re-wiring is prohibitively expensive. We will investigate whether miniature wireless controllers can serve as practical intelligent nodes for deploying BAS services in existing buildings. Finally, we will demonstrate the integration of the matchbox controller with the Building Operating Platform (BOP) under development in the HiPerBRIC Project.

Accomplishments

Our most significant accomplishment was the testing of a "proof-of-concept" integrated lighting and shading control system capable of implementing a detailed building control use case that increased energy efficiency, improved demand response, and improved user access to lighting and shading services. We installed the proof-of-concept system, which consisted of a LabView-based data acquisition and control system with user overrides operating dimmable lights and motorized venetian blinds, in a test room at B46-232. A control algorithm was developed to execute the integrated building control use case and was encoded into LabView. The amount of daylight entering the space was modulated using a control algorithm that operated the motorized Venetian blind system. We inputted the signal from a masked photometer with an exterior field of view into the daylighting control algorithm to control the blade angle and retraction distance. The daylighting algorithm attempts to keep glare below the discomfort level while maximizing openness and view. We tested the proof-of-concept over several nonconsecutive days under clear sky, partly cloudy and overcast conditions.

We verified appropriate operation of the shading system. With one exception, the blinds changed height twice a day or less, meeting the goal of avoiding unnecessary fluctuations in position that might be bothersome to the user. In every instance that the blinds did change height or tilt, the system was performing according to a discomfort glare threshold. Appropriate response of the dimmable lighting control system in response to light levels measured by interior photocells was also verified.

The control algorithms and processes developed in this research have positioned us to extend the work to explore phone-based overrides for advanced BAS and for porting over to miniature wireless controllers that we believe can serve as practical intelligent nodes for deploying area-based building automation services in existing buildings. 
Software for Integrated Analysis of Sensor Data for Advanced Energy Controls Principal Investigators: Michael D. Sohn and Ashok J. Gadgil

\section{Project Description}

The purpose of this project is to develop algorithms for analyzing the data that stream in from diverse sensors, for real-time assessment of building energy performance, occupant comfort, and fault detection. The research joins recent advances in Bayesian statistics, decision analysis, and physics-based energy modeling. The research will result in software that may be applied to assist in designing sensor networks (e.g., identifying what information is needed, and from where). The software also may be used to interpret data from an existing sensor network, in order to automatically tune the energy management and control system (EMCS), flag hardware that might have become defective, and maintain optimal comfort while minimizing energy consumption.

The proposed software will be, therefore, a statistical analysis tool that can take inputs from a data-collection network and a physics-based simulation tool, and provide as output various analyses, decisions, and action items for overall building assessment and control. It will allow a user to compare predictions of energy use against real measurements, and will improve on current EMCS systems by merging/integrating data from diverse sensors and multiple subsystems, with physics-based simulations. The real-time feedback will also help confirm successful installation of new equipment or sensors, detect unusual operating conditions (e.g., a stuck damper or a jammed fire door), and suggest contingency responses during emergencies (e.g., to avoid unsafe areas while evacuating during a fire).

Accomplishments

We have made significant progress in: (1) developing the conceptual and statistical algorithm for "fusing" data from multiple sensors simultaneously, in real time; and (2) identifying/securing a real building for testing and development.

We examined a number of methods for analyzing sensor data, including Kalman filtering, linear and non-linear regression, and stochastic modeling, to determine which are suitable for analyzing data containing errors (e.g., poor calibration or communication drop-outs) and uncertainties typically found from sensors in large commercial buildings. Through testing, we decided that the best operational capability will be an amalgam of these techniques, and that a particular statistical approach, called Bayesian Melding, is the likely best approach. Bayesian approaches manage errors in data and models well, and have methods for processing uncertainties, variability, and model-misspecification problems in physics-based energy models during an analysis.

We also located a building on the UC Merced campus where we can gather suitable monitoring data to develop and test our algorithms, and assembled a multi-organization research team to study it. The team comprises Professor Alberto Cerpa (UC Merced), Dr. Andrezej Banaszuk (United Technologies Corp.), John Elliott (UC Merced), and Professor David Auslander (UC Berkeley). Finally, the LDRD has helped us with a key new hire at LBNL.

For the second year of this project, we plan to further develop and test the algorithms using data from the UC Merced building. 
Building Informatics Environment Enabling Rapid Prototyping and Model Extraction for Building Automation Systems

Principal Investigator: Michael Wetter

\section{Project Description}

The purpose of this project is to prototype a Building Informatics Environment (BIE), a software environment for modeling and simulation of building energy and control systems. The BIE will enable rapid virtual prototyping to support the invention of new building energy and control systems. It will also allow embedding physics-based models into the Building Operating Platform (BOP), which is a software environment for the design, deployment and operation of building automation systems.

Using simulation-based virtual prototyping, the BIE will enable designers to explore a larger set of design options than in a hardware test-bed. It will also allow analyzing the dynamics of integrated systems during all seasons and with different boundary conditions, and hence, designing a robust system that can subsequently be tested in a hardware test-bed to include effects that are not explicitly modeled. The BIE will enable extracting models used during design and embedding them within the BOP in order to couple them with sensor networks for commissioning, model-based controls, fault detection and diagnostics.

\section{Accomplishments}

We developed a modular library with component models for building energy and control systems using the acausal object-oriented language Modelica. The component models are encapsulated using the same interface as their corresponding actual physical objects, and they can be assembled to form a system model in the same way as an experimenter would assemble real components in a laboratory. This assembly may be done manually by a modeler or automated by the BOP. It is enabled by acausal, standardized model interfaces and a model networking capability that imposes interface conservation equations and equates states and signals as needed. This allows model reuse in different applications. It also allows rapid modification of existing models and addition of new models without requiring changes to the system model topology, as would be needed, for example, in a block-diagram editor.

Proof-of-concept studies have been conducted for controls design and for pre-commissioning of a control system. In the first study, we designed a controller of a nonlinear plant using model linearization, reduced order model extraction, and frequency domain analysis. In the second example, a non-convex optimization problem with state constraints has been solved to find optimal control parameters. Both applications are outside the capabilities of traditional building simulation programs. 


\section{Genomics Division}

LB06033

Computational and Experimental Testing of Methods for Binning Sequences from

Metagenomic Studies

Principle Investigator: Jonathan Eisen

\section{Project Description}

Metagenomics is the study of the genomes of many microbes in an environment simultaneously, and has the potential to revolutionize our understanding of the hidden yet incredibly important world of microorganisms. The great potential of metagenomics comes with enormous challenges in the analysis of the data. One of the major difficulties lies in the fact that current methods produce data that represents only tiny fragments of the genomes of organisms and which genome each fragment comes from is not known. This is akin to one trying to reconstruct a library if someone took all the books, shredded them, and then gave back mixed up fragments of the shreds. Assigning the small pieces of genomes to organisms is something known as "binning," analogous to shreds to specific books, and is critical to making the best use of metagenomic data. Though there are several available methods of binning, there has been very little systematic work on determining how well these methods work.

The purpose of this project is to test known as well as new binning methods. This testing will be done using metagenomic data sets where the origin of sequences is known. By knowing the true origin of DNA sequences, we can then test various metrics for assigning sequences to bins. A key part of this work was to collect and generate three types of model data sets: computer simulations, metagenomic projects from low diversity systems, and in vivo simulations in which we mixed cells from organisms with completed genomes.

\section{Accomplishments}

We have three main accomplishments as a result of this project.

We assessed various computational methods for simulating metagenomic sequencing as part of a project designing new binning methods (Chatterji et al. 2008). Such simulations are critical because they provide one with data for which the answer is known and which then allows one to assess the quality of binning methods.

We have created the first in vitro metagenomic simulation whereby we mixed together cells from 10 different species and then took these mixtures through various metagenomic sequencing protocols. Analysis of the data is under way (Morgan et al, in preparation) and preliminary results indicate that these simulations help better understand real metagenomic studies because they produce significantly different patterns than seen with computational simulations.

We designed computer software for carrying out a critical step in analysis of microbial community data by the grouping of sequences into clusters of a set level of relatedness. Such clusters, known as Operational Taxonomic Units, or OTUs are critical for studies using metagenomic data. A paper describing this software, "OTUHunter" is in preparation (Huntemann et al. in preparation). 


\section{Life Sciences Division}

Functional Interactomics: Integrating Physical and Functional Interaction Networks Principal Investigator: Gareth Butland

\section{Project Description}

Interactions, both physical and genetic define the organization of the cell. Physical interactions, such as protein-protein interactions, help us understand how individual polypeptide chains come together to form protein complexes which perform many of the biochemical reactions in a cell. Other types of interactions, such as functional interactions, propose organizational relationships between gene products and protein complexes within the cell. Epistatic (genetic) interactions, one of the most commonly encountered forms of functional interaction, exist between genes which encode proteins involved in parallel pathways or processes. These interactions, whilst not physical in nature, provide information regarding functional redundancy within the cell and help understand the basis of suppressor mutations and synthetic lethality. This LDRD proposal is designed to develop and implement a methodology capable of screening for functional interactions in E. coli on a genome wide scale.

Classical genetic approaches have often combined null alleles in the same strain to assess if a functional relationship exists between two or more genes of interest. These experiments have however usually been restricted to specific biological niches in order to limit the number of null mutations that have to be made sequentially by laborious methods. This proposal will utilize the recent availability of a complete set of $\sim 4000$ single gene knock-out (KO) strains of $E$. coli. A null mutation in a gene whose genetic interactions are to be screened will then be combined in a pair wise manner with all 4000 mutant strains present in the KO collection and the viability of the strain bearing both mutations assayed. The key step of quickly and easily combining null mutations between strains of E. coli in a manner amenable to high-throughput analysis will be developed based on the natural genetic system of conjugation. This will facilitate the rapid screening of thousands of potential functional interactions in a rapid and efficient manner.

\section{Accomplishments}

Over the last year, we have completed our proof-of-principle work establishing the technology with which to screen for genetic interactions in E. coli, by systematically combining deletion mutations. In collaboration with colleagues at the University of Toronto, we have demonstrated the ability of this technology to uncover novel genetic interactions in the area of iron sulfur cluster biosynthesis, a topic of intensive research in recent years.

Mutations for all genes in both major E. coli pathways for FeS cluster biosynthesis, the Isc and Suf pathways have been combined against deletion mutations present in the E. coli single gene knockout collection and viability of double mutants assayed. Novel genetic interactions, such as the synthetic lethality of Isc pathway mutants in combination with the monothiol glutaredoxin GrxD, have implicated it for the first time as having a key role in bacterial FeS cluster biosynthesis. This highlights the power of this genetic interaction screening approach for uncovering novel functional relationships. This proof-of-principle work was published this year in Nature Methods. We are currently extending this analysis to complete a genetic interaction profile of all $E$. coli FeS cluster biosynthesis components. 
Heavy-Atom, Ultra-fast-Scintillator for Time-of-Flight Positron Emission Tomography Principal Investigator: Stephen Derenzo

\section{Project Description}

The goal of this project is to develop a revolutionary class of scintillator materials that will dramatically improve the capabilities of positron emission tomography (PET). It exploits an ultra-fast scintillation mechanism discovered by this research group at LBNL. In this mechanism holes produced by ionizing radiation in a direct-gap semiconductor are trapped on one impurity atom and then rapidly recombine with electrons from a second impurity atom to produce a flash of light that can be detected with extremely good timing accuracy. This accuracy will allow the difference in the time-of-flight of the two $511 \mathrm{keV}$ annihilation photons to localize the point of annihilation and greatly reduce the statistical noise in the reconstructed images. This also maximizes achievable data rates and the rejection of random coincident backgrounds. Moreover the photon yield of a semiconductor scintillator can be much larger than conventional scintillators, and this will result in improved energy resolution and an improved ability to reject tissue-scattered annihilation photons.

These improvements will allow diagnostic imaging studies that are impossible with conventional PET, such as developmental abnormalities in children, metabolic abnormalities in obese patients, very small lesions in metastatic cancer, and abnormalities in low-abundance neurotransmitter binding sites in the brain.

\section{Accomplishments}

During the one year of LDRD funding 71 crystalline samples of $\mathrm{PbI}_{2}$ crystals were prepared by the vertical Bridgman technique. All crystals were characterized for their scintillation properties at cryogenic temperatures and at room temperature. The wavelengths of the luminescence emissions were measured by photoluminescence and the decay times of the luminescence were measured by pulsed x-ray excited luminescence. Most crystals were doped or co-doped with impurity atoms such as excess $\mathrm{Pb}$, excess I, Te, $\mathrm{Sn}, \mathrm{Bi}, \mathrm{Ge}, \mathrm{La}, \mathrm{Na}$, and $\mathrm{Br}$. In most cases fast (1-2 ns) scintillation was observed at $10 \mathrm{~K}$ and this was greatly reduced when the temperature was raised above $77 \mathrm{~K}$. However, weak scintillation was obtained at room temperature for crystals co-doped with $\mathrm{La}$ and $\mathrm{Br}$ and crystals doped with $\mathrm{Bi}$. We believe that stronger room temperature luminescence will be achievable if the material can be purified. A traveling heating zone furnace has been set-up for purification. Funding from DOE BER to continue this work will be used to prepare ultra high purity $\mathrm{PbI} 2$ in our laboratory using that technique and perfecting the co-doping schemes. We have learned from the LDRD-funded work that the impurities must be controlled to a greater extent than we have been able to do thus far, and that the right impurities in the right concentration are likely to produce the desired ultra-fast scintillator. 
Interaction of Fragile X Mental Retardation Protein with Thymine-DNA Glycosylase: Implication in the Molecular Mechanism of Fragile X Syndrome Principal Investigator(s): Bo Hang

\section{Project Description}

Fragile X Syndrome (FXS), the most common cause of inherited mental retardation, is caused by a CGG repeat expansion in the FMR1 gene followed by hypermethylation in the FMR1 promoter, leading to the silencing of the FMR1 gene and subsequent absence of the fragile $\mathrm{X}$ mental retardation protein (FMRP). During this process promoter hypermethylation is considered to be the most critical event in gene silencing. However, little is known about the molecular mechanism(s) responsible for this epigenetic aberrancy. We have recently found that FMRP associates with and stimulates the activity of human glycosylase TDG. This repair enzyme has been reported to be involved in the process of active DNA demethylation, most likely through its excision activity towards $\mathrm{T} / \mathrm{G}$ mismatches, which can arise from deamination of 5-mC.

The purpose of this project is to investigate whether TDG plays a role in demethylation of the FMR1 promoter and whether the missing FMRP and/or fully expanded CGG repeat in FX cells cause an altered activity or failed targeting of TDG, thus contributing to the maintenance of hypermethylated FMR1 promoter. More generally, these data will aid in understanding cellular mechanisms for transcriptional silencing by active demethylation, which is currently poorly understood.

\section{Accomplishments}

In collaboration with the Priscilla Cooper lab, we have established SV40-transformed FX and control human cell lines and set up a bisulfite sequencing PCR protocol that allows for the analysis of cytosine methylation in the whole FMR1 promoter region of cultured cells.

We then focused on down-regulation of TDG in normal human fibroblasts using siRNA oligomers. Initial data identified an siRNA oligomer that knocks down the TDG expression significantly. Bisulfite sequencing demonstrated a tendency of increase in CpG methylation at the FMR1 promoter. We will use a stable knockdown strategy to further improve and confirm the potential impact of TDG knockdown on promoter methylation in these cells.

We started our work on overexpression of the FMR1 construct in FX cells to examine whether or not hypermethylation at the FMR1 promoter will be reversed. We have cloned the full-size FMR1 gene into a mammalian vector (pcDNA3.1) and are performing the transfection experiments. A rescue effect from overexpressed protein will suggest that FMRP facilitates the expression of its own gene by participating in the active demethylation process.

In the past year, we also discovered a new protein-protein interaction between TDG and XPG. The latter is a key protein in the nucleotide excision repair pathway but with a role in other cellular functions. We are in the process of finding the likely role of this interaction in various cellular processes, such as promoter methylation and the pathogenesis of XP-G/CS. 
Genome Organizer in Carcinogenesis

Principal Investigator: Terumi Kohwi-Shigematsu (P.I.), Mina J. Bissell, Judith Campisi, Yoshinori Kohwi, Sharon Krauss

Project description

The genome organizer SATB1 (Special AT-rich Binding protein) is a key determinant for breast cancer metastasis. Once it is expressed during breast cancer progression, it regulates expression of $\sim 1000$ genes and alters epigenetic status of its direct target genes to promote metastasis. SATB1 expression correlates with poor breast cancer prognosis $(\mathrm{P}<0.0001)$. The purpose of this project is to study regulation of SATB1 in breast cancer cells, how normal and immortalized breast epithelial cells respond to genetically engineered SATB1 expression and mechanisms for these responses, as well as SATB1's role in the cancer cell mitotic machinery. These data will provide a greater understanding of the biogenesis of breast cancer to enable design of therapeutic interventions.

We will employ a series of human breast cancer lines at various stages of disease progression, immortalized non-turmorigenic lines, and normal human epithelial cells to analyze the effect of SATB1 expression on gene expression, signaling events, localization of proteins, and the integrity of cell division machinery. Additionally, we will perform animal model experiments using nude mice. For our investigations we will use state-of-the art biochemistry, molecular biology, and microscopic imaging techniques.

\section{Accomplishments}

The most important accomplishment during the previous year was to initiate studies of effects of SATB1 overexpression on the mitotic apparatus of breast cancer cells. For these purposes we have characterized multiple human breast cancer cell lines which range in aggressiveness and in SATB1 expression. Companion cell lines have been engineered in which SATB1 is either overexpressed or downregulated. Protocols to determine the status of centrosomes and mitotic spindles have also been implemented. Because centrosome amplifications in cancer are highly correlated with genomic instability, our data could critically test the hypothesis that SATB1 expression leads to genomic instability by perturbing mitotic machinery. The results will also document a new role of SATB1 distinct from its role as a genome organizer in the nucleus. In the subsequent year, we will further study the effect of SATB1 expression in the genomic integrity of breast cancer cells and how SATB1 affects mitosis and chromosome segregation.

During the previous year, we have also established an experimental system to alter SATB1 and its target genes in cell culture. We have performed a series of real-time PCR analyses to determine a set of signaling molecules directly regulated by SATB1. Furthermore, we determined that SATB1 expressed in aggressive breast cancer facilitate DNA repair of DNA damage induced by ionizing radiation.

To explore how normal and immortalized cells respond to acquired SATB1 expression, we found that immortalized, but not normal cells, acquired tumorigenicity upon SATB1 expression. However, even between two immortalized breast epithelial cell lines, there is a major difference in their response to SATB1 expression. We are currently analyzing mechanisms underlying these phenotypes since this may have bearing on deciphering multifactorial pathways contributing to the development of breast cancer. 
Expression Profiling of Radiation and Cancer Susceptibility Genes

Principal Investigator(s): Andrew J. Wyrobek and Francesco Marchetti

\section{Project Description}

Our longterm objective is to understand the molecular pathways that determine susceptibility for radiation-induced cancer, specifically breast cancer. It is well known that mammalian tissues differ dramatically in their sensitivity to ionizing radiation and that there are strong genetic determinants of breast cancer risk. In the first phase of this project, we utilized expression microarray and bioinformatics technologies to perform comparative expression profiling of low- and high-dose radiation damage response among tissues of mice including mammary tissue, using strains that differ in their genetic susceptibility to radiationinduced breast cancers. In the next phase of this project, we will investigate the signaling pathways associated with two early proliferative barriers of breast carcinogenesis using the model of human mammary epithelial cells (HMEC) grown in culture. This research will (a) establish the feasibility of using expression profiling and bioinformatics to characterize cell signaling pathways that are modulated in response to ionizing radiation among different tissues, (b) evaluate the concordance in signaling pathways in mammary gland tissues from humans and mice, and (c) provide pilot data of novel molecular mechanisms associated with cellular transitions through two early proliferative barriers to breast cancer.

Accomplishments

Radiation damage response in mouse tissues. We performed high throughput microarray analyses of $\sim 380$ samples of male or female mice of two strains of mice known to differ in their sensitivities for radiation-induced cancers (C57BL/6 and BALB/c). These experiments were designed to characterize relative signaling damage responses of radiation sensitive and resistant tissues to low versus high doses of ionizing radiation. Preliminary analyses identified significant variation among tissues both in the numbers and types of radiation-modulated genes, and analyses are ongoing to identify the signaling pathways associated with tissue-specific damage responses and tissue-variation in cancer risks. Using these pilot data, we obtained new funding from the DOE Low Dose Program to investigate the molecular pathways associated with lowdose radioadaptive response in mammary glands and lymphoid tissues of mice.

Signaling pathways in HMEC. We initiated a collaboration (M. Stampfer, LBL) to investigate the expression profiles in HMECs and paired fibroblasts derived from three unrelated donors using our bioinformatics tools. This study was designed to provide molecular insight into the signaling pathways associated with cellular growth upto and through two early proliferation barriers in human breast carcinogenesis. We have received microarray expression data from $\sim 50$ cell samples at various cell passages before and after the proliferation barriers of stasis which is associated with senescence and agonescence which is associated with telomere dysfunction. Initial bioinformatic analyses identified major differences in expression profiles as cells transition through these barriers (manuscript in preparation). Additional samples are being processed from these three donors to expand the microarray data set to characterize the robust signaling pathways that are associated with cellular transitions through stasis and agonescence barriers. 


\title{
Materials Sciences Division
}

LB06038

\section{Integration of synthetic nano-materials for high speed, robust, and flexible circuitry}

\author{
PI: Ali Javey
}

\section{$\underline{\text { Project Description }}$}

The goal of this is to explore the integration of cheap and high performance electronic circuitry based on synthetic nanostructured channel materials, such as semiconductor nanowires, on bendable and wearable plastic substrates that are capable of operating in the ultra high frequency (UHF) regime (0.3-3 $\mathrm{GHz}$ ). Of particular interest is the development of sensor tapes that can be utilized for biomedical or environmental monitoring applications. A challenge in achieving such sensor tapes is in the placement or deposition of high quality (i.e., crystalline) semiconductors on the bendable substrates. In the past, organic materials have been widely explored for such applications with minimal success, mainly due to the poor performance and low life-time of organic materials as compared to inorganic semiconductors.

Our approach for addressing the above need is to utilize a novel roll printing technology recently developed by our group to assemble parallel arrays of crystalline, semiconductor nanowires on mechanically flexible receiver substrates, such as plastics or papers. These semiconductor nanowires will then be used as the channel material for device and sensor applications. Notably, since the process is performed at ambient temperatures, a multi-step printing process may be envisioned in which arrays of nanowires composed of different semiconductors could be potentially assembled at predefined locations on the substrate and integrated for devices with specific functionalities, such as sensing or electrical switching. In fact, to achieve sensor tapes, both transistor and sensor elements need to be integrated onchip.

\section{Achievements}

We recently reported the large-scale integration of nanowires for heterogeneous, multi-functional circuitry that utilizes both the sensory and electronic functionalities of single crystalline nanomaterials. Highly ordered and parallel arrays of optically active CdSe nanowires and high mobility Ge/Si nanowires are deterministically positioned on substrates, and configured as photodiodes and transistors, respectively. The nanowire sensors and electronic devices are then interfaced to enable an all-nanowire circuitry with on-chip integration, capable of detecting and amplifying an optical signal with high sensitivity and precision. Notably, the process is highly reproducible and scalable with a yield of $\sim 80 \%$ functional circuits, therefore, enabling the fabrication of large arrays (i.e., 13×20) of nanowire photosensor circuitry with image sensing functionality. The ability to interface nanowire sensors with integrated electronics on large scales and with high uniformity presents an important advance toward the integration of nanomaterials for sensor applications. 
Transport in Thin Polymer Films

PI: Blandine Jérôme

Project description

The purpose of this project is to study the transport properties of confined polymeric systems, with a special focus on the anisotropy of the diffusion through confined systems, and the effect of the conformation of polymer chains on transport. This requires developing Resonance Soft Xray Reflectivity to obtain the necessary orientational information on polymer chain conformation.

Our approach consists in studying in parallel the structure of confined polymers (developing and exploiting new possibilities offered by Resonant Soft X-ray reflectivity) and the resulting transport properties obtained through dynamic mass up-take measurements with a quartz microbalance. Uniform films of homopolymers and nanostructured films of mixtures of homopolymers blended by block copolymers are used to tune the sample geometry in which transport is measured.

\section{Accomplishments}

We have concentrated on getting some of the missing information concerning the structure of polymers under confinement. One property for which very little is known is the distribution of orientation of the segments of chains with respect to the normal to the film surfaces. Surfaceinduced alignment could have a strong influence on the diffusion through confined polymers, as it is likely to increase the diffusion parallel to the surfaces and decrease it perpendicular to the surface.

In close collaboration with J.B. Kortright of the Materials Sciences Division, we have started developing the use of Resonance Soft X-ray Reflectivity (RSoXR) to provide the profile of orientational order of polymer chain segments in thin films. As first step in this development, we have obtained the first evidence of confinement-induced chain alignment in ultra-thin polyisoprene films, by measuring the resonance absorption of soft X-rays with different polarizations at the carbon K-edge (measurements performed the the Advanced Light Source at LBNL).

In parallel to these structural studies, we have set up absorption and diffusion measurements in thin films using a quartz microbalance in an environmental chamber. By depositing films on the resonator of a quartz resonator, putting it in the presence of a vapor or liquid of defined composition, and following the mass up-take in time through the resonance frequency of the quartz resonator, we are now able to measure both the solubility and the diffusion of the molecules present in the chamber through the polymer film. Our first case study concerns the behavior of amorphous polylactic acid films in the presence of water vapor. 


\section{Conducting Metal-Organic Frameworks \\ Principal Investigator: Jeffrey R. Long}

\section{Project Description}

This project aims to induce electronic and ionic conductivity in porous metal-organic frameworks. While metal-os have shown great potential for their applications in the adsorption of small molecules, few studies report electronic or ionic conductivity in such porous compounds. To induce electronic conductivity, we will develop new frameworks with select metal cations and ligands to enhance electron transfer throughout the framework. Work will focus on frameworks based on octahedral $\mathrm{Fe}^{\mathrm{II} / I I I}, \mathrm{Ru}^{\mathrm{I} / \mathrm{III}}, \mathrm{Os}^{\mathrm{II} / \mathrm{III}}$, and $\mathrm{Mo}^{0 / \mathrm{I}}$ redox centers which have demonstrated electron delocalization in discrete complexes. These cations will be linked into a porous framework using nitrogen and sulfur-based ligands to enhance metal-ligand bond overlap, in contrast to the use of carboxylic acid ligands observed in many reported frameworks.

We also plan to examine the relationship between pore size/structure and lithium ion conductivity in a host of known MOFs. Anionic frameworks containing exchangeable cations will be impregnated with lithium cations, and neutral frameworks will be impregnated by lithium cations and small anions.

\section{Accomplishments}

To this date, our work has focused predominantly on the pursuit of electronic conductivity. As an initial study of an electronically conducting framework, we have prepared a number of $\mathrm{Fe} / \mathrm{Ru}$-based Prussian blue compounds. Though the asymmetric environment caused by the cyanide ligand prevents extensive delocalization, the conductivity of the original $\mathrm{Fe}_{4}\left[\mathrm{Fe}(\mathrm{CN})_{6}\right]_{3} \cdot 18 \mathrm{H}_{2} \mathrm{O}\left(5.5 \times 10^{-5} \mathrm{~S} / \mathrm{cm}\right.$, ambient temperature $)$ has been somewhat improved by two orders of magnitude for $\mathrm{K}_{1.2} \mathrm{Ru}_{3.6}\left[\mathrm{Ru}(\mathrm{CN})_{6}\right]_{3} \cdot 16 \mathrm{H}_{2} \mathrm{O}\left(5.7 \times 10^{-2} \mathrm{~S} / \mathrm{cm}\right)$. This increase in conductivity is also mirrored by a shift in the intervalence charge transfer band to lower energies. Having successfully characterized the degree of electron delocalization using temperaturedependent conductivity measurements and optical spectroscopy, and we are now confident in the application of these techniques to future materials that we prepare. A complication has been the presence of loosely coordinated alkali cations and water, which may contribute to a small ionic component in the total conductivity. In our future work, more rigorous DC/AC measurements will help differentiate these two mechanisms.

We have also examined conductivity in the 1-D chains of $\mathrm{Ru}_{2}(\mathrm{OAc})_{4} \mathrm{pz}(\mathrm{pz}=$ pyrazine) and a previously reported framework based on the mixed-valent $\mathrm{Fe}_{3} \mathrm{O}(\mathrm{OAc})_{6}$ motif. The electronic conductivities of both materials still remain fairly low, however. To address this, further work will focus on sulfur-based ligands to increase orbital overlap between the metal and ligand. We have prepared some dithiocarboxylate ligands together with paddle-wheel complexes of $\mathrm{Fe}^{\mathrm{II} / \mathrm{III}}$ and $\mathrm{Ru}^{\mathrm{II} / \mathrm{III}}$, and are now ready to investigate the synthesis of extended frameworks based on the reaction between these two precursors. 


\section{Fracture of Cortical Bone: Mechanisms Underlying the Origins of Toughness in Hard Mineralized Tissues \\ Principal Investigator: Robert O. Ritchie}

\section{Project Description}

The intent of this program was to provide a fundamental understanding of why human bone fractures and the mechanisms by which this biological material, and other hard mineralized tissues such as dentin, develop their resistance to cracking. Our objective was both to understand natural toughening mechanisms that could be applied in the design of new non-biological, light-weight high-toughness synthetic materials, and to examine how biological factors, such as age, osteoporosis and therapeutic treatments, affect the structural properties of bone, and how this translates to the well known increased risk of bone fracture in the aged. The work essentially was aimed bridging the gap between the existing clinical understanding of bone fracture and the underlying micromechanical mechanisms of bone failure. The approach was distinctly materials science based and employed quantitative methods that span the relevant hierarchical length scales associated with fracture in bone, with particular emphasis on in situ studies of fracture. In addition, measurements at the nano/molecular scales (pico-force AFM, nanoindentation, and vibrational spectroscopies) and microscale (in situ electron microscopy, computed hard and soft x-ray tomography) were related to structural behavior at the macroscale.

\section{Accomplishments}

Using new in situ fracture testing methods developed in our laboratory, we discovered that the fracture toughness of human cortical bone at physiologically relevant crack sizes is actually far higher than previously reported in the transverse (breaking) orientation, and lower in the longitudinal (splitting) orientation. We were able to relate such information to the salient toughening mechanisms in bone which originate with microcracking phenomena primarily along the cement lines (i.e.,the interfaces of the secondary osteons); such microcracking results in macroscopic crack deflection and significant toughening for transverse fracture and crack bridging by "uncracked ligaments" for longitudinal fracture (Fig. 1). We also worked with mouse and rat animal models to define best practices for assessing the strength and toughness of small animal bones, and to examine the role of glucocorticoids in degrading toughness and bisphosphonate treatments in preventing such degradation. This work has demonstrated that a rigorous evaluation of the mechanical performance of bone with an emphasis on the underlying nano/microstructural mechanism is not only possible, but is required to address the public health challenges of increasing bone fracture rates in the elderly.
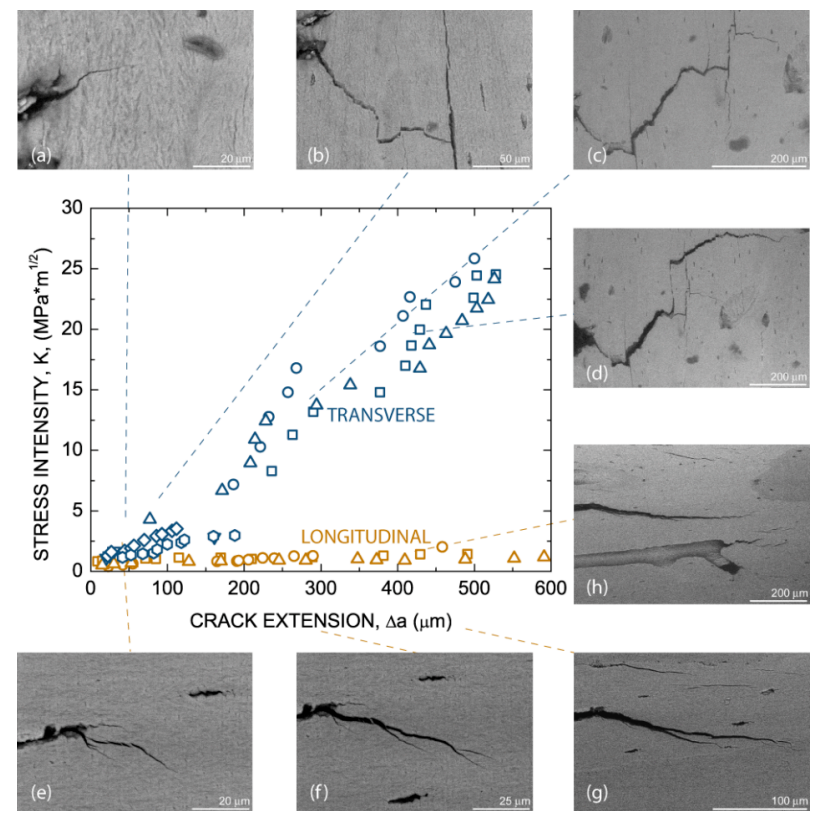

Figure 1. Fracture-toughness R-curves for the transverse and longitudinal orientations in human cortical bone in $37^{\circ} \mathrm{C}$ Hanks' Balanced Salt Solution, with simultaneous environmental scanning electron microscope imaging of the crack paths, showing the dramatically higher toughness of bone in the transverse orientation associated with gross crack deflection. Crackdriving forces are presented in terms of the equivalent stress-intensity, calculated from nonlinear-elastic measurements of the $J$ integral, using both elastic and plastic components. 
Three dimensional printing of ceramics with submicron resolution Principal Investigator(s): Eduardo Saiz

\section{Project description}

New techniques for the fabrication of complex three dimensional (3-D) structures are urgently needed for a variety of a pplications such as light-weight s tructural $\mathrm{m}$ aterials, micro-fuel cells, batteries, chem ical sensors, filtration membranes for molten metals and hot gases, bioreactors, catalyst carriers, electrodes for batter ies and solid oxide fuel cells, insulators, photonic crystals or tissue engineering, to nam e a few. For many of those it will be highly advantageous if the complex shape is combined with large porosity at the micro and nano-levels. The advent of solid-fre e-form or direct pr inting technologies has opened the possibility of building these struct ures following intric ate computer designs. The persistent challenge is to develop novel fa milies of inks and approaches to print materials with a wide range of chemistries and increased precision and reproducibility.

The main goal of this proposal is to formulate new inks for the direct printing of three dimensional ceram ic-based com posites with submicron resolution. These technologies will be app lied to the $\mathrm{f}$ abrication of new $\mathrm{f}$ amilies of com plex hie rarchical hy brid structures with arch itectures simultaneously controlled at $\mathrm{m}$ ultiple dimensions from the nano to the macroscopic scales.

\section{Accomplishments}

The main accomplishment of the $p$ roject has $b$ een the formulations o f new inks to print high ly porous ceram ic and $\mathrm{m}$ etal-ceramic composites with chem istries and porosities controlled from the nano-level up. The process is based on a sol-gel approach that uses th e accu rate contro $l$ of the nucl eation and $g$ rowth of a $f$ ugitive pha se to manipulate the pore size and shape. This phase (for example amm onium chloride) is subsequently eliminated through therm al treatments and/or dissolution to leave a highly porous ceramic skeleton.

The starting solutions are com bined with water based hydrogels to create inks with the right rheological properties to pr int complex three dim ensional shapes using robotic assisted deposition. The inks flow through narrow nozzles allowing printing with highprecision. The use of a sol-gel based approach enables the fabrication of materials with a wide range of chem istries. W e have synt hesized highly porous ( $\mathrm{p}$ orosity $>90 \mathrm{vol} \%$ ) ceramic (e.g. $\mathrm{Al}_{2} \mathrm{O}_{3}$ or $\mathrm{ZrO}_{2}$ ) and metal-ceramic nanocomposites (e.g. $\mathrm{Ni}-\mathrm{Al}_{2} \mathrm{O}_{3}$ ). We are currently working in modeling the rheological properties of these complex inks such that their composition can be refined in order to increase the printing reso lution. In the near future we plan to use this technology to fabricate $m$ aterials optim ized for specific applications from catalysis to tissue engineering. 
Quantifying the Quantum Backaction of a Non-Linear Dispersive Measurement Principal Investigator: Irfan Siddiqi

\section{Project Description}

A canonical m ethod to read out a quantum spin system is to couple it to an oscillator. This technique, first developed in atom ic physics, is known as a dispersive $m$ easurement. When the quan tum spin inte racts with the oscillator, its ef fect is to modify the oscillator frequency. Thus the oscillator has two di fferent resonant frequencies co rresponding to the up and down states of the spin. One can determine the frequency of the readout oscillator by energizing it with a $\mathrm{m}$ icrowave frequency electrom agnetic pu lse and observing the phase shift in the resulting reflected or transmitted signal.

In quantum mechanics, any act of $\mathrm{m}$ easurement, even this seem ingly benign dispersive one, perturbs the system being $\mathrm{m}$ easured. In this case, the energy splittin $\mathrm{g}$ of the spi $\mathrm{n}$ systemthe Larm or frequency-becom es shifted when the oscillator is energized. This is known in atomic physics as the AC Stark effect. If the osci llator is energized with a larger num ber of photons $\mathrm{N}$ in the $\mathrm{m}$ easurement pulse, the Stark sh ift is enhanced and the dephasing becom es greater. At the sam e tim e, the $m$ easurement has a greater signal to $n$ oise ra tio with a la rger photon field and the state can be determ ined in a shorter tim e. One c an show that there is an uncertainty relation between the measurem ent time and the dephasing rate, which is sim ply a restatement of Heisenberg's uncertainly prin ciple. Furthermore, it can ri gorously be proven that for a harmonic oscillator, the dephasing is mini mal, and can be ch aracterized as "quantu $\mathrm{m}$ limited". Are th ere o ther sch emes which a lso obey this type of $m$ easurement uncertainty relation? One natural question that com es to mind is whether a non-linear os cillator also results in minimal dephasing. What is intriguing about th is question is that a nonlinear system can be viewed as a $\mathrm{m}$ edium to provide ga in - that is, it is an am plifier. Thus, this ques tion is re ally asking: "What is the price to pay for amplifying quantum information?"

Accomplishments

We have equipped our dilution $r$ efrigerator will fully filtered measurement lines for 1-2 $\mathrm{GHz}$ operation at $10 \mathrm{mK}$. The $\mathrm{m}$ icrowave circu it, inc luding a custom ized low tem perature amplifier, is functional and has the expected gain and noise temperature. We have also installed a new type of cryogenic s witch which will allow us to "hot s wap" between different am plifiers to compare their quantum backaction. The switch is functional at $10 \mathrm{mK}$.

We have successfully fabricated ch arge-phase qubits and have started $\mathrm{m}$ easurements of their coherence tim es. Our first samples have exhibited a working readout oscillator and a working two level system. W e have perform ed spectroscopy of the qubit and observe correct variations with bias charge and flux.

Our goal now is to maximize qubit coherence by tuning the fabrication parameters and to then study the coherence properties as a functi on of the num ber of photons in the readout resonator. 


\section{Two-element ultracold atom simulator of materials Principal Investigator: Dan Stamper-Kurn}

\section{Project Description}

The long-term goal of this LDRD-sponsored project is to achieve physical realizations of models in condensed-matter physics, e.g. spin-lattice models or models of quantum magnetism with itinerant electrons, using ultracold atoms. Such a "quantum simulator" will use gases of ultracold rubidium and lithium atoms that are both held in the spatially periodic potential created by intersecting off-resonant laser beams, and that interact by tunable contact interactions.

Over the one-year duration of this project, we undertook to develop the experimental infrastructure to achieve this admixture of ultracold atomic gases. Such infrastructure includes the development of laser system, a two-atomic-species beam source, and a high-resolution imaging system suitable for both species. We sought also to develop further insights on magnetism in quantum gases so as to guide upcoming research.

\section{Accomplishments}

We report four accomplishments at the conclusion of this project:

1. We studied the evolution of helical spin textures in a rubidium spin-1 spinor Bose-Einstein condensate, produced by a sequence of rf pulses and pulsed magnetic field gradients. Following its preparation, the texture was allowed to evolve freely in a homogeneous magnetic field. The longwavelength texture was found to break up and produce a short-wavelength pattern of spin domains. We confirmed the ascription of this behavior to magnetic dipolar interactions by varying such interactions using NMR-inspired rf-pulse techniques. These experiments provide surprising and convincing proof that magnetic dipole interactions are an essential influence on the spin-1 rubidium gas.

2. Given the identification of the spin-1 rubidium quantum gas as an essentially dipolar magnetic quantum fluid, we addressed the pressing question of what is its equilibrium phase. For this, we cooled a non-degenerate and unpolarized spin-1 gas to the regime of quantum degeneracy, and discovered that the low-temperature phase of this gas is a spin-domain crystal. This discovery raises the tantalizing possibility that the spin-1 Rb gas may be a supersolid. To test for this possibility, we are undertaking experiments to measure the phase coherence properties of this gaseous crystal.

3. Using a microwave system developed earlier, we examined further the dynamics of spontaneous magnetization in the spin-1 Rb gas. We established that spin domains formed in a gas quenched across a quantum phase transition are likely produced by the quantum amplification of quantum spin fluctuations. The dynamical instabilities probed in this manner may find application as quantum-limited amplifiers of spin, e.g. in a magnetic-field sensor. Theoretical work, undertaken in collaboration with LBNL scientist Prof. Marvin Cohen and his group, explored the influence of dipolar interactions on the domain formation process and also the possibility of producing spin-squeezed gases.

4. Infrastructure for a two-element quantum simulator was constructed and tested, including a two-element atomic beam source, a two-element Zeeman slower, a UHV vacuum chamber in which the quantum simulations will be performed, a magnetic conveyance system to deliver ultracold gases from the magneto-optical trapping region to the lattice-trapping region, and a long-working-distance microscope that achieves $\sim 1$ micron diffraction-limited resolution. 


\title{
Understanding Electronic Energy Level Alignments at Nanoscale Interfaces
}

\author{
Principal Investigators: Jeff Urban, Taleb Mokari, Delia Milliron, Jeff Neaton, David \\ Prendergast
}

Project Description: Predicting and measuring the electronic energy levels, gaps, and alignments in nanoscale systems as done for bulk materials, is currently impossible. We propose to develop novel heterostructured nanomaterials that will contribute tremendously to solve fundamental problems in solar energy while also addressing major scientific questions such as: What physics controls electronic level alignment at nanoscale interfaces? In terms of systems design, can the electronic structure of a composite nanostructure be understood in terms of the bulk properties of its constituent materials? How can we think about "semiconductor" interfaces when the system size is far less than typical depletion lengths? Are the terms "Schottky" and "Ohmic" even meaningful at the nanoscale? How do internal fields associated with change transfer impact electronic level alignment?

Our effort interweaves frontiers in heterostructured materials, instrumentation, and firstprinciples theory here at LBNL. Specifically, we are currently collaborating on measuring, calculating, and understanding electronic and vibrational energy levels in novel multi-component nanoscale heterostructures, with a focus on: (a) two distinct metal-semiconductor nano-interfaces; (b) a single, finite semiconductor heterojunction; and (c) different classes of organic-inorganic interfaces (specifically, the interfaces between the capping ligands and the metals and semiconductors, respectively). Completion of this study will forge new paths in rational nanoscale materials design.

Accomplishments: Since the project initiated this summer, we have already made significant progress toward our goals. We have synthesized, characterized, and begun to analyze nanowires with semiconductor-semiconductor (CdSe-CdTe) and semiconductor-metal interfaces (CdSe-Au). We have also purchased, installed, and optimized a scanning near-field optical microscope (SNOM); this equipment will enable us to measure the vibrational and electronic interfaces of these materials with sub-wavelength resolution. The theoretical component of this effort has already calculated how bound ligand molecules modify the strain at the surface of the semiconductor wires and also the vibrational energies of the ligands themselves. We have recently begun to measure the vibrational characteristics of the capping groups (comparing the vibrational modes of bulk powders to nanowires-bound moieties) and are currently mapping out a realistic model of the surface of a semiconductor nanowire, a full experimental picture which accounts for surface strain, ligand coverage, and modifications to surface energies. We are in the process of completing this study and plan to move forward to study how these surface modifications contribute to changes in the electronic structure of the nanowires systems. 
Transition-Metal-Doped GeTe Nanowires as a Single-Phase Multiferroic System

$$
\text { Principal Investigator(s): Junqiao Wu }
$$

\section{Project Description}

We proposed to explore the possibility of establishing magnetoelectric multiferroics in a single-phase system of transition metal doped GeTe nanowires. GeTe has long been recognized as a non-volatile data storage material which takes advantage of its phase change upon the application of an optical pulse. Less explored is the GeTe ferroelectric (FE) transition which occurs at a Curie temperature of approximately $650 \mathrm{~K}$. Substitution of Ge by transition metal elements in bulk GeTe reduces the FE Curie temperature and, in some cases, introduces carriermediated ferromagnetism (FM) with a Curie temperature of up to $140 \mathrm{~K}$. The possible coexistence of FM and FE in this single-phase, semiconducting material establishes a versatile multifunctional system with a new type of multiferroic.

In this project we will synthesize GeTe nanowires using the vapor transport method, providing a quasi-1D model system for exploring the possible multiferroics and its interplay with the phase change characteristics. As an initial probe of their nanoscale properties, we will use in situ TEM to analyze the melting, evaporation and sublimation behavior and thermal stability of the nanowires under high vacuum. Possible size and surface effects in the phase diagram of GeTe will be investigated and characterized. These results will lay the foundation for synthesizing doped GeTe nanowires and understanding their phase transition physics.

\section{Accomplishments}

Our most significant accomplishment is to in situ observe and quantify the congruent sublimation of GeTe nanowires for the first time. GeTe nanowires with diameters varying from 20 - $200 \mathrm{~nm}$ were grown by the vapor transport method using Au nanoparticles as catalysts. We have studied the solid-liquid-vapor phase transition of GeTe nanowires in a TEM equipped with a heating stage. The geometric constraints in nanowires allowed the in situ investigation of their melting, evaporation, and decomposition with high spatial, structural, and compositional resolution. The dynamic monitoring enabled by the in situ experiments was used to image and track the evolution of the phase interface. Our experiments show a strong suppression in melting point compared to bulk, and congruent GeTe evaporation or sublimation depending on local pressure and temperature. These results allow us to evaluate the phase transition enthalpy and vapor pressure of this system at the nanoscale.

Related to the phase change property of GeTe and resultant resistance switching, we have also characterized a non-volatile resistance switching in planar $\mathrm{NiO}_{\mathrm{x}}$ devices, and directly observed nanoscale filaments that are responsible for the switching.

We are currently in the process of applying the developed in situ TEM technique to various oxide nanostructures that exhibit a variety of phase transitions as a function of external stimuli such as temperature, stress and electric field. 
Hierarchical Assemblies Of Peptide-Polymer Conjugates

Principal Investigator(s): Ting Xu

\section{Project Description}

De novo designed peptides have a chemical and structural diversity that is comparable to or greater than that found in natural materials, yet they are typically more robust. However, they are limited by stability and degradation encountered during handling and are not amenable to standard fabrication processes. The purpose of this project is to study the principles governing the hierarchical self-assembly of complex systems involving designed peptides and polymers and use the knowledge to generate tailored functional materials. The proposed cross-disciplinary effort integrates the ease of processing and self-assembling of synthetic polymers on the tens to hundreds of nanometer level with the unique molecularly driven assemblies and functionalities of peptides and proteins. We mainly focused on fundamentally understanding the interactions between each building block and delicately balancing and manipulating these interactions to achieve targeted assemblages without interfering with the peptide secondary structures and designed functionalities. The functional soft materials resulting from the proposed study will have applications in selective separation for drugs and biofuels, high efficiency organic photovoltaic fabrication, as well as, information storage and communication devices and will have significant potential to impact human health, environment and renewable energy.

Accomplishment

Our most significant accomplishment is to develop a new design of peptide-polymer conjugates where a polymer chain is covalently linked to the side chain of a helical bundle-forming peptide. The effect of conjugated polymer chains on the peptide structure was examined using a de novo designed 3-helix bundle and a photoactive 4-helix bundle. Upon attachment of poly(ethylene glycol) to the exterior of the coiled-coil helical bundle, the peptide secondary structure was stabilized and the tertiary structure, i.e. the coiled-coil helical bundle, was retained. Using a hemebinding peptide as an example, the new peptide-polymer conjugate architecture also preserves the built-in functionalities within the interior of the helix bundle.

It is expected that the conjugated polymer chains act to mediate the interactions between the helix bundle and its external environment. Thus, this new peptide-polymer conjugate design strategy may open new avenues to macroscopically assemble the helix bundles and may enable them to function in non-biological environments. We are in the process of studying the co-assembly of helix bundle forming peptide-polymer conjugate and diblock copolymer in thin films. Preliminary results suggested that peptide-polymer conjugates could be patterned on a nanoscopically patterned surface in a controlled manner. 


\section{Nuclear Science Division}

New Experimental Program to Deduce $(n, f)$ Cross-Sections for Advanced Fuel Cycle Studies

Roderick Clark

Project description:

This is the second year of a new research effort to determine basic nuclear physics data for Advanced Fuel Cycle (AFC) studies. Sustainable, safe nuclear energy has been identified as an important national need and the necessary basic research is part of the DOE mission. We are attempting to determine cross-sections for neutron-induced fission of actinides involved in fast reactor fuel cycles. Existing data often show significant discrepancies and do not extend to the higher neutron-energy range (up to $\sim 20 \mathrm{MeV}$ ) found in fast reactors. The goal is to develop novel surrogate reactions to deduce the relevant cross-sections to unprecedented accuracy $(<10 \%)$ over a broad neutron-energy range $(0.2$ to $20 \mathrm{MeV})$. The surrogate reaction method uses a light-ion reaction to determine the decay probabilities of a compound nucleus of interest. The formation probability can be modeled accurately. By combining the calculated formation probability with the measured fission probability the desired (n,f) cross-section can be deduced. We use light-ion beams, accelerated by the 88-Inch Cyclotron, which are incident on actinide targets. Charged particles and fission fragments are detected in the STARS Si-detector array in order to determine the required fission probabilities.

Accomplishments:

Over the last year a strong LBNL/LLNL collaboration has studied various aspects of surrogate reactions. Researchers from PNNL, UC Berkeley, and the University of Richmond have also joined the collaboration. Experiments using several different surrogate reactions have been performed at the 88-Inch Cyclotron using the STARS+LIBERACE array, including:

- The ${ }^{235} \mathrm{U}(\mathrm{d}, \mathrm{pf})$ reaction was used as a surrogate for the ${ }^{235} \mathrm{U}(\mathrm{n}, \mathrm{f})$ reaction. This has a well known cross section and the experiment aimed at benchmarking the utility of surrogate reactions induced with deuteron beams

- ${ }^{3} \mathrm{He}$-induced reactions on ${ }^{232} \mathrm{Th}$ and ${ }^{236} \mathrm{U}$ targets were used to investigate the $\left({ }^{3} \mathrm{He}, \mathrm{p} / \mathrm{d} / \mathrm{t}\right)$ channels as surrogates (both in absolute and relative measurements). The results will have particular relevance to studies of the Th cycle.

- ${ }^{18} \mathrm{O}$-induced reactions were used to investigate the possibility of using heavier ions in transfer reactions such as $\left({ }^{18} \mathrm{O},{ }^{16} \mathrm{O}\right)$ and $\left({ }^{18} \mathrm{O},{ }^{20} \mathrm{Ne}\right)$ as surrogates.

In addition, analysis of data from prior experiments has continued. LBNL scientists have analysed data on the ${ }^{238} \mathrm{U}\left({ }^{3} \mathrm{He}\right.$, tf $)$ reaction, an absolute surrogate measurement for determining the ${ }^{237} \mathrm{~Np}(\mathrm{n}, \mathrm{f})$ cross section, and have identified important issues related to proper efficiency determination in the experimental set-up. Benchmarking results on $(\alpha$, $\left.\alpha^{\prime} f\right)$ and $\left({ }^{3} \mathrm{He}, \alpha f\right)$ surrogate reactions on $U$ targets have been published. Several more publications are expected soon. 


\section{Development of a $100 \mathrm{~km}^{3}$ Neutrino Detector for Ultra High Energy Neutrinos} Principal Investigator: Spencer Klein

\section{Project Description}

We are developing the physics case and detailed design for a $100 \mathrm{~km}^{3}$ neutrino detector to detect ultra-high energy (above $10^{17} \mathrm{eV}$ ) neutrinos. These neutrinos are produced by 'GZK interactions' between the highest energy (above $4 * 10^{19} \mathrm{eV}$ ) cosmic rays and photons from the $3^{0} \mathrm{~K}$ cosmic microwave background radiation. A substantial fraction of the detected neutrinos will come from cosmological distances, allowing us to probe the evolution of high-energy accelerators in the universe. At the same time, we will search for point sources of these neutrinos. To do this, we need to detect of order 100 events in a few years; this requires a detector volume of order $100 \mathrm{~km}^{3}$; this effort will require a large collaboration.

We will detect coherent radio Cherenkov radiation or acoustic pulses from neutrino-induced showers in Antarctic ice. Both of these signals propagate long distances $(\sim \mathrm{km})$ in Antarctic ice, so one can build a very large detector with a reasonable number of sensors.

\section{Accomplishments}

Our first effort was to examine the existing radio and acoustic efforts. The South Pole acoustic detectors require drilling fairly deep (500-1000 m), and correspondingly expensive holes. The South Pole radio efforts also require holes, but these can be shallower (50-200 m deep). This drilling is cheaper, but still a significant cost factor.

One new and very promising approach is to build a radio-detection array on the Antarctic coast, about $110 \mathrm{~km}$ from McMurdo Station. The ice-water interface below the $625 \mathrm{~m}$ thick Ross Ice Shelf is an excellent radio mirror, reflecting downward-going Cherenkov radiation to the surface. Since the earth absorbs high-energy neutrinos, most signal events are downward or horizontal, and this reflection leads to a large increase in effective volume. The Ross Ice Shelf is more uniform than the South Pole ice, so no drilling is required. Measurements have shown that the site is largely free of anthropogenic radio background, and logistics support is available from McMurdo station.

This approach is being pursued by the nascent ARIANNA collaboration. ARIANNA will deploy radio-detector stations on a $~ 300 \mathrm{~m}$ to $1 \mathrm{~km}$ grid. Each station will have an octagonal array of eight downward-pointing antennae emplaced in slits cut in the ice. ARIANNA requires a data acquisition system able to sample antenna waveforms at speeds up to 2 gigasamples/s, with low-noise and low-power. The system must operate very reliably over a very wide temperature range. If ARIANNA is funded, this would be an LBNL responsibility. With the collaboration coming together, we are building prototypes of the key pieces of this system, starting with the low noise preamplifiers and testing commercial building blocks, such as GPS receivers.

We are also working on improved simulations of the expected signals - radio Cherenkov radiation from electromagnetic showers produced by neutrino interactions. Our current focus is on a more accurate evaluation of the electromagnetic cross-sections at high energies, where bremsstrahlung and pair production can no longer be described as point interactions. These cross-sections will be used in new ARIANNA simulations. 


\section{Physics Detector and Sensor Technologies Applied to Geological and Geophysical Applications at DUSEL - Kevin Lesko}

Project Description:

The purpose of this project is to develop solutions to R\&D issues, including significant societal problems, utilizing the new opportunities provided by the Deep Underground Scientific and Engineering Laboratory (DUSEL). Partnerships between Earth Scientists, Engineers and Physicists will be developed to investigate underground carbon remediation techniques, and develop novel detector components and materials for underground research. The first activity researches underground carbon sequestration, developing techniques for transporting, and monitoring $\mathrm{CO}_{2}$ and understanding long term sequestration by characterizing the site and developing monitoring tools and techniques. The second topic will investigate detector materials with a focus on ultra-low contamination of radioactive species and novel detector designs to mitigate and minimize the negative impact of detector contamination.

We draw on Earth Sciences experience in characterizing the underground, coupled processes with $\mathrm{CO}_{2}$ sequestration and Physics instrumentation, Monte Carlo simulations, and sensors. The multidivisional team, together with established DUSEL collaborations, work together to quantify the distribution of radioactivity in the rock, correlate the heat flows with crustal $U$ and Th, construct models, and evaluate the leakage and storage of gaseous and liquid fluids. DUSEL test sites will be located to conduct in situ experiments for fluid flow, chemical transport, and heat transfer, measure the migration and leakage of injected $\mathrm{CO}_{2}$. We draw on physicists and engineers, in particular the Germanium detector lab to develop assay techniques, ultra-clean materials, and novel detector designs.

Accomplishments:

Carbon Sequestration: using the located test sites along sandline boreholes in Homestake we began developing procedures and techniques to characterize the sequestration test bed and to monitor the evolution of injected $\mathrm{CO}_{2}$, including phase transition, migration and dispersion. We have assessed the current, national, $\mathrm{CO}_{2}$ sequestration demonstration projects and likely large-scale sequestration repositories. From these projects we have identified on key research foci for the instrumentation including determining leakage, and migration within underground repositories. Assessing well caps and borehole casing have been noted in particular, as needing for detailed monitoring. The insertion of supercritical $\mathrm{CO}_{2}$ is being pursued as the preferred form for introducing carbon in the underground. The phase transitions and migration of supercritical $\mathrm{CO}_{2}$ in the most promising repository is a complex interaction. We have developed plans to assemble large-scale research project to observe and document the interactions of the $\mathrm{CO}_{2}$ with the repository media. We will pursue sensors, working towards the integration of a new family of gas micro-sensors to observe and document the leakage of $\mathrm{CO}_{2}$ from the well caps and borehole casings. Further we will pursue instrumentation and experimental arrangements to inject and monitor supercritical $\mathrm{CO}_{2}$ including plume formation and mixing.

New Materials and Sensors: Next generation detectors require special materials including ultra-low background materials, ultra compact and ultra-pure electronics for detectors, and remote sensor arrays. We research recent developments and identify candidate technologies for ultrapure materials and producing detectors from these materials. Particular promise in a low capacitance, high bandwidth solidstate detector design has been demonstrated this year. Starting with a commercially supplied detector blank, we manufactured this novel detector design and have characterized it behavior and performance. We have initiated additional research with this detector to introduce additional electrical contacts and a higher level of segmentation. This higher segmentation introduces additional timing and a degree of event reconstruction within the detector. Additional detector blanks have been acquired and point-contact detectors are being created from these. We will pursue additional contact technology investigations with these prototypes to refine the long-term characteristics of the detector design. This point-contact detector is a likely potential for deployment in DUSEL for sensitive measurements with ultra-low background requirements. 
Hyperons in Polarized Proton Collisions and the Origin of the Nucleon Spin Principal Investigator(s): Ernst Sichtermann

\section{Project Description}

The goal of the project is to advance the understanding of the nucleon spin composition in terms of its constituent quarks and gluons. The combined contribution from quark and antiquark spins has been measured and is remarkably small, in striking contrast with expectations based on models that enjoyed success in describing hadron spectroscopy and magnetic moments. This project aims to delineate the quark and anti-quark spin contributions to the nucleon spin by flavor by determining the longitudinal spin transfer of hyperons and anti-hyperons produced with large transverse momenta in collisions of polarized proton beams at the Relativistic Heavy Ion Collider (RHIC). This gives the STAR experiment at RHIC unique access to the spin distributions of strange quark and anti-quarks in the polarized proton and, in principle, allows also a novel way to access the spin distributions of the up and down quarks and anti-quarks.

The project required the development of the phenomenology of (anti-)hyperon polarization in polarized proton collisions, a proof-of-principle spin analysis of hyperon data collected with the STAR experiment at RHIC, and the development of an experimental trigger concept to efficiently collect data on (anti-)hyperons with high transverse momenta.

\section{Accomplishments}

We have systematically mapped the phenomenology of the Lambda, Sigma, and Cascade anti-hyperons. The Sigma hyperon was found sensitive to the spin distributions of the up and down anti-quarks, and the Cascade to that of the strange anti-quarks. The results have been published in Physical Review D 78, 054007 (2008).

We have completed a proof-of-principle analysis of longitudinal spin transfer for Lambda and anti-Lambda from data collected with the STAR experiment at RHIC in the year 2005 using a beam-collision trigger to start the experiment read-out. A control sample of the more abundantly produced and spinless K-mesons was used to assess systematic uncertainties in the measurement and these uncertainties are under control to sufficient precision. The preliminary results from these studies were presented at the SPIN-2006 conference and are published in the conference proceedings.

We have analyzed STAR data obtained with more advanced experiment triggers, namely a calorimetric jet-patch trigger and a high-tower trigger. The jet-patch trigger starts the experiment read-out when a large energy flow, a jet, is observed as an energy deposit in an extended area of the STAR barrel electromagnetic calorimeter. The data thus obtained extend to considerably higher transverse hyperon momenta than data obtained with a beam-collision trigger, albeit with additional systematic uncertainties. Detailed further study showed that a significant fraction of the anti-protons from anti-hyperon decay convert in the STAR barrel electromagnetic calorimeter towers. The associated energy deposit is sufficiently large to satisfy a high-tower trigger condition. We have identified the high-tower trigger in combination with spatial information from a time-of-flight detector, an ongoing STAR upgrade, as a viable path for future (anti-)hyperon spin measurements. The present results have been prepared for publication in Physical Review D and are currently under review within the STAR collaboration. 
Development of a Low-Energy, High-Current Astrophysics Accelerator

Principal Investigators: Paul Vetter, Daniela Leitner, Matthaeus Leitner, Damon Todd

\section{Project Description}

We seek to develop an ion accelerator facility with which to measure astrophysical nuclear reaction cross sections. Precise determinations of many cross sections are needed to inform nearly every area of nuclear astrophysics. Nuclear astrophysics seeks to explain the origin of elements in stars and stellar explosions; to understand stellar and galactic evolution (the timescales of stellar burning phases and explosive events, and changes in galactic star population and the isotopic composition of solar system and galactic material), and to interpret current and planned solar neutrino oscillation experiments. A major challenge to these goals is the lack of high precision data for certain hydrogen burning, helium burning, and neutron producing reactions. To measure these reaction cross sections, the beam energy must generally be as low as possible to mimic stellar burning conditions and to measure near-threshold resonant nuclear contributions. Below $100 \mathrm{keV}$, measuring the low event rates requires intense beams $(>100$ $\mathrm{mA}$ ), high density targets, high-efficiency event counting, and good background rejection. We are designing a dual accelerator facility including an ion transport system, high-flow gas jet target, target region, and detector system to meet these requirements.

\section{Accomplishments}

We have completed a conceptual design for a dual accelerator system. The first accelerator would be a low-energy $(<500 \mathrm{keV})$ high current ion source with variable acceleration gap. The adjustable acceleration gap has been designed to provide the necessary beam emittance over a wide range of acceleration potentials. To address the ion beam transport of the very high currents at the lowest energies $(<100 \mathrm{keV})$, a beam neutralization concept has been evaluated and validated. The second accelerator will be a commercially available Dynamitron source, with an ECR source mounted at the terminal to provide higher charge state beams and higher final beam energy. This is necessary to investigate helium-burning and neutron producing reactions. Particularly challenging components of the engineering design include provisions for supporting the weight of a compact ion source at the accelerator terminal and provision for vacuum pumping of this source inside the terminal enclosure vessel.

We have achieved a conceptual design for beamlines and target stations to provide for efficient use of time of the accelerators. Some cross section measurements require using both accelerators for measurements across a wide astrophysically significant energy range, and so the beamline provides a switching magnet to guide beam to a common target. On the other hand, some experiments will likely require very long run times to acquire statistically significant measurements, so that a second target station is desirable for simultaneous research and staging.

We have continued modeling and design of a supersonic gas jet target. The design is challenging since the ion beam final focus region is constrained by the focal properties of the final beamline elements and the size of high-efficiency detectors. Finite element analysis modeling of the supersonic gas jet flow has suggested a design for the jet nozzle and gas flow operating parameters (temperature and pressure) which should allow the jet size and density to meet the requirements of overlap with high-intensity beams. 


\section{Physical Biosciences Division}

LB08005

Understanding the Nano thermodynamics of Molecular Machines

Principle Investigators(s): Gavin E. Crooks

\section{Project Description}

The goal is to develop the necessary fundamental understanding of nanoscale, non-equilibrium thermodynamics so that we may understand the practical limits and operational principle of molecular machines, both biological and artificial. We wish to understand the thermodynamic efficiency of molecular machines, the tradeoff of efficiency with power output, how to determine the optimal thermodynamic processes, and how to engineer machines that can approach these optimal performances.

\section{Accomplishments}

We have completed our investigation of extending thermodynamic fluctuation theories to quantum dynamics. In two papers we have explored the technical machinery necessary to fully describe quantum effects.

We completed our current investigation into Bayesian estimates of free energies from non-equilibrium work measurements with a publication in J. Chem. Phys. There remain some intriguing technical points that need to be resolved, which may prove important. We have switched our attention to the more difficult problem of measuring free energy surfaces, potentially of means force, from similar experiments.

We have also initiated an investigation into the application of thermodynamic length, a concept from macroscopic finite time thermodynamics, to the limits of efficiency of microscopic systems. We hope to continue this research under a separate cover.

Finally, and unexpectedly, our investigations into non-equilibrium thermodynamics revealed new and profound insights into the nature of time, and the orientation of time's arrow, published in Phys. Rev. Lett. This work has in turn suggest a new avenue of investigation. It may be that thermodynamic efficiency at the molecular level is limited by the necessity of breaking time-reversal symmetry. 


\section{Light-boosted fermentation in the yeast Saccharomyces cerevisiae: Towards a science of cell architecture \\ PI: Jan Liphardt, Physical Biosciences Div. Co-PI: Manfred Auer, Life Sciences Div.}

Project Description. In the long term, transportation fuels may be synthesized on industrial scales with highly customized living cells. These cells will be constructed using synthetic biology approaches and will be fine-tuned to perform a small number of precisely defined reactions with great efficiency. Examples may include cells that convert $\mathrm{CO}_{2}$ and sunlight into biofuels.

To realize this prediction, it will be essential to discover and develop general principles that will ultimately give synthetic biologists control of cell shape and ultrastructure. Consider the photoreceptor cells in the human eye. There, the plasma membrane is folded back on itself numerous times. This "membrane shelving" increases the surface area of the cell membrane, and therefore, the capture cross-section for photons. Without this special shape of the cell, the human eye would be hundreds of times less sensitive to light, and we could not see at dawn or dusk. This is one of many examples where the shape or ultrastructure of a cell greatly enhances the function of an enzyme, such as the Rhodopsin protein in our photoreceptors.

Accomplishments. We have used the FY 2008 LDRD funding to create a strain of $S$. cerevisiae (Baker's yeast) that features extensive internal light-collecting structures. $S$. cerevisiae does not normally harvest light energy, but it is an efficient producer of ethanol and therefore a good model system to study conversion of glucose into biofuels or biofuel precursors. To our knowledge, this is the first time that a eukaryotic cell has been re-engineered to contain a multilayer membrane structure similar to the photoreceptors in our eyes or the chloroplasts in plants.

We constructed this synthetic light-harvesting structure by expressing a modified form of the Proteorhodopsin light-powered proton pump. Normally, the Proteorhodopsin enzyme pumps protons when illuminated, but it does not reconfigure the cell's ultrastructure. However, about 10 years ago a group working on an entirely different system, cytochrome $b(5)$, discovered that expression of GFPtagged cytochrome $b(5)$ lead to the formation of membrane stacks, so-called karmellae. We speculated that a GFP-tagged form of Proteorhodopsin might also exhibit this behavior.

To our surprise, this prediction was correct. As can be seen in the figure, proteorhodopsin constructs containing the native bacterial localization presequence and a nonmonomeric GFP tag form semicircular membrane aggregates which can be seen both in fluorescence and electron microscopy. By influencing membrane structure and creating membrane shelves, the proteorhodopsin-GFP construct transforms certain organelles of this heterologous organism, $S$. cerevisiae, in precisely the right way to maximize its proton-pumping efficiency.

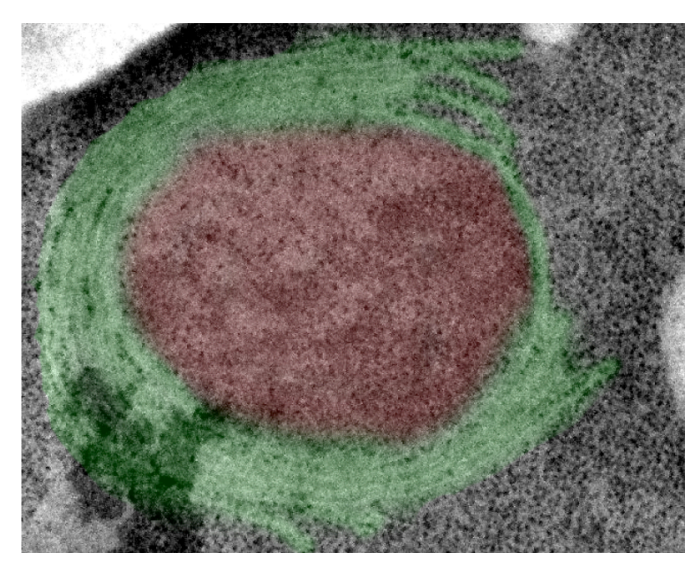

Discovery of the ability of GFP-tagged Proteorhodopsin to reconfigure internal membranes, creating light-collecting structures in yeast. Proteorhodopsin (with its native localization signal and fused to a nonmonomeric green-fluorescent protein) creates multi-lamellar semicircular membrane structures in yeast. False-color electron micrograph of a single yeast cell showing the syntheticallyencoded membrane compartment (3-5 layers thick, green) surrounding the nucleus (red).

Our goal in the next year (FY 2009) is investigate the possible metabolic implications of these membrane structures. Do they increase the cell's ability to harvest light? Our hypothesis is that the increased effective cross-section and light collection of the stacked membranes will increase the degree to which the cells can pump protons in response to illumination. Ultimately, we wish to couple these proton fluxes to the generation of cytoplasmic ATP, which is needed for enzymatic reactions such as carbon fixation and biofuel production. 
Cooperation of Biochemical and Mechanical Signals in Regulating Cell Fate Decisions During Tissue Morphogenesis

PI: David Schaffer, with Mina Bissell and Adam Arkin

Postdocs: Chinmay Pangarkar and Carole Berruyer-Pouyet

Project Description:

We have developed an interdisciplinary approach that unites multiple LBNL and UCB investigators from different disciplines to study how cells interact with each other and their microenvironment during tissue regeneration and repair. This effort has been bridging biomaterials development, mathematical modeling, and high impact biological systems in an integrated effort to advance our understanding of a very complex and important area of biology.

We focus on two central model systems that have many features in common, but a number of fundamental differences: (1) the branching and "invasion" of the mammary tree into the mammary fat pad, and (2) the migration and differentiation of neural stem cells (NSCs) to form new neuronal circuits in the adult brain. We investigate how the mechanical and biochemical signals that comprise the cellular microenvironment cooperate to regulate cell functions such as proliferation, migration, invasion, and differentiation. In particular, we have been investigating the roles played by the biochemical signaling system Eph/Ephrin in conjunction with matrix mechanical stiffness.

Accomplishments:

In mammary gland tissue, we find that expression of two Eph receptors is induced during puberty, when extensive ductal elongation and side-branching occurs. To assess the effect of Eph activation on cell proliferation, a process important for branching morphogenesis, we used Eph4 and ScP2 mouse mammary epithelial cell lines, which we find express the transcripts and the proteins of both receptors. Eph4 and Scp2 proliferation was inhibited by clustered ephrin ligands, a result that correlated with an inhibition of MAPK and FAK. To test the effect of Eph activation on the differentiation process, we have used Sca-1 positive cells isolated from the Comma-D $\beta$ cell line. When treated with EphrinB1, the Sca-1 positive cells upregulate keratin8, suggesting a role of ephrinB1 in the luminal differentiation of basal/stem cell-like cells. To assess a possible function for Eph receptors in alveolar differentiation we used primary mammary organoids in a 3-D Matrigel assay, we observed that Ephrin B1 prevents the formation of branched structures that normally develop upon stimulation with the growth factor TGF $\alpha$.

In NSCs, Ephrins B2 and B3, as well as Eph A2, drove cell proliferation. In contrast, EphrinB2 promotes neuronal differentiation to the strongest extent of any protein factor to date, an important result we are currently confirming in vivo. This signaling is dependent on a specific Eph receptor and requires the activity of ROCK kinase, a factor implicated in mechanotransduction. In parallel, we have used a new biomaterials system to demonstrate that the mechanical stiffness of the cellular environment strongly influences neuronal differentiation, and we are currently elucidating whether this effect is also dependent on ROCK and RhoA. Taken together, these results have made considerable progress in understanding how biochemical and mechanical systems play significant roles in determining the neuronal fate of NSCs.

Future objectives include final validation of these findings in vivo, confirmation of the relevant intracellular mechanisms, extension to 3D biomaterials systems, microarray analysis of mechanism, and additional mathematical modeling of both neural and breast cell function. In addition, we will continuously pursue NIH funding, building on the success of one grant to date. 


\section{Physics Division}

LB08028

Advanced Silicon Detectors for Future Short Pulse X-ray sources Principal Investigators: Marco Battaglia and Peter Denes

The LDRD program addresses the development of Si sensors of new concept for application at X-ray sources and high energy electron accelerators. R\&D programs towards next generation facilities, such as the BELLA project, require efficient beam monitoring with high particle fluence and fast detection of soft photons with energy and spatial information for fast nanoimaging at X-ray sources and beam diagnostics to novel particle acceleration techniques.

Emerging Si technologies offer in principle a path to solve the detection challenges from these applications. But advanced R\&D is required to connect new trends in semiconductor industry to the specifications of applications at accelerators. In the first year of this LDRD program the activity has been focused on the Silicon-On-Insulator (SOI) technology for the fabrication of monolithic pixel sensors tailored to fast imaging of soft radiation. The SOI process attracts significant interest in the semiconductor industry since it offers devices of higher speed and lower power consumption. In an SOI device a thin oxide layer insulates the device wafer from an handle wafer. For detector construction the handle wafer is a detectorgrade high resistivity $\mathrm{Si}$ wafer which acts as detection volume and the device wafer has the associated readout electronics. The insulation of the device layer from the detector volume offers important advantages compared to monolithic pixel sensors realised in standard CMOS bulk process. First the the implants for the readout electronics do not disturb the charge collection process and then the detector wafer can be reversely biased, increasing the sensitive volume and improving the charge collection properties.

The SOI technology in a deep-submicron CMOS process has recently become available through a collaboration with KEK, Tsukuba, Japan and semiconductor industries, to which our group has contributed with a pixel test structure which features $10 \times 10 \mu \mathrm{m}^{2}$ pixels with both analog and digital readout architecture. The first SOI monolithic pixel chip designed at LBNL and manufactured in the OKI $0.15 \mu \mathrm{m}$ FD SOI process has been tested on the ALSBTS 1.35 GeV electron beam. Signals have been observed on both the analog and digital pixels. This is the first pixel chip in SOI technology to observe particles from a beam. In addition, the radiation hardness of the SOI process has been assessed at BASEF at the 88-inch cyclotron. These first results are very encouraging for the further development of this technology for precise and fast radiation detectors as they demonstrate the feasibility of the SOI process for manufacturing pixel sensors and identify key areas where R\&D is needed. Following the succesful tests of this first SOI pixel chip, a second, more advanced prototype has been designed and manufactured. This will offer a larger sensitive area, fast readout capabilities and improved signal-to-noise performance.

The possibility to deplete several tens of microns in the SOI detector layer opens the perspective to fabricate a pixellated sensor with an extremely broad spectral response by thinning the detector wafer in the SOI device and implanting a thin entrance window by post-processing. The delta-doping process developed at NASA-JPL applies a mono-layer on the detector backplane and it has already been successfully applied to the LBNL thick CCDs. Thin-window SOI pixels with fast readout are ideal for measuring the $30 \mathrm{~nm}$ radiation from the proposed LBNL SASE FEL laser-driven accelerator. The applicability of the JPL backthinning and delta-doping processes has been studied in collaboration with the Advanced Detector and Nanoscience Technology group at JPL and a joint R\&D program is being deployed. 


\section{Effective Theories for Collider Physics}

Principal Investigator: Christian Bauer

\section{Project Description:}

In order to cleanly separate the physics we hope to discover at the large hadron collider, expected to start taking data next year, from the interactions we already understand, we need a detailed understanding of the expected standard model processes. In the past, these standard model processes were studied using traditional perturbative methods, and while significant progress has been made, the difficulty has always been to deal with the complicated structure of QCD. Over the past decade, significant progress has been made to understand QCD using the framework of effective field theories (EFTs). EFTs provide a systematic way of analyzing the underlying theory in particular limits of the theory. Recently, a new effective theory called soft-collinear effective theory (SCET) has been developed by myself and collaborators, and it has proven to be tremendously successful to understand QCD for highly energetic particles. The goal of this project is to apply SCET to understand the standard model backgrounds at the LHC, which most of the time contain highly energetic particles in the initial and final states. Using the tools of effective theories should allow for a clean understanding of the required processes, with the possibility of systematic improving the description as the need arises.

\section{Accomplishments:}

This year has seen the first release of GenEvA, a new framework for event generation. Event generators produce events similar to the actual events produced in actual experimental setups, but based on the underlying theoretical understanding of the standard model. This is crucial since this theoretical "data" can be processed through simulations of the detector effects, and can therefore be compared directly with the true experimental data. GenEvA uses the insight from SCET to combine perturbative results with parton shower algorithms. Most of the work was on the general setup of the framework, allowing to cleanly separate the theoretical calculations from the algorithmic details. Since the first release, we have spent most of our time deriving the theoretical expressions required to implement higher order perturbative calculations. We expect to implement these calculations into GenEvA in the coming months.

We have also finished work on so-called factorization proofs for processes involving hadronic initial states. Currently we are in the process of exploiting this new found understnding to make predictions for the most important theoretical backgrounds expected at the LHC. 


\section{Development of Nanowire Carpet Hybrid Pixel Detectors Principal Investigator(s): Maurice Garcia-Sciveres}

Project Description:

The purpose of this project is to produce and test prototypes that demonstrate the invention of a new fabrication process for silicon pixel radiation detectors. So called hybrid pixel detectors are currently fabricated by lithographically processing silicon sensor wafers, which is done by a variety of commercial providers. To produce a working detector, the lithographically produced sensors must be electrically connected to custom integrated circuits, which is currently done using fine pitch flip-chip bump bonding. By way of this invention, lithographically produced sensors are replaced with a silicon wafer that has been provided with a carpet of nano-wires covering the interface surface, these nano-wires making electrical contact with the readout chip. This work involves treating and then etching the surface of a special silicon wafer to produce electrical characteristics appropriate for radiation detection, followed by the investigation of methods to attached this new type of sensor to the readout chip. If successful, this invention would enable lower cost hybrid pixel detectors by eliminating both of the presently cost-driving steps of sensor lithographic processing and fine pitch bump bonding.

This work is supported by LDRD funding and benefits from access to Molecular Foundry (MF) equipment and advice through a MF instrument proposal The nano-fabrication techniques used are not new and have been learned from MF staff and other users. The novelty of this work lies in the application of nano-fabrication techniques to the production of working instruments that can currently only be produced only by less efficient and therefore more costly methods.

Accomplishments:

We have developed a recipe of wafer treatment steps and nano-wire formation and used it to reproducibly fabricate samples that have electrical properties adequate for use as radiation detectors. We have also illuminated samples with an alpha particle source and observed the expected signals. We have filed a provisional patent application and therefore do not give technical details here. We are presently investigating interconnection methods for permanent attachment of our sensor samples to existing readout chips from the ATLAS hybrid pixel detector that was built and installed at CERN. We expect to file a full patent once reliable interconnection has been developed. All electrical tests so far have been carried out with probetype contact (i.e. no mechanical attachment).

The fabrication and electrical study of samples has been carried out in the building 70 microsystems lab. The LDRD work is complemented by a Molecular Foundry proposal providing access to electron microscopy. The nano-wire formation methods have been investigated with significant consultation from MF staff and other users. Both growth techniques from gas phase at elevated temperature and room temperature wet etching methods have been explored. 
Development of Multi-Modular Assemblies with Reduced Material and Services for

Specifications at Future Particle Tracking

Principal Investigator(s): M. Gilchriese and C. Haber

\section{Project Description}

Future silicon tracking detectors, such as in a proposed Super Large Hadron Collider (SLHC), require the development of multi-modular mechanical support structures with integrated cooling and electronics on an unprecedented scale. The purpose of this project is to study a concept which can meet the requirements of such tracking systems. The concept is based upon thin, low mass, stiff composite laminated structures which embed both high performance cooling and electrical distribution of high bandwidth signals and efficient power distribution methods.

The project consists of two principal aspects. One of these is the design of integrated mechanical/cooling structures that could be applicable to all elements and regions of such a future silicon tracking system. These require both design studies (analytical calculations and finite-element calculations) and the fabrication of a number of prototypes to explore and test the design concepts. The second aspect is to assemble and test the electrical performance of prototype integrated structures using silicon strip modules.

\section{Accomplishments}

We completed conceptual designs for mechanical/cooling structures for all regions of a future SLHC-type silicon tracker based on a common design principle - the use of low-density, thermal conducting carbon foam coupled with readily available carbon fiber materials and metallic cooling tubes. A full-length $(1.07 \mathrm{~m})$ prototype mechanical/cooling structure with an attached electrical distribution cable was completed and provided for the electrical tests described in the next paragraph. Six small prototypes, applicable to the inner regions of a future SLHC-type silicon tracker, were fabricated and tested successfully. Fabrication of a full-size prototype for the outer (forward disk) region of a future tracker was initiated. The common design principle continues to be the leading candidate for a future SLHC-type tracker.

We have fabricated and tested an electrical stave structure using the mechanical/cooling prototype cores described in the preceding paragraph. Cooling fluid runs in through this structure and maintains it at a safe working temperature. We have fabricated hybrid circuits both in a Beryllia ceramic thick film technology and a thin kapton copper low mass laminate. We have demonstrated equal performance for both approaches which is also in specification for low noise behavior. We have glued these hybrids directly to the surface of silicon sensors and demonstrated electrical performance also in specification. We have demonstrated that an adhesive application to the sensor surface maintains the original high breakdown voltage. We have fabricated and tested a fine pitch high density bus cable structure. Modules have been mounted upon this structure and tested with good performance. We have continued the development of high bandwidth multi-channel data acquisition hardware and software to enable the readout of stave and modules. We have established sensor testing hardware and software and are preparing for a proposed sensor irradiation at the 88-inch Cyclotron in February 2009. We have developed and regularly use a set of precision assembly procedures by which modules are mounted on the stave structure and wirebonded. 


\section{LB08046}

Calibrating Baryon Oscillations for Future Experiments

PI(s) : Nikhil Padmanabhan, Martin White, David Schlegel

Project Description :

The baryon oscillation method is now an essential element of all programs to measure the expansion rate of the Universe and constrain the properties of the "dark energy" driving the recent accelerated expansion. The next generation of surveys, that will constrain the expansion to $1 \%$ at multiple redshifts, and the Joint Dark Energy Mission (JDEM), a DOE-NASA mission that will achieve $\sim 0.1 \%$ constraints, is already being planned. This project aims to provide the theoretical support for these experiments, by quantifying possible systematic errors and developing methods for optimally extracting the baryon oscillation signal from these data sets.

We aim to describe the effects of the (a) nonlinear clustering of the dark matter, (b) halos and galaxies, and (c) redshift space distortions on the acoustic feature. We do this both with large numbers of N-body simulations, varying simulation resolutions and cosmologies to ensure the robustness of our results. Combining these simulations with perturbative techniques, we aim to develop an analytical framework that captures the above effects at the fidelity required by the next generation of surveys. At the same time, we plan on developing new analysis techniques that could reduce these effects and quantify how such methods might drive the design of the next generation of surveys.

Accomplishments :

We have made significant progress on understanding how the nonlinear clustering (item a above) of the dark matter affects the baryon acoustic feature. We have run $\sim 1000 \mathrm{Gpc}^{3}$ of Nbody simulations (the next generation of experiments will survey $\sim 10 \mathrm{Gpc}^{3}$ of the Universe), across four very different cosmologies and for a variety of simulation parameters. This has allowed us to explicitly demonstrate that our current simulation techniques are accurate enough to correctly capture the physics on the scales of the baryon oscillations. Furthermore, we have used these simulations to demonstrate that the effect of nonlinear clustering on the baryon oscillations are accurately described by second-order (1-loop) perturbation theory. This allowed us to construct a analytic model for baryon oscillations in the nonlinear regime that is accurate to the $\sim 0.1 \%$ level necessary for future surveys. We are in the process of extending these methods to consider the effects that dark matter halos, galaxies and redshift space distortions have on the acoustic feature (items $\mathrm{b}$ and $\mathrm{c}$ above).

We have also made progress in quantifying the requirements of "reconstruction" methods (designed to undo some of the effects of nonlinear clustering) on the survey parameters. These results will have important implications when optimizing the design for the next generation of surveys. In addition, we have improved our analytic understanding of how these methods work and what their regimes of validity are. These efforts have also suggested improvements that can be made to such methods, an area for future research. 


\section{High-Yield CCD Process Development at the LBNL MicroSystems Lab \\ Natalie Roe, Physics Division \\ Steve Holland and Nick Palaio, Engineering Division}

Project Description: We proposed an R\&D project aimed at developing a more efficient and higher-yielding process for the fabrication of fully depleted charge-coupled devices (CCDs). A secondary goal was to enable the fabrication of thinner $(<200 \mathrm{um})$ devices. Fully-depleted CCDs are now the detector technology of choice for many planned and proposed large focal plane arrays for astronomical surveys dedicated to understanding the nature of dark energy, the unknown force responsible for the acceleration of the expansion of the universe. LBNL has partnered with a commercial CCD manufacturer for the fabrication of fully depleted CCDs, with the final processing steps on thinned wafers completed at LBNL in the MicroSystems Lab (MSL).

The limiting factor for the production of fully depleted CCDs is the processing time and yield losses incurred during the multiple processing steps in the MSL. Also, the current process flow is suitable for wafers of 200 um or more in thickness. We proposed to develop an alternate fabrication process based on the use of a refractory metal, Titanium Nitride (TiN), for the CCD metallization. More of the processing steps for TiN CCDs can be completed at a commercial foundry, reducing the processing time and handling in the MSL. We expect that yields can be doubled and the total MSL processing time can be cut in half with this new technology. In addition, this new process flow is suitable for wafers thinner than $200 \mathrm{um}$. In addition, we proposed to acquire a wafer saw in order to reduce persistent and uncontrolled yield losses that have occurred due to electro-static discharge (ESD) damage at our commercial dicing vendor.

Accomplishments: We ordered 48 wafers of TiN CCDs from our commercial partner, specifying a "Ti first" recipe for the TiN metallization in order to guarantee good contact resistance. Six wafers were delivered initially for test and development. We verified that the commercial processing was successful through wafer cold probe testing and also by packaging and testing CCDs from the control wafers. Three wafers were thinned and processed in the MSL according to the new procedure, which utilizes an upgraded furnace that was installed in the MSL this year using LBNL GPE funding. Initial results from the MSL indicated that the contact and passivation masks required minor modifications to avoid etching of some pads during batchmode processing. Scanning electron microscopy (SEM) of the affected bond pads was done to confirm the source of the problem. The mask changes were completed and submitted for incorporation into the commercial processing of the remaining 44 wafers.

We also purchased a reconditioned wafer-sawing machine that will allow us to dice the wafers with strict (ESD) precautions in place, under full control of LBNL staff. Installation and commissioning of the dicing saw in a dedicated room with an ESD-safe floor is in progress. 


\title{
Baryon Oscillations and Dark Energy: Prototyping Instruments
}

\author{
Principal Investigator: David Schlegel
}

Project Description

Future baryon acoustic oscillation (BAO) experiments for measuring dark energy witll require technologies to control more than 1000 fibers on the focal plane of a telescope. A simple, cost-effective approach is to individually actuate each fiber in the focal plane. This removes the complication and expense of drilling plug plates (like for the Sloan Digital Sky Survey) or building robots to move fibers meter distances with the required $30 \mu \mathrm{m}$ tolerance.

\section{Accomplishments}

The initial prototype of the LBNL fiber actuator has been completed, with the exception of the fiber mounting mechanics. Construction of the prototype required some custom machining and re-design from the original conceptual drawings. The rotation motor uses a pinion and static inside gear; the radial translation motor drives a rack with a triple pinion. The backlash of the MicroMo motors have been thoroughly characterized, and a control system devised that minimizes power dissipation by powering down the motors while preserving position information.

The LBNL fiber actuator was presented at the June 2008 SPIE meeting, including a demonstration of the working prototype. The design was published in the SPIE meeting proceedings.

A collaboration with the Instituto de Astrofisica de Andalucia (IAA) in Granada, Spain, is now developing a variant of the LBNL fiber actuator for use on the Spanish 10.4-m telescope. That design would make use of 1000 fibers in a larger $99 \mathrm{~cm}$-diameter focal plane, with a center-to-center spacing of $2.92 \mathrm{~cm}$ instead of $1.92 \mathrm{~cm}$. The additional design work was funded by IAA in early 2008. Additional funding for a 19 actuator focal plane was secured from the Spanish government in July 2008. A preliminary design review of the IAA actuator was performed by LBNL in September 2008. 


\section{Decoding Dark Energy with Weak Gravitational Lensing \\ Principal Investigators: George Smoot, Alexie Leauthaud}

\section{Project Description}

Understanding the nature of dark matter and dark energy represents two outstanding challenges for the new century. Whereas compelling evidence suggests that dark matter is non-relativistic and collisionless thus guiding our quest for the appropriate particle the general properties of dark energy remain far more elusive. There are four main potential probes of the dark energy equation of state: SNe Ia distance measurements, Baryon Acoustic Oscillation (BAO), features in the spatial distribution of galaxies, the abundance of galaxy clusters, and weak gravitational lensing of large-scale structure. We propose a comprehensive study of weak lensing as one of the most powerful means to study dark energy. We will carry out this study in two steps: first by analyzing the ability of weak lensing to directly detect massive clusters and second, by comparing these results to various other weak lensing statistics that are also sensitive to the dark energy equation of state.

\section{Accomplishments}

In 2008 we have prepared a study of the weak lensing signal around groups and clusters in the COSMOS field (see Figure 1). Groups have been stacked according to their Xray Luminosity $\left(L_{X}\right)$ which allows for a better understanding of the relationship between the hot intra-cluster gas and dark matter halos. This study is an important step forward towards better measurements of the abundance of galaxy clusters as a function of halo mass. These results form the basis of publication that will soon be submitted to the Astrophysical Journal. Building on this carefully calibrated scaling relation, we have started a study of the abundance of clusters and groups within the COSMOS field to check if our results are in agreement with the current cosmological model.

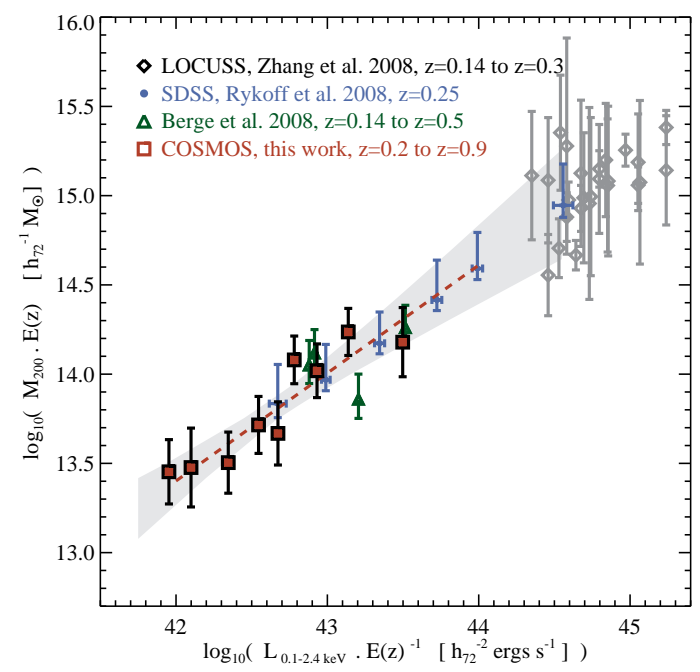

Figure 1: The relationship between X-ray Luminosity and Halo mass as measured by weak gravitational lensing in the COSMOS survey. 


\title{
Cross-Divisional
}

LB08047

\section{Multiscale Imaging of termite hind gut microbial biofilms}

\author{
PI: Dr. Manfred Auer (Life Sciences Division); co-PI: Dr. Jan Liphardt (Physical Biosciences \\ Division) in collaboration with Dr. Phil Hugenholtz (Joint Genome Institute)
}

\section{Project description:}

The termite hindgut microbial community is one of nature's most efficient lignocellulose biodegrading "factory". Metagenomic analysis revealed that it is composed of about two hundred microbial species displaying a high degree of redundancy of lignocellulolytic activities. By studying the spatial and temporary distribution of lignocellulose degrading microbes we will obtain fundamental insight into the depolymerization process which will guide synthetic biology efforts to mimic nature in an industrial process to efficiently breakdown lignocellulose for biofuel production. Multimodal and multiscale imaging techniques, such as correlative 16S rRNA Fluorescence In-Situ Hybridization (FISH), Raman imaging and electron microscopy (EM), will be necessary to identify a spatial and temporal map of hind gut community members and their macromolecular machines responsible for efficient cellulose degradation.

Our first specific goal is to establish by correlative fluorescence in-situ hybridization (FISH) and electron microscopy (EM) whether microbial species in the termite hindgut, including rare and unexpected phylotypes, reflect indeed the necessary metabolic partitioning of spatially and temporary required steps involved in efficient lignocellulose degradation. From this analysis we will determine whether some (or most) microbes in the termite hindgut are dispensable while maintaining efficient cellulose degradation. Second, we will assess the modularity of the termite microbial community. Specifically, we will ask whether the microbes are organized into processing units with chemical micro-environments, and whether some of those processing units are simple enough to be replicated in a commercial setting. This knowledge will be of great interest to synthetic biologist in JBEI and other BioEnergy Research Centers. Third, we will ask whether lignocellulose degradation in the hindgut involves vesicle-encapsulated biocatalysts and other macromolecular machines. If such lignocellulose degradation-relevant extracellular structures will be detected and characterized in the termite hindgut, their activity will be of high commercial significance.

\section{Accomplishments:}

We have successfully preserved the termite hindgut microbial community in epoxy and LR White resin for EM imaging, Raman Imaging and FISH labeling. We have surveyed at high resolution large areas ( 100 by 100 microns), with well over a 1,000 bacteria of various shapes and size. We have found that certain cell morphologies are predominantly associated with plant cell wall debris, whereas others are more closely associated with the termite gut epithelial tissue or fill the space between the plant biomass. These filamentous bacteria tend to orient themselves with their cell pole towards the biomass and show close packing. Furthermore we have been able to visualize an astounding array of extracellular features, including macromolecular machines very likely involved in lignocellulose degradation. In addition, we found discovered vesicles at the site of bacterial adhesion to the cell wall material, suggesting that certain cellulolytic activities may be delivered via membrane vesicles. Besides the ultrastructural findings, we have begun to develop FISH protocols for resin-embedded samples, which -once robust- will allow the identification and precise spatial mapping of each microbial community member. 
Do CRISPR Regions of Bacteria and Archaea Constitute an RNAi Based Immune System? Principal Investigator(s): Stephen R. Holbrook (PBD), Jennifer Doudna (PBD), Jill Banfield (ESD), Steven M. Yannone (LSD)

Project Description:

Mounting evidence suggests that bacteria and archaea have evolved an 'RNA-based adaptive immune system' that might be functionally analogous to the RNAi system in eukaryotes; however the participants and molecular mechanisms that are important at each step of this immune system have not been established. The goal of this project is to understand the molecular mechanism by which CRISPRs (Clusters of Regularly Interspaced Short Palindromic Repeats) assimilate new viral sequences and how newly acquired CRISPR spacer sequences are implemented as homing oligos in an immune response that confers phage resistance. This work will reveal potential similarities with eukaryotic RNAi pathways and will provide mechanistic insights that will be important to engineering phage resistant strains of bacteria that are currently being grown to high densities for applications in biotechnology, bioremediation, and bioenergy.

Approach/Methods:

We will exploit four different model systems to determine how CRISPRs and their associated proteins provide immunity to parasitic genetic elements. Three of these models are three culture based systems including Sulfolobus solfataricus, Streptococcus thermophillus, and Pseudomonas aeruginosa. These phylogenetically diverse culture-based systems have been complemented with a culture-independent approach. A natural microbial community, dominated by Leptospirillum will be analyzed using a metagenomic approach to define the role of CRISPR-based immunity in the natural environment.

Accomplishments:

Major accomplishments form LDRD support for FY2008 include the high-resolution crystal structure and biochemical characterization of a universally conserved Cas protein (Cas1). The Cas1 structure reveals a novel three-dimensional fold and the biochemical characterization of this protein suggests that Cas1 is a metal dependent, double-strand DNA-specific endonuclease. The structure and function of Cas1 are consistent with a role for this protein in the recognition and processing of foreign DNA. This work represents a major advance in gaining a mechanistic understanding of how this immune system works.

Major accomplishments from the community analysis reveal a dynamic interaction between CRISPRs and the phage populations targeted by this immunity system. This work demonstrates that CRISPRs are a rapidly evolving component of the microbial immune system and that phage respond to the selective pressure imposed by this immune system by recombining their genetic material to escape detection. 
Fabrication of photovoltaic devices using genetically engineered biomaterials Principal Investigator(s): Seung-Wuk Lee

\section{Project Description}

The purpose of this project is to fabricate virus-based photovoltaic devices to harness novel and clean solar energy. The monodisperse photovoltaic semiconductor nanowires will be synthesized through a mineralization process on genetically engineered viruses. Photovoltaic devices will be fabricated using semiconductor covered-virus nanowires through a conventional organic-inorganic hetero junction photovoltaic solar cell fabrication process. The approach will exploit combinatorial phage display to identify specific binding peptide information against photovoltaic semiconductor materials $\left(\mathrm{CdTe}, \mathrm{ZnO}, \mathrm{TiO}_{2}\right.$, etc), which will direct mineralization of such materials in mild aqueous conditions. Using this tactic, we will engineer monodisperse viral particles to template photovoltaic semiconducting materials. These viruses will then be vertically self-assembled on transparent electrodes to fabricate solar cell devices.

We will develop virus-based organic-inorganic hetero junction photovoltaic devices, by exploiting viruses (M13 bacteriophage) engineered to have specific affinity between major coat protein (pVIII) and photovoltaic semiconducting nanomaterials. The biological approach to build the proposed photovoltaic solar cell materials and devices has many promising aspects: 1) we can isolate previously unexplored novel peptide information against photovoltaic semiconducting material. Using the identified peptide information, we can design novel organisms, microbes, and biomaterials, with which we can open new fields of synthetic biology and couple many conventional micro-fabrication approaches (such as, soft lithography, DPN lithography, microprinting, and microfluidics) to build novel devices through bottom up processes. 2) Using recombinant DNA techniques, we can tune the length of the virus and subsequent nanowire scales which is suitable for the optimized device fabrication. In addition, cloning nature of the biological materials can simplify preparation of the sample through a self-replicating amplification processes. 3) Due to the diamagnetic property of the virus, we can align them vertically between the electrodes using external magnetic filed in the hetero junction devices, which is expected to enhance the efficiency of the photovoltaic devices by improving electron mobility. 4) Obtaining knowledge between biomolecules and electronic materials is critical for the design of novel nanoscale bio-electronic devices. 5) This multifaceted research project will provide graduate and undergraduate students with many opportunities to explore, integrate and educate the next generation of scientists in a variety of diverse research disciplines.

\section{Accomplishments}

We successfully constructed major coat engineered phage which are fully covered by the various functional peptides ( 4 glutamic acids, 4 histidine, and His-Pro-Gln(HPQ) tripeptide) and library sequences. The engineered phage with 4 glutamic acids can mineralized ZnS nanocrystals by incubating zinc and sulfide precursor ions solution. Additionally, the engineered phage with HPQ peptide could bind streptavidin-conjugated nanoparticles, which make it possible to modify the phage to many different types of nanowires without mineralization processes. Currently, we are characterizing the optical properties of the phage based $\mathrm{ZnS}$ nanowires and their effect of magnetic field to make them oriented in vertical direction for the fabrication of the composite materials on the ITO electrodes. We will investigate the miscibility of the phage-based nanowires with photovoltaic polymers (MEH-PPV). 


\section{Coupled Process Models, Separations, Monitoring for Advanced Nuclear Fuel Cycles}

Principal Investigator(s): Andrew Canning, Phil Colella, Per Peterson, Lee Schroeder (PI), Jim Siegrist, David Shuh, Jasmina Vujic, Niels Jensen (UC Davis)

\section{Project Description:}

Advanced nuclear fuel cycles require improved modeling and performance monitoring tools to optimize the fuel cycle, reactor design and licensing, and waste storage and disposal. This project will develop next-generation simulation models for reactor elements and licensing, model fuel cycle actinide behavior and develop instrumentation for the non-invasive study of waste forms and related radioactive materials critical to the nation's nonproliferation/safeguard activities.

Collaborations between LBNL computational scientists and UC Nuclear Engineers will focus on a coupled thermal hydraulics and neutronics model leveraging computational fluid dynamics expertise for design and licensing activities of reactors and fuel cycle facilities. Computational and chemical scientists carry out joint efforts to develop and study a complementary model of actinides in advanced fuel cycle separation systems. Novel instrumentation development of accurate non-invasive on-line monitoring systems, operating in the high radiation environments fuel rods, will be carried out.

\section{Accomplishments:}

Implemented a 3-D adaptive mesh refinement code to solve the heat equation in multiple phases, using efficient multi-grid solvers. The code was verified for simple geometries and contrasts of up to $10^{4}$ in the diffusion coefficients in the two phases. Also verified the ease and accuracy of geometry generation for wire-wrapped fuel elements.

Reviewed literature and USNRC reports related to the application of simulation results in licensing, to provide the basis to develop a generalized framework for using advanced simulation results. The review included how the role of simulation will change as licensing requirements shift from deterministic, conservative methods toward performance-based standards. Also effort was focused on current practices to benchmark that computed results accurately predict responses of complex systems such as reactors, fuel cycle facilities and geologic repositories.

The coordination chemistry of uranium in both low valence, and higher oxidation states, was explored with tri-dentate nitrogen-donor ligands. A new rich vein of coordination chemistry has been revealed with characterization of a number of new monomeric, dimeric complexes and also a mixed valence $U$ trimer. Theoretical work focused on new ligand complexes to understand actinide f-electron bonding in $\mathrm{U}$ and $\mathrm{Pu}$. First principal studies of $\mathrm{Pu}$ and $\mathrm{Ce}$ in Maltol and $\mathrm{Br}-$ Maltol ligand complexes were performed. Various first principle calculations of stability and suitability, in the trivalent separation of actinides and lanathanides for advanced fuel cycles, were performed.

Studied the isotopic assaying of spent nuclear fuel, an important capability for shipper/receiver verification and the independent measurement of the Pu input to the processing stream for materials accountability. Active interrogation methods were evaluated with the focus on nuclear resonance fluorescence (NRF) for measuring the isotopic content of spent fuel rods. First modeling results indicate NRF signals well above background even for isotopic fractions $<1 \%$ and thus the potential of NRF-based techniques. 


\section{Publications List}

AFRD-Fawley et al LB07015 FEL Concepts for Multiple Independent X-ray Beamlines

A. Belkacem, J. M. Byrd, J. Corlett, W. Fawley, J. Kirz, S. Lidia, W. McCurdy, H. Padmore, G. Penn, I. Pogorelov, J. Qiang, D. Robin, F. Sannibale, R. Schoenlein, J. Staples, C. Steier, M. Venturini, W. Wan, R. Wilcox, A. Zholents; "Design Studies for a HighRepetition-Rate FEL Facility at LBNL," Synchrotron Radiation News, Vol. 20, No.6, 2007.

W. Fawley; "Production of Ultrashort FEL XUV Pulses via a Reverse Undulator Taper," Nucl. Instr. Meth. A593, 111 (2008); LBNL-63558.

W. Fawley; "A Simple Estimate of Acceptable Emittances and Energy Spread for a High Gain, Soft X-ray FEL using M. Xie Estimates for Saturated Power," CBP Tech Note 388.

AFRD-Ji LB07017 Ultra-compact Field Desorption Neutron Source for Cancer Research

Y. Wu, L. Ji, and Q. Ji; "Ultra-compact field desorption neutron source," 2008 Symposium on Radiation Measurements and Application (SORMA West 2008)," June 2-5, 2008, Berkeley CA; presented as poster and manuscript submitted.

AFRD-Schenkel LB08017 Quantum Information Science with Integrated Color Centers in Diamond

C. D. Weis, A. Schuh, A. Batra, A. Persaud, I. W. Rangelow, J. Bokor, C. C. Lo, S. Cabrini, E. Sideras-Haddad, G. D. Fuchs, R. Hanson, D. D. Awschalom, and T. Schenkel; "Single-atom doping for quantum device development in diamond and silicon," J. Vac. Sci. Techn. B, in press, http://arxiv.org/abs/0806.2167.

G. D. Fuchs, V. V. Dobrovitski, R. Hanson, A. Batra, C. D. Weis, T. Schenkel, and D. D. Awschalom; "Excited state spectroscopy using single spin manipulation in diamond," Phys. Rev. Lett. 101, 117601 (2008), http://scitation.aip.org/getabs/servlet/GetabsServlet?prog=normal\&id=PRLTAO0001010 $\underline{00011117601000001 \& \text { idtype }=\text { cvips\&gifs }=\text { yes }}$

AFRD-Toth et al LB08022 Experimental Demonstration of a Laser-Plasma-Accelerator Driven Free-Electron Laser

Refereed Publications

C. B. Schroeder, E. Esarey, E. Cormier-Michel, W. P. Leemans; "High-harmonic generation in cavitated plasmas," Phys. Plasmas 15, 056704 (2008); LBNL-140E.

J. van Tilborg, C. Toth, N. H. Matlis, G. R. Plateau, W. P. Leemans; "Single-shot measurement of the spectral envelope of broad-bandwidth terahertz pulses from femtosecond electron bunches," Opt. Lett. 33, 1186 (2008).

Conference Proceedings

C. B. Schroeder, W. M. Fawley, F. Gruner, M. Bakeman, K. Nakamura, K. E. Robinson, Cs. Toth, E. Esarey, and W. P. Leemans; "Free-Electron Laser Driven by the LBNL LaserPlasma Accelerator," in Advanced Accelerator Concepts 2008 (AIP, in press). 
M. S. Bakeman, C. B. Schroeder, K. E. Robinson, Cs. Toth, K. Nakamura, W. M. Fawley, and W. P. Leemans; "Magnetic Characterization and Design of an Undulator-Based Electron Beam Diagnostic," in Advanced Accelerator Concepts 2008 (AIP, in press).

D. Panasenko, A. J. Shu, C. B. Schroeder, A. J. Gonsalves, K. Nakamura, N.H. Matlis, E. Cormier-Michel, G. Plateau, C. Lin, C. Toth, C. G. R. Geddes, E. Esarey, and W. P. Leemans; "Staging Laser Plasma Accelerators for Increased Beam Energy," in Advanced Accelerator Concepts 2008 (AIP, in press).

Cs. Toth, O. Albert, L. Canova, A. J. Gonsalves, D. Panasenko, N. H. Matlis, G. Plateau, and W. P. Leemans; "Contrast Enhancement of the LOASIS CPA Laser and Effects on Electron Beam Performance of LWFA," in Advanced Accelerator Concepts 2008 (AIP, in press).

\section{Invited Talks}

W. P. Leemans; "High-Energy Particle Accelerators that Can Fit on a (Large) Tabletop by Using Lasers," Colloquium, U. Chicago, Jan. 10, 2008.

W. P. Leemans; "High-Energy Particle Accelerators that Can Fit on a (Large) Tabletop by Using Lasers," Argonne National Laboratory Physics Division Colloquium, ANL, Jan. $11,2008$.

W. P. Leemans; Invited talk, Health Physics Society Annual Meeting, Sacramento, CA, March 28, 2008.

W. P. Leemans; "High energy particle accelerators that can fit on a (large) tabletop by using lasers," APS Spring Meeting, St Louis, Missouri, April 11-15, 2008.

C. Toth; "1 GeV electron beams from a laser-driven channel-guided accelerator," 2008 Quantum Electronics and Laser Science Conference, San Jose, CA, May 9, 2008.

W. P. Leemans; "High Quality GeV Electron Beams from Plasma-Laser Accelerators," 11th European Particle Accelerator Conference, EPAC'08, Genoa, Italy, June 22-27, 2008.

C. B. Schroeder; "Laser-plasma-accelerator-based radiation sources," 20th International Conference on the Application of Accelerators in Research and Industry, Fort Worth, TX, August 2008.

C. G. R. Geddes; "Laser-driven plasma accelerator experiments: injection and guiding," AAC'08 - Advanced Accelerator Concepts Workshop 2008, Santa Cruz, CA, July 2008.

D. Panasenko; "Control, Stability and Staging in Laser Wakefield Accelerators," LINAC2008 - XXIV Linear Accelerator Conference, Victoria, BC, Canada, September 2008.

E. Esarey; "Free-electron laser driven by the LBNL laser-plasma accelerator," Shanghai Synchrotron Radiation Facility, Shanghai, China, Oct 31, 2008.

AFRD-Vay et al LB08023 Lorentz Compaction of Scales for Ultra-efficient Simulation of Advanced Accelerators (and other systems)

J.-L. Vay; "Simulation of beams or plasmas crossing at relativistic velocity," Phys. Plasmas 15, 056701 (2008).

W. M. Fawley, J.-L. Vay; "Use of the Lorentz-boosted frame transformation to simulate freeelectron laser amplifier physics," Proceedings of the $13^{\text {th }}$ Advanced Accelerator Concepts Workshop, July 27-August 2 2008, Santa Cruz CA. 
AFRD-Wan et al LB07031 High Brightness Photocathodes as Electron Sources for FELs

E. Pedersoli, et. al; "Surface and Bulk Contribution to Cu(111) Quantum Efficiency," Appl. Phys. Lett. 93, 183505 (2008).

H. Padmore; "Improving Photocathodes with Nanostructuring," LSBL-913 (2008).

H. Padmore; "Reflectivity of an Aluminum Grating with Very Small Slots in the Ultraviolet Region," LSBL-912 (2008).

AFRD-Zholents et al LB07034 Emittance Manipulation and Beam Conditioning for FELs

A.A. Zholents, E. Kur, G. Penn, Ji Qiang, M. Venturini, R. P. Wells; "Linac Design for an Array of Soft X-ray Free Electron Lasers," in Proceedings of Linac 2008, Sep. 29 - Oct. 3, Victoria, British Columbia, Canada.

J. Qiang, R. Ryne, M. Venturini, A. A. Zholents; "Billion particle linac simulations for future light sources," in Proceedings of Linac 2008, Sep. 29 - Oct. 3, Victoria, British Columbia, Canada.

W. Fawley; "CSR-induced Emittance Growth in the LBNL FLS --- A Sample Estimate based upon a Single Chicane Bunch Compressor," CBP Tech Note-385.

M. Venturini; "Shot-Noise Seeded Microbunching Instability: Second-Order Correction to the Gain Function," accepted for publication in Nucl. Instrum. Methods Phys. Res. A; LBNL-676E.

M. Venturini; "Models of Longitudinal Space-Charge Impedance for the Study of the Microbunching Instability," Phys. Rev. ST Accel. Beams 11, 034401 (March 2008); LBNL-63108.

M. Venturini and A. Zholents; "Modeling microbunching from shot noise using Vlasov solvers," Nucl. Instrum. Methods Phys. Res. A 593, 53(2008); LBNL-567E.

M. Migliorati, A. Schiavi, G. Dattoli, and M. Venturini; "A 4D Vlasov Solver for Microbunching Instability in the Injection System of X-ray FELs," Proceedings of the European Particle Accelerator Conference, Geneva, (2008); LBNL-702E.

ALS-Guo LB07016 Building In-Situ Electronic Structure Study Capability with Photon-in/ Photon-out Soft X-ray Spectroscopy

\section{Publications}

Jun Zhong, Jauwern Chiou, Chungli Dong, Li Song, Chang Liu, Sishen Xie, Huiming Cheng, Way-Faung Pong, Chinglin Chang, Yangyuan Chen, Ziyu Wu, Jinghua Guo; "Probing quantum confinement of single-walled carbon nanotubes by resonant soft-x-ray emission spectroscopy," Appl. Phys. Lett. 93, 023107 (2008).

J. Guo; "Electronic Properties of Hydrogen Storage Materials with Photon-in/Photon-out Soft-X-Ray Spectroscopy,” J. Phys. Chem. Solids 69, 2223 (2008).

\section{Invited Talks}

J. Guo; "Photon-in/photon-out soft-x-ray spectroscopy: the coming in-situ tool for surface and interface science," Surface Science Seminar Series, Molecular Foundry, LBNL, November 15, 2007.

J. Guo; "Photon-in/photon-out soft-x-ray spectroscopy: the coming in-situ tool for surface and interface science," Surface Science Seminar Series, Molecular Foundry, LBNL, November 15, 2007. 
J. Guo; "Molecular Adsorption on SWNTs Probed Photon-in/Photon-out Soft-X-Ray Spectroscopy," RIXS08, Uppsala, Sweden (June 13-14, 2008).

P.-A. Glans and J. Guo; "Hydrogen Adsorption on the Surface of HOPG and SWNTs Studied by Soft-X-Ray Spectroscopy," SPIE - The International Society for Optical Engineering, San Diego, California, Solar Energy + Technology in SPIE 2008: Optics and Photonics, (August 10-14, 2008).

ALS-Hexemer et al LB08009 Soft X-ray Scattering as a New Probe of Polymer Systems

C. Wang, S.E. Harton, T. Araki, H. Ade; "Direct Analysis of the Effects of Isotopic Labeling on Polystyrene/Poly(Methyl Methacrylate) Interfaces Using Resonant Soft X-Ray Reflectivity," submitted to Macromolecules.

S. Swaraj, C. Wang, T. Araki, G. Mitchell, L. Liu, S. Gaynor, B. Deshmukh, H. Yan, C. R.McNeill, H. Ade; "Extension off the Utility of Resonant Soft X-Ray Scattering and Reflectivity or Nanoscale Characterization of Polymers," submitted to European Journal of Physics.

H. Ade, C.Wang, A. Hexemer, A. Garcia, T.-Q. Nguyen, G. Bazan, K. Sohn, E.J. Kramer, "Characterization of a Multicomponent Polymer Multilayer," to be submitted to Journal of Polymer Science.

C. Wang, A. Hexemer, A. Garcia, T.-Q. Nguyen, G. Bazan, K. Sohn, E.J. Kramer, H. Ade, "Using Resonant Soft X-ray Reflectivity to study the Interfaces of Conjugated Polymer Optoelectronic Devices," in preparation to be submitted to Advanced Materials.

ALS-Rotenberg LB08016 Carbon-Based Nanostructures as a Prototype for New Electronics

A. Bostwick, T. Warwick, E. Rotenberg, Y. Zhang, M. Crommie, N. Alem, A. Zettl; "AngleResolved Photoemission Investigation of electronic properties of exfoliated graphene microcrystals," in preparation.

ALS-Yashchuk LB07033 Ultra-high Resolution Optics for Soft X-ray Inelastic Scattering

H. Padmore, D. Voronov, R. Cambie, V. Yashchuk, E. Gullikson; "Ultra-high density diffraction grating," IB-2523P/LBNL043P; Provisional Patent Application Ser. No: 61/084,551; date filed: July 29, 2008.

D. Voronov, R. Cambie, E. Gullikson, V. Yashchuk, H. Padmore, Y. Pershin, A. Ponomarenko, V. Kondratenko; "Fabrication and characterization of a new high density Sc/Si, multilayer sliced grating," Proc. of SPIE 7077, 7077-7 (2008).

D. Voronov, R. Cambie, E. Gullikson, V. Yashchuk, H. Padmore, Y. Pershin, A. Ponomarenko, V. Kondratenko, "Fabrication and characterization of a new high density $\mathrm{Sc} / \mathrm{Si}$, multilayer sliced grating," abstract to Optics and Photonics 2008: Optical Engineering and Applications Symposium. Conference 7077-7: Advances in X-ray/EUV Optics and Components III (San Diego, August 10-14, 2008).

A. Aquila, J. Meyer-Ilse, R. Cambie, E. Gullikson, H. Padmore, D. Voronov, V. Yashchuk; "Resolution Measurements with a Sc/Si Sliced Grating," Light Source Beam Line Note: LSBL-923 (October, 2008).

D. Voronov, R. Cambie, E. Gullikson, H. Padmore, V. Yashchuk, M. Ahn, C.-H. Chang, R. Heilmann, M. Schattenburg, "Scanning Probe Microscope measurements with an MIT 200 nm grating,” Light Source Beam Line Note: LSBL-910 (September, 2008). 
D. Voronov, R. Cambie, R. Feshchenko, E. Gullikson, H. Padmore, F. Salmassy, A. Vinogradov, V. Yashchuk; "High resolution sliced multilayer grating for soft X-rays," abstract in the Digest of the 9th International Conference on the Physics of X-Ray Multilayer Structures (Montana, February 3-7, 2008).

CH-Arnold LB07003 Synthetic and Electrochemical Approaches to Metal-Metal Bonds in Actinides

S. G. Minasian, J.L. Krinsky, V.A. Williams, J. Arnold; “A Heterobimetallic Complex with an Unsupported Uranium(III)-Aluminum(I) Bond: (CpSiMe $)_{3} \mathrm{U}_{-} \mathrm{AlCp}$ *, J. Am. Chem. Soc. 130, 6456-6460 (2008).

CH-Bergman LB07006 Conversion of Glycerol and Aromatic Compounds from Biomass to Major 3-and 6-Carbon Industrial Organic Compounds

\section{Patent Disclosure}

R. Bergman, J. Ellman, E. Arceo Rebollo: "Conversion of Glycerol from Biodiesel

Production to Allyl Alcohol," provisional patent disclosure, application no. 60/866,661, filed January 26, 2007; published as WO 2008/092115 in the International Bureau of the World Intellectual Property Organization (WIPO) of the Patent Cooperation Treaty on July 31, 2008.

E. Arceo Rebollo, J. Ellman, R. Bergman; "Preparation of Alkenes and Other unsaturated Carbon Compounds from Polyhydroxy Compounds," record of invention form submitted to LBNL dated 03/04/2008.

\section{Publication}

E. Arceo Rebollo, R. Bergman, J. Ellman; "An efficient method for the conversion of glycerol to allyl alcohol and evidence for the mechanism of glycerol deoxygenation," in preparation for submission to Science.

CH-Bluhm, Wilson LB07009 Chemical Reactions at Liquid/Vapor Interfaces Probed by Photoemission Spectroscopy

D.E. Starr, E.K. Wong. D.R. Worsnop, K.R. Wilson, H. Bluhm; “A combined droplet train and ambient pressure photoemission spectroscometer for the investigation of liquid/vapor interfaces," Phys. Chem. Chem. Phys. 10, 2093 (2008).

CH-Toste LB08021 Metal Nitrosyl Complexes and Catalytic C-H Bond Functionalization

J. Schomaker, W. C. Boyd, I. Stewart, F. D. Toste, R. Bergman; "Cobalt Dinitrosoalkane Complexes in the C-H Functionalization of Olefins," J. Am. Chem. Soc. 130, 3777-3779 (2008).

CS-Agarwal, Guok LB07007 On-demand Overlays for Scientific Applications

D. Gunter, C. Guok, K. Jackson of LBL and M. Swany and E. Kissel at U Del; publication to be prepared. 
CS-Bell LB07005 Structured, Adaptive Mesh Refinement Method for Multiphase Reactive Transport in Groundwater

A. Almgren, J. Bell, G. Pau, and M. Lijewski; "Adaptive Mesh Refinement for Incompressible Flow in Porous Media," submitted to Journal of Computational Physics.

D. E.A. van Odyck, J. Bell, F. Monmont, N. Nikiforakis; "The Mathematical Structure of Multiphase Thermal Models of Flow in Porous Media," accepted for publication in Proceedings of the Royal Society A.

CS-Bell, Pau LB08003 Low Order Models and Numerical Methods for Computational Chemistry

G. S. H. Pau; "Reduced Basis Method for Nanodevices Simulation," Phys. Rev. B 78, 155425 (2008).

CS-Carter et al LB08035 Enhancing the Effectiveness of Manycore Chip Technologies for High-End Computing

K. Datta, M. Murphy, V. Volkov, S. Williams, J. Carter, L. Oliker, D. Patterson, J. Shalf, K. Yelick; "Stencil Computation Optimization and Auto-tuning on State-of-the-Art Multicore Architectures," Proc. of High performance computing, networking, and storage conference, November 2008.

S. Williams, J. Carter, L. Oliker, J. Shalf, K. Yelick; "Lattice Boltzmann Simulation Optimization on Leading Multicore Platforms," Journal of Parallel and Distributed Computing (to appear).

CS-Markowitz LB06015 Integrated Microbial Community Genomes Data Management System

Publications

Metagenome Data Management and Analysis Tools

V.M. Markowitz, N. Ivanova, E. Szeto, K. Palaniappan, K. Chu, D. Dalevi, I.M. Chen, Y. Grechkin, I. Dubchak, I. Anderson, A. Lykidis, K. Mavromatis, P. Hugenholtz, N.C. Kyrpides; "IMG/M: a data management and analysis system for metagenomes," Nucleic Acids Research 36, D534 - D538 (2008).

D. Dalevi, N.N. Ivanova, K. Mavromatis, S. Hooper, E. Szeto, P. Hugenholtz, N.C. Kyrpides, and V.M. Markowitz; "Annotation of metagenome short reads using proxygenes," accepted for publication in Bioinformatics 24 (18).

I. Sharon, A. Pati, V.M. Markowitz, R.Y. Pinter; "A statistical framework for the functional analysis of metagenomes," submitted to RECOMB 2009, International Conference on Research in Computational Molecular Biology.

Metagenome Studies using Analysis Tools developed as part of the LDRD

V. Kunin, J. Raes, J.K. Harris, J.R. Spear, J.J. Walker, N. Ivanova, et al; "Millimiter-scale genetic gradients and community-level molecular convergence in a hypersaline microbial mat," Molecular Systems Biology 4, 198 (2008).

M.G. Kalyuzhnaya, A. Lapidus, N. Ivanova, A.C. Copeland, A.C. McHardy et al; "Highresolution metagenomics targets major functional types in complex microbial communities. Nature Biotechnolog, doi:10.1038/nbt.1488 (2008). 


\section{Presentations of the Metagenome Data Management and Analysis Tools}

-; "The Integrated Microbial Genomes (IMG) Family of Systems," Frontiers in Genomics seminar, National University of México, Campus Cuernavaca, Mexico, March 2008.

—; "Comparative Analysis and Annotation with the Integrated Microbial Genomes (IMG) Systems," plenary presentation at the Annual Meeting of the Israeli Society for Microbiology, Rehovot, Israel, April 2008.

-; "An Overview of IMG and IMG/M systems," Metagenome Sequence Data Management and Analysis, Metagenomics 2008 Conference, San Diego, Nov. 2008.

\section{CS-Rotem, Otoo LB08015 Energy-Smart Disk-Based Mass Storage System}

E. Otoo, A. Pinar, D. Rotem, S.-C. Tsao; "A File Allocation Strategy for Energy-Efficient Disk Storage Systems," LBNL Technical Report No LBNL-637E, July, 2008.

E. Otoo, D. Rotem, S.-C. Tsao; "Analysis of Trade-Off Between Power Saving and Response Time in Disk Storage Systems," submitted to High-Performance, Power-Aware Computing (HPPAC'09), 2009, Rome, Italy.

E. Otoo, D. Rotem, S.-C. Tsao; "Energy Smart Management of Scientific Data," in preparation to be submitted to Scientific and Statistical Database Management (SSDBM'09), 2009, New Orleans, Louisiana.

CS-Shalf et al LB08002 Holistic Approach to Energy Efficient Computing Architecture M. Mohiyuddin, M. Murphy, S. Williams, L. Oliker, J. Shalf, J. Wawrynek; "A Case for Hardware/Software Co-Tuning for Power Efficient Scientific Computing," submitted to $14^{\text {th }}$ International Symposium on High-Performance Computer Architecture, Marriott City Center, Salt Lake City, Feb. 16-20, 2008

CS-Skinner LB07029 Integrated Performance Monitoring of Grid and HPC Workloads

A. Uselton, L. Oliker, D. Skinner; "Understanding End-to-End Parallel I/O Performance through Scalable Profiling," in preparation to be submitted to Supercomputing 2009.

CS-Strohmaier et al LB08036 Reference Benchmarks for the Dwarfs (Algorithms)

E. Strohmaier; "Generalized Utility Metrics for Supercomputers," in preparation to be submitted to ICS09 or ISC09.

CS-Wilkening LB06026 Extended First Order System Least Squares Finite Elements

J. Wilkening; "Extended least squares finite elements for elliptic systems on plane domains with corners and interface junctions, " in preparation to be submitted to J. Comput. Phys.; LBNL MATH-PUB-9, 12/2008.

J. Wilkening; "Computation of stable representations of corner singularities of elliptic systems in the plane," LBNL MATH-PUB-8, 11/2008; shorter version in preparation for submission to Comm. Pure. Appl. Math.

J. Wilkening; "A trust region method for the computation of quadrature rules for arbitrary families of functions," in preparation to be submitted to J. Comput. Phys.; LBNL MATHPUB-7, 11/2008. 
J. Wilkening; "An infinite branching hierarchy of time-periodic solutions of the BenjaminOno equation," submitted to Indiana Univ. Math. J., 11/2008; preprint identifier: arXiv:0811.4209.

D. Ambrose and J. Wilkening; "Global paths of time-periodic solutions of the Benjamin-Ono equation connecting arbitrary traveling waves," submitted to Comm. Appl. Math. Computational Sci., 11/2008; preprint identifier: arXiv:0811.4205.

J. Wilkening and J. Yu; "A local construction of the Smith normal form of a matrix polynomial submitted to Journal of Symbolic Computation," 9/2008; preprint identifier: arXiv:0809.2978.

M. Westdickenberg and J. Wilkening; "Variational particle scheme for the porous medium equation and for the system of isentropic Euler equations," submitted to ESAIM-Math. Model. Num, 7/2008; preprint identifier: arXiv:0807.3573.

D. Ambrose and J. Wilkening; "Time-periodic solutions of the Benjamin-Ono equation," submitted to J. Nonlinear Sci., 4/2008; preprint identifier: arXiv:0804.3623.

Jon Wilkening; "Practical error estimates for Reynolds' lubrication approximation and its higher order corrections," accepted for publication in SIAM J. Math. Anal.; arXiv:0706.4103.

ESD-Berryman LB07008 Applications of Adjoint Field Methods and Time-Reversal Data Processing to Inverse Problems in Electromagnetics, Seismics, and Ultrasonics

Manuscript in preparation.

ESD-Christensen LB08025 Lattice-Boltzmann Investigations of Isotopic Fractionation During Mineral Precipitation

G. Lu, D. DePaolo, Q. Kang, D. Zhang; "Lattice Boltzmann simulation of snow crystal growth in clouds," accepted for publication in Journal of Geophysical Research Atmospheres.

G. Lu, D. DePaolo, Q. Kang, D. Zhang; "Lattice Boltzmann simulation of calcite precipitation and isotope fractionation," Eos Trans. AGU 89(53), Fall 2008 Meeting of the American Geophysical Union, December 2008, abstract MR21B-1796.

G. Lu and D. DePaolo; "Lattice Boltzmann method for transport and chemical reactions: Reactive Boundary and Benchmark," in preparation for submission to Advances in Water Resources Journal.

ESD-Collins et al LB08004 Integrated Earth Systems Climate Modeling and Analysis ???

M.J. Iacono, J.S. Delamere, E.J. Mlawer, M.W. Shephard, S.A. Clough, W.D. Collins; "Radiative forcing by long-lived greenhouse gases: Calculations with the AER radiative transfer models," J. Geophys. Res. 113, article no. D13103 (2008); doi:10.1029/2008JD009944.

M. Iacono, W. Collins, P. Rasch; "Evaluating the Impact of RRTMG/McICA in the NCAR CAM3.5 Climate Model," ARM Science Team Meeting, Mar. 10-14, 2008, Norfolk, Virginia. 
ESD-Holman LB06009 Compositional and Functional Analysis of Cell Walls During MetalBacterial Interactions

K.N. Woods, H.-Y.N. Holman, H. Wiedemann; "Solvent activated fluctuations and the Collective librational dynamics of lysozyme studied by THz spectroscopy," in preparation.

ESD-Jannson-C. LB08033 Maximizing Photosynthetic Yield by Increasing Sink Strength

E. Mangelsen, D. Wanke, J. Kilian, E. Sundberg, K. Harter, C. Jansson; "Diurnal fluctuations of transcript levels in developing barley caryopses - a microarray-based analysis," in preparation.

J. Mutisya, C. Sun, S. Rosenquist, C. Jansson; "Endogenous oscilation of SBE expression in sorghum endosperm," accepted for publication in J. Plant Physiol.

E. Mangelsen, J. Kilian, K.W. Berendzen, Ü, Kolukisaoglu, K. Harter, C. Jansson, D.Wanke; "Phylogenetic and comparative gene expression analysis of barley (Hordeum vulgare) WRKY transcription factor family reveals putatively retained functions between monocots and dicots," BMC Genomics 9, doi:10.1186/1471-2164-9-194 (2008).

Y. Baguma, C. Sun, M. Borén, H. Olsson, S. Rosenquist, J. Mutisya, P. Rubaihayo, C. Jansson; "Sugar-mediated semidian oscilation of gene expression in the cassava storage root regulates starch synthesis," Plant Sign. Behavior 3, 439-445 (2008).

C. Sun, H. Ghebramedhin, A.-S. Höglund, C. Jansson; "Antisense oligodeoxynucleotide inhibition as a potent diagnostic toold for gene function in plant biology," Plant Sign. Behavior 3, 328-330 (2008).

J. Hammargren, S. Rosenquist, C. Jansson, C. Knorpp; “A novel connection between nucleotide and carbohydrate metabolism in mitochondria: The Arabidopsis Nucleoside Diphosphate Kinase 3a gene is regulated by sugars," Plant Cell Rep. 27, 529-534 (2008).

ESD-Jansson-J. LB08034 Microbiomics of Complex Microbial Communities in Environmental Samples

Z. Wu, B. Willing, J. Bjerketorp, J.K. Jansson, K. Hjort; "Soft Inertial Microfluidics for High Throughput Separation of Bacteria From Human Blood Cells," in press Lab on a Chip.

J. Dicksved, M. Lindberg, M. Rosenquist, H. Enroth, J.K. Jansson, L. Engstrand; "Molecular Characterization of the Stomach Microbiota in Patients With Gastric Cancer and Controls," in press Journal of Medical Microbiology.

B. Willing, J. Halfvarson, J. Dicksved, M. Rosenquist, G. Järnerot, L. Engstrand, C. Tysk, J.K. Jansson; "Twin Studies Reveal Specific Imbalances in the Mucosa-Associated Microbiota of Patients With Ileal Crohn's Disease," in press Inflammatory Bowel Diseases.

W.-T. Liu and J.K. Jansson (eds); in press Environmental Molecular Microbiology, Norfolk, UK: Caister Academic Press.

J. Prosser, J.K. Jansson, W.-T. Liu; "Nucleic Acid-Based Characterization of Community Structure and Function," in press Environmental Molecular Microbiology, W.-T. Liu and J.K. Jansson, eds. Norfolk, UK: Caister Academic Press.

J.K. Jansson, J. Dicksved, L. Zhou; "The Human Environment," in press Environmental Molecular Microbiology, W.-T. Liu and J.K. Jansson, eds. Norfolk, UK: Caister Academic Press. 
N.C. Verberkmoes, A.L. Russell, M. Shah, A. Godzik, M. Rosenquist, J. Halfvarson, M.G. Lefsrud, J. Apajalahti, C. Tysk, R.L. Hettich, J.K. Jansson; "Shotgun Metaproteomics of the Human Distal Gut Microbiota," in press The ISME Journal.

A. Edlund and J.K. Jansson; "Use of Bromodeoxyuridine Immunocapture to Identify Psychrotolerant Phenanthrene-Degrading Bacteria in Phananthrene-Enriched Polluted Baltic Sea Sediments," FEMS Microbiology Ecology 65:513-525 (2008).

A. Edlund, F. Hårdeman, J.K. Jansson, S. Sjöling; “Active Bacterial Community Structure Along Vertical Redox Gradients in Baltic Sea Sediment," Environmental Microbiology 10:2051-2063 (2008).

J. Dicksved, J. Halfvarson, M. Rosenquist, G. Järnerot, C. Tysk, J. Apajalahti, L. Engstrand, J.K. Jansson; "Molecular Analysis of the Gut Microbiota of Identical Twins With Crohn's Disease," The ISME Journal 2: 716-727 (2008).

J.D. Van Elsas, J. Jansson, S. Sjoling, M. Bailey, R. Nalin, T. Vogel, R. Costa and L.V. Overbeek. The Soil Metagenome - A Recalcitrant, Yet Explorable Goldmine For Gene Hunters. Trends in Biotechnology. 2008. 26:591-601.

L. Jäderlund, V. Arthurson, U. Granhall, J.K. Jansson; "Specific Interactions Between Arbuscular Mycorrhizal Fungi and Plant Growth-Promoting Bacteria as Revealed By Different Combinations," FEMS Microbiology Letters 63:156-168 (2008).

J. Bjerketorp, A.N.T. Chiang, K. Hjort, M. Rosenquist, W-T. Liu, J.K. Jansson; "Rapid LabOn-A-Chip Profiling of Human Gut Bacteria," Journal of Microbiological Methods 72:82-90 (2008).

M. Unell, P.E. Abraham, M. Shah, B. Zhang, N.C. VerBerkmoes, J.K. Jansson, 'Impact of Phenolic Substrate and Growth Temperature on Arthrobacter chlorophenolicus Proteome," submitted to J. Proteome Research.

J.K. Jansson, B.Willing, A. Fekete, M. Lucio, P. Schmitt-Kopplin; "The Metametabolome of Twins With Crohn's Disease Unveils Potential Disease Biomarkers," in preparation for submission to Science.

K. Hjort, A. Bergstrom, A. Adesina, J.K. Jansson, K. Smalla, S. Sjoling, "Comparison of Chitinase and 16S rRNA Gene Diversity in Suppressive Soil Isolates, DNA Extracts and a Metagenomic Library," in preparation for submission to Environmental Microbiology.

L. Jaderlund, M. Hellman, K. Hornaeus, M. Che, L. Bo, J.K. Jansson, "Monitoring Colonization of Marker Gene-Tagged Plant Pathogen Ralstonia solanacearum and Plant Beneficial Pseudomonas fluorescens SBW25 on Tomato and Potato," in preparation for submission to FEMS Microbiology Ecology.

ESD-Nakagawa LB06017 New Technology for Permeability Enhancement for Natural Gas Extraction in Tight Reservoirs

S. Nakagawa and R.W. Ewy; "Shaped-core uniaxial compression test for studying borehole breakout in rocks," in Proceedings for American Rock Mechanics Association Meeting, Paper No.158, San Francisco, July 2008.

ESD-Oldenburg LB06018 Coupled Modeling of Hydrology, Nutrient Cycling, and Vegatation: Applications to Water Quality and Water Balance

C. Gu, F. Maggi, W.J. Riley, G.M. Hornberger, R.T. Venterea, T. Xu, N. Spycher, C. Steefel, N.L. Miller, C.M. Oldenburg, "Aqeuous and gaseous nitrogen losses induced by fertilizer application," in press J. of Geophysical Research-Biogeosciences; doi:10.1029/2008JG000788. 
C. Gu, F. Maggi, W. J. Riley, C.M. Oldenburg, N.M. Miller, N.M; “A Coupled Land Surface-Subsurface Biogeochemical Model for Watershed-scale Nitrogen Loss," CMWR Meeting 2008. San Francisco CA.

F. Maggi, C. Gu, W.J. Riley, G.M. Hornberger, R.T. Venterea, T. Xu, N. Spycher, C. Steefel, N.L. Miller, C.M. Oldenburg, "Mechanistic modeling of nitrogen cycling and losses," $J$. of Geophysical Research - Biogeosciences 113, G02016 (2008); doi:10.1029/2007JG000578.

F. Maggi and W.J. Riley; "Transient competitive complexation in biological kinetic isotope fractionation explains non-stationary isotopic effects: Theory and application to denitrification in soils," submitted to Journal of Geophysical Research - Biogeosciences.

F. Maggi and W.J. Riley; "Modeling Non-Steady Isotopic Effects Caused by Biological Kinetic Transient Complexation During Denitrification in Soils," AGU Fall Meeting 2008, San Francisco CA.

F. Maggi, C. Gu, W.J. Riley, Tianfu Xu, C.M. Oldenburg; "Nitrogen Cycle Modeling: Mechanistic Estimate of N-losses From Agricultural Fields on Seasonal Time Periods," Computational Methods in Water Resources, CMWR2008, San Francisco CA.

ESD-Perry LB08032 Design and Properties of Materials Involving Elements with High Neutron Cross-Sections... Useful in Neutron Detection ???

D.L. Perry and L.A. Franks; "Model Compounds for Cadmium [Neutron Sensitive] Zinc Telluride (CZT)," SPIE 7079, $70791 \mathrm{G}$ (2008).

A. Glatfelter, C. Dybowski, S. Bai, D. Kragten, M.J. Blake, S. Segarra, D.L. Perry; "Infrared Studies of Lead(II) Halide [Alternate Matrix Host Material for Neutron-Sensitive Metal Ions] - 1,10 - Phenanthroline Photosensitive Materials," Spectrochimica Acta A 71, 1922 (2009).

G.R. English, R.B. Firestone, D.L. Perry, J.P. Reijonen, K.-N. Leung, G.F. Garabedian, G.L. Molnar, Z.Revay; "Prompt Gamma Activation Analysis (PGAA) and Short-Lived Neutron Activation Analysis (NAA) Applied to the Characterization of Legacy Materials," J. Radioanal. Nucl. Chem. 277, 25 (2008).

Z. Ma, L.Liu, K.M. Yu, W. Walukieswicz, D.L. Perry, P.Y. Yu, S.S. Mao; "Experimental and Theoretical Studies on Gadolinium [Neutron Sensitive] Doping in ZnTe," J. Appl. Phys. 103, 023711 (2008).

EETD-Destaillats et al LB07014 Understanding the Chemistry of Innovative Air Cleaning $\underline{\text { Technologies }}$

Peer-reviewed journal articles

D. Kibanova, M. Trejo, H. Destaillats and J. Cervini-Silva; "Synthesis of clay-TiO, nanocomposites with photocatalytic activity for the degradation of air pollutants," Applied Clay Science 42, 563-568 (2009).

D. Kibanova, J. Cervini-Silva and H. Destaillats; "Efficiency of clay- $\mathrm{TiO}_{2}$ nanocomposites on the photocatalytic elimination of a model hydrophobic air pollutant," submitted to Environmental Science \& Technology.

A.T. Hodgson, H. Destaillats, T. Hotchi, D.P. Sullivan, W.J. Fisk; "Performance of ultraviolet photocatalytic oxidation (UVPCO) and combined UVPCO/chemisorbent air cleaning technologies under realistic indoor conditions," in preparation for submission to Ind. Eng. Chem. Res. 
N. Quici, M.L. Vera, H. Choi, D.D. Dionysiou, M.I. Litter, H. Destaillats; "Effect of key parameters on the photocatalytic oxidation of toluene in air under UVC irradiation," in preparation for submission to Applied Catalysis B-Environmental or to J. Photochem. Photobiol. A-Chemistry.

\section{Conference proceedings}

H. Destaillats, N. Quici, D. Kibanova, M.L. Vera, H. Choi, J. Cervini-Silva, D.D. Dionysiou, M.I. Litter; "Elimination of indoor air pollutants by photocatalytic oxidation: key parameters and challenges," in Proceedings of the $13^{\text {th }}$ international conference on $\mathrm{TiO}_{2}$ photocatalysis ( $\left.\mathrm{TiO}_{2}-13\right)$, San Diego, CA, September 2008.

A.T. Hodgson, H. Destaillats, T. Hotchi, W.J. Fisk; "Benefits and technological challenges in the implementation of TiO2-based ultraviolet photocatalytic oxidation (UVPCO) air cleaners," in Proceedings of the $11^{\text {th }}$ International Conference on Indoor Air Quality and Climate (Indoor Air 2008), Copenhagen, Denmark, August 2008.

N. Quici, D. Kibanova, M.L. Vera, H. Choi, D.D. Dionysiou, M.I. Litter, J. Cervini-Silva, A.T. Hodgson, H. Destaillats; "Investigation of key parameters influencing the efficient photocatalytic oxidation of indoor volatile organic compounds (VOCs)," Proceedings of the $11^{\text {th }}$ International Conference on Indoor Air Quality and Climate (Indoor Air 2008), Copenhagen, Denmark, August 2008.

N. Quici, M.L. Vera, H. Choi, D.D. Dionysiou, M.I. Litter, H. Destaillats; "Descomposición fotocatalítica de tolueno en aire. Efecto de parámetros críticos en el diseño de purificadores de aire," in Proceedings of the V Congreso Iberoamericano de Química y Física Ambiental, Mar del Plata, Argentina, April 2008.

EETD-Haves LB08037 Development of a Building Operating Platform

F. Balarin, M. D’Angelo, A. Davare, D. Densmore, T. Meyerowitz, R. Passerone, A. Pinto, A. Sangiovanni-Vincentelli, A. Simalatsar, Y. Watanabe, G. Yang, Q. Zhu; "Platformbased design and frameworks: Metropolis and Metro II," in Model-based design of heterogeneous embedded systems, G. Nicolescu, P. Mosterman (Eds.), CRC Press, to appear Spring 2009.

EETD-Levine LB07036 China's Energy Future: Changes in Energy Intensity

J. Lin, N. Zhou, M. Levine, D. Fridley; "Taking out one billion tons of CO2: the magic China's 11th five-year plan?" in Energy Policy 36, 954-970 (2008).

M. Levine and N. Aden; "Global Carbon Emissions in the Coming Decades: The Case of China," Annual Review of Environment and Resources 33 (November 2008); LBNL$372 \mathrm{E}$.

EETD-McMahon et al LB07021 Integrated Decision Support Tool for Joint Optimal Control of Energy and Water Systems

Publications and Papers

P. Schwartz, and P. Colella; "A Second-Order Accurate Method for Solving the Eikonal Equation," in press Communications in Applied Mathematics and Computational Science; $\leq$ http://seesar.lbl.gov/anag/publications/colella/EikonalEquation-2008.pdf $>$. 
N. L. Miller, L. L. Dale, S. D. Vicuna, T. N. Kadir, E. C. Dogrul, C. F. Brush; "Drought Analysis of the California Central Valley Surface-Groundwater-Conveyance System," accepted for publication in Journal of American Water Resources Association.

B. Faybishenko, et. al; "Evaluation of the Epistemic Uncertainty of Evapotranspiration and Groundwater Recharge Using Maximum Likelihood Bayesian Averaging," in preparation for submission to Water Resources Research.

\section{Presentations and Conference Papers}

L. Dale, S.Vicuna, B.Faybishenko; "Modeling the impact of energy and water prices on reservoir and aquifer management," American Geophysical Union Fall Meeting, /Eos Trans, December 18 2008, San Francisco CA.

L. Dale; "Case studies in American and Merced River Basins. Climate Change and Energy in California," California Energy Commission Seminar, April 2008.

L. Dale, N. Miller, C. Bush, T. Kadir; "Impact of Climate Change on San Joaquin Valley Water Supply and Potential Agricultural Adaptation Strategies," Fifth Annual California Climate Change Research Conference, September 2008.

L. Dale, M. Hanemann, S. Vicuna, J. Dracup, S. Fujita; "Impact of Changes in Household Energy and Water Rates, Rate Structures," Impact of Climate Change on Groundwater and Reservoirs, $21^{\text {st }}$ Annual Western Conference, Center for Research in Regulated Industries, Monterey, June 2008.

L. Dale; "Impact to California Groundwater Levels and Cropping Patterns Resulting from Climate Change and Drought Conditions," Applications of Optimization Techniques to Groundwater Projects," Groundwater Resources Association, Sacramento, October 16 2008.

P. Schwartz, and P. Colella; "A Second-Order Accurate Method for Solving the Eikonal Equation," to appear in Communications in Applied Mathematics and Computational Science; http://seesar.lbl.gov/anag/publications/colella/EikonalEquation-2008.pdf.

B. Faybishenko and S.P. Neuman; "Quantifying epistemic and parameter uncertainties in predictions of evaporation and net infiltration, using maximum likelihood Bayesian model averaging," Geophysical Research Abstracts 10, EGU2008-A-00000, European Geosciences Union General Assembly, April 2008, Vienna, Austria.

B. Faybishenko; "Fuzzy systems modeling for uncertainty predictions in water resources," abstract for Computational Methods in Water Resources, XVII International Conference, July 6-10 2008, San Francisco CA.

L.S. Dale, S. Vicuna, J. A. Dracup; "The Conjunctive Use of Reservoirs and Aquifers: tradeoffs in electricity generation and water supply," ASCE EWRI Congress. May 2008. Honolulu HI.

N.L. Miller; "Climate Change Impacts on the SF Bay-Delta and Central Valley Water System," California Climate Change and Water Adaptation Summit, Santa Monica CA, October 2007.

N.L. Miller; "Large-Scale Surface-Groundwater Modeling: A Partial Review with Example," Workshop on Global Monitoring of Groundwater Resources, Utrecht, The Netherlands, October 2007.

N.L. Miller, "Climate Change and Fresh Water Impacts," NOAA National Marine Fisheries Service Workshop, Tahoe City CA, August 2008.

N.L. Miller, L L. Dale, C Bush, S Vicuna, T Kadir, J Drogul, F. Chung; "Drought Scenario Analysis of the California Central Valley Surface-Groundwater-Conveyance System," 
Groundwater Association of California, Climate Change: Implications for California Groundwater Management, Sacramento CA, August 2008.

N.L. Miller; "Global Warming and Its Effects on California," Symposium on Green Innovations for a Clean California, April 2008.

N.L. Miller; "Water Cycle Measurement Uncertainties and Droughts Analysis in California," IGCP 565 Project Workshop, San Francisco CA, December 2008.

EETD-Rubinstein LB08039 Using IP Telephony and Wireless Technologies to Extend the Reach of Conventional Building Automation Systems

J Granderson, F Rubinstein, D DiBartolomeo, G Ghatikar; "The Performance of an Integrated Dimmable Lighting and Motorized Venetian Blind System," in preparation to be submitted to Leukos.

EETD-Wetter LB08041 Building Informatics Environment Enabling Rapid Prototyping and Model Extraction for Building Automation Systems

M. Wetter; "Modelica-based Modeling and Simulation to Support Research and Development in Building Energy and Control Systems," submitted to Journal of Building Performance Simulation.

M. Wetter; "The Future of Building Modeling and Simulation," to appear in Building Performance Simulation for Design and Operation, Jan L.M. Hensen and Roberto Lamberts (eds), Taylor and Francis Group.

GN-Eisen LB06033 Computational and Experimental Testing of Methods for Binning Sequences from Metagenomic Studies

S. Chatterji, I. Yamazaki, Z. Bai, JA Eisen; "CompostBin: A DNA composition-based algorithm for binning environmental shotgun reads," RECOMB 2008.

J. Morgan, A. Darling, JA Eisen; "Shotgun Sequencing of an in vitro-Simulated Microbial Community," in preparation.

M. Huntemann, JA Eisen; "OTUHunter: software for clustering DNA sequences for microbial diversity studies," in preparation for submission to BMC Bioinformatics or PLoS One.

LSD-Butland LB07010 Functional Interactomics: Integrating Physical and Functional Interaction Networks

G. Butland, M. Babu, J.J. Díaz-Mejía, et al; "eSGA: E. coli Synthetic Genetic Array analysis," Nature Methods, August $1^{\text {st }} 2008$ (online); http://www.nature.com/nmeth/journal/v5/n9/abs/nmeth.1239.html.

LSD-Hang LB08027 Interaction of Fragile X Mental Retardation Protein with Thymine-DNA Glycosylase:Implication in the Molecular Mechanism of Fragile X Syndrome

B. Hang, H. Budworth, A.H. Sarker, P.K. Cooper; "Functional interaction of human TDG with FMRP and XPG in base excision repair of T/G mismatches," Environmental and Molecular Mutagenesis 49, 546 (2008). 
LSD-Wyrobek LB06035 Expression Profiling of Radiation and Cancer Susceptibility Genes

T. Straume, S.A. Amundson, W.F. Blakely, F.J. Burns, A. Chen, N. Dainiak, S. Franklin, J.A..Leary, D.J. Loftus, W.F. Morgan, T.C. Pellmar, V. Stolc, K.W. Turteltaub, A.T. Vaughan, S. Vijayakumar, A.J. Wyrobek; "Meeting Report ---NASA Radiation Biomarker Workshop," in press Radiation Research.

X.R. Lowe, X. Lu, F. Marchetti, A.J. Wyrobek;"'The expression of Troponin T1 gene is induced by ketamine in adult mouse brain," Brain Res. 2007 Oct 12;1174:7-17.

X.R. Lowe, S. Bhattacharya, F. Marchetti, A.J. Wyrobek' "Early brain response to low-dose radiation exposure involves molecular networks and pathways associated with cognitive functions, advanced aging, and Alzheimer's disease," in press Radiation Res.

MSD-Javey LB06038 Integration of Synthetic Nanomaterials for High Performance, Robust, and Flexible Circuitry

Z. Fan, J. C. Ho, Z. A. Jacobson, H. Razavi, A. Javey; "Large Scale, Heterogeneous Integration of Nanowire Arrays for Image Sensor Circuitry," Proceedings of the National Academy of Sciences (PNAS) 105, 11066-11070 (2008).

A. C. Ford, J. C. Ho, Z. Fan, O. Ergen, V. Altoe, S. Aloni, H. Razavi, A. Javey; "Synthesis, Contact Printing, and Device Characterization of Ni-Catalyzed, Crystalline InAs Nanowires," Nano Research 1, 32-39 (2008).

J. C. Ho, R. Yerushalmi, Z. A. Jacobson, Z. Fan, R. L. Alley, A. Javey; "Controlled nanoscale doping of semiconductors via molecular monolayers," Nature Materials 7(1), 62-67 (2008).

Z. Fan, J. C. Ho, Z. A. Jacobson, R. Yerushalmi, R. L. Alley, H. Razavi, A. Javey; "WaferScale Assembly of Highly Ordered Semiconductor Nanowire Arrays by Contact Printing," Nano Letters 8(1), 20-25 (2008, cover article).

\section{MSD-Jérôme LB08029 Transport in Thin Polymer Films}

M. Mezger, B. Jérôme, J.B. Kortright, M. Valvidares, E.M. Gullikson; "Resonant Soft X-ray Reflectivity Studies on the Structural Arrangement of Polymers at Interfaces," to be presented at APS March Meeting 2009.

MSD-Long LB08030 Conducting Metal-Organic Frameworks

J. Behera, D. D'Alessandro, N. Soheilnia, J. Long; "Synthesis and characterization of allruthenium and iron-ruthenium Prussian blue analogues," in preparation for submission to Chemistry of Materials.

MSD-Ritchie LB08049 Fracture of Cortical Bone: Mechansims Underlying the Origins of Toughness in Hard Mineralized Tissues

K.J. Koester, J.W. Ager III, R.O. Ritchie; "Aging and Fracture of Human Cortical Bone and Tooth Dentin," Journal of Materials 80(6), 33-38 (2008).

W. Yao, Z. Cheng, A. Pham, C. Busse, E. Zimmerman, R.O. Ritchie, N.E. Lane; "Glucocorticoid-Induced Bone Loss in Mice Can Be Reversed by the Actions of Parathyroid Hormone and Risedronate on Different Pathways for Bone Formation and Mineralization," Arthritis and Rheumatism 58(11), 3485-3497 (2008). 
MSD-Saiz LB08045 Three Dimensional Printing of Ceramics with Submicron Resolution

J. Franco, P. Hunger, E. Munch, E. Saiz, A.P. Tomsia; "Synthesis of porous ceramic materials," in preparation for submission to International Journal of Materials Research.

J. Franco, A.P. Tomsia, E. Saiz; "Three dimensional printing of highly porous ceramic and metal-ceramic nanocomposites," in preparation for submission to Advanced Materials.

MSD-Stamper-Kurn LB07030 Two-Element Ultracold-Atom Quantum Simulator of Materials

M. Vengalattore, S.R. Leslie, J. Guzman, D.M. Stamper-Kurn; "Spontaneously modulated spin-textures in a dipolar spinor Bose-Einstein condensate," Phys. Rev. Lett. 100, 170403 (2008).

M. Vengalattore, J. Guzman, S.R. Leslie, F. Serwane, D.M. Stamper-Kurn; "Crystalline magnetic order in a dipolar quantum fluid," submitted for review.

S.R. Leslie, J. Guzman, M. Vengalattore, J.D. Sau, M.L. Cohen, D.M. Stamper-Kurn; "Amplification of fluctuations in a spinor Bose-Einstein condensate," submitted for review; preprint arXiv:0806.1553.

MSD-Wu LB08024 Transition-Metal-Doped GeTe Nanowires as a Single-Phase Multiferroic System

J. W. L. Yim, B. Xiang, J. Wu; "In situ TEM Investigation of Solid-Liquid-Vapor Phase Transition in GeTe Nanowires," MRS Spring Meeting, San Francisco, March 24-28 2008.

J. Wu, J. Cao, J. W. L. Yim, B. Xiang; "Phase Transitions at the Nanoscale," MRS Fall Meeting, Boston, Dec. 1-5 2008.

B. Xiang, D. R. Khanal, F. Wong, J. Wu; "Direct Observation of Conductive Filaments in Planar Resistance Switching Devices, in preparation for submission to Applied Physics Letters, 2008.

MSD-Xu LB08042 Hierarchical Assemblies of Peptide-Polymer Conjugates

J. Shu, C. Tan, W. F. DeGrado, T. Xu; “A New Design of Helix Bundle Forming PeptidePolymer Conjugate," Biomacromolecules 9, 2111 (2008);

http://pubs.acs.org/doi/full/10.1021/bm800113g.

NSD-Clark LB07012 New Experimental Initiative to Deduce (n,f) Cross Sections for Advanced Fuel Studies

B. F. Lyles, L. A. Bernstein, J. T. Burke, F. S. Dietrich, J. Escher, I. Thompson, D. L. Bleuel, R. M. Clark, P. Fallon, J. Gibelin, A. O. Macchiavelli, M. A. McMahan, L. Phair, E. Rodriguez-Vieitez, M. Wiedeking, C. W. Beausang, S. R. Lesher, B. Darakchieva, M. Evtimova; "Absolute and relative surrogate measurements of the $236 \mathrm{U}(\mathrm{n}, \mathrm{f})$ cross section as a probe of angular momentum effects," Phys. Rev. C 76, 014606 (2007).

S. R. Lesher, J. T. Burke, L. A. Bernstein, H. Ai, C. W. Beausang, D. L. Bleuel, R. M. Clark, F. S. Dietrich, J. E. Escher, P. Fallon, J. Gibelin, B. F. Goldblum, I. Y. Lee, A. O. Macchiavelli, M. A. McMahan, K. J. Moody, E. B. Norman, L. Phair, E. RodriguezVieitez, N. D. Scielzo, M. Wiedeking; "The Surrogate Ratio Method in the Actinide Region Using the $\left(\alpha, \alpha^{\prime}\right.$ ) Reaction," accepted for publication in Phys. Rev. C. 
NSD-Lesko, Wang LB07020 Physics Detector and Sensor Technologies Applied to Geological and Geophysical Applications at DUSEL

J.S. Wang, "The Role of Sandlines between Levels in the DUSEL Study: Carbon in Hydrological Environment for Enhanced Retention and Sequestration," Annual Geophysical Union Meeting, San Francisco, December 2008.

P. Luke, M. Ammon, Y.D.Chan, J. Detwiler, M. Bergevin, K.T.Lesko; "Point Contact Detector Design," in preparation for presentation at IEEE 2008.

NSD-Sichtermann LB07027 Hyperons in Polarized Proton Collisions and the Origin of the Nucleon Spin

Y. Chen, Z.T. Liang, E.P. Sichtermann, Q.H. Xu, S.S. Zhou; "Anti-Hyperon Polarization in High Energy pp Collisions with Polarized Beams," Phys. Rev. D 78, 054007 (2008); LBNL-63097.

B.I. Abelev et al [STAR Collaboration]; paper on jet trigger results in preparation for submission to Phys. Rev. D.

NSD-Vetter LB06025 Development of a Low-Energy, High-Current Astrophysics Accelerator

D.S. Todd, D. Leitner; "Design of variable acceleration gap for a high current, low-emittance ion acceleration column," in preparation for submission to Nuclear Instruments and Methods A.

PBD-Crooks LB08005 Understanding the Nanothermodynamics of Molecular Machines

G.E. Crooks; "Quantum operation time reversal," Phys. Rev. A 77(3):034101(4) (2008).

P. Maragakis, F. Ritort, M. Karplus, C. Bustamante, G.E. Crooks; "Bayesian estimates of free energies from nonequilibrium work data in the presence of instrument noise," $J$. Chem. Phys. 129, 024102 (2008).

E.H. Feng and G.E. Crooks; "Length of time's arrow," Phys. Rev. Lett. 101(9):090602 (2008).

G.E. Crooks; "On the Jarzynski relation for dissipative quantum dynamics," J. Stat. Mech.: Theor. Exp. 2008(10):P10023 (9pp).

PBD-Liphardt et al LB08013 Light-Boosted Fermentation in the Yeast Saccharomyces Cervisiae

J. Walter, J. Triffo, M. Auer, J. Liphardt; "Design and Synthesis of Artificial Chloroplasts in S. Cerevisiae," in preparation for submission to PNAS or one of Nature family.

PBD-Schaffer et al LB07024 Cooperation of Biochemical and Mechanical Signals in Regulating Cell Fate Decision During Tissue Morphogenesis

K. Saha, A.J. Keung, E.F. Irwin, Y. Li, L. Little, D.V. Schaffer, K.E. Healy; "Substrate Modulus Directs Neural Stem Cell Behavior," Biophysical Journal 95:4426-4438 (2008).

L. Little, D.V. Schaffer, K.E. Healy; "Engineering Biomaterials for Synthetic Neural Stem Cell Microenvironments," Chemical Reviews 108:1787-1796 (2008). 
S.T. Wall, K. Saha,R.S. Aston, K.R. Kam, D.V. Schaffer, K.E. Healy; "Multivalency of Sonic Hedgehog Conjugated to Linear Polymer Chains Modulates Protein Potency," Bioconjugate Chemistry 19:806-812 (2008).

S. Agrawal, C. Archer, D.V. Schaffer; "Computational Models of the Notch Network Elucidate Mechanisms of Context-dependent Signaling," revised manuscript submitted to Public Library of Science (PLoS) Computational Biology.

C. Pangarkar, R. Ashton, D. Schaffer; "EphrinB2 Regulates the Neuronal Differentiation of Adult Neural Stem Cells," in preparation for submission to Nature Neuroscience or Journal of Neuroscience.

PD-Battaglia et al LB08028 Advanced Silicon Detectors for Future Short Pulse X-ray Sources

M. Battaglia et al.; "Monolithic Pixel Sensors in Deep-Submicron SOI Technology with Analog and Digital Pixels," to appear in Nucl. Instrum. Meth. A.

PD-Bauer LB07004 Soft-collinear Effective Theories Applied to Collider Physics

C.W. Bauer, O. Cata, G. Ovanesyan; "On different ways to quantize Soft-Collinear Effective Theory," e-Print: arXiv:0809.1099 [hep-ph].

C.W. Bauer, A. Hornig, F.J. Tackmann; "Factorization for generic jet production," e-Print: arXiv:0808.2191 [hep-ph].

C.W. Bauer, S.P. Fleming, C. Lee, G. Sterman; "Factorization of e+e- Event Shape Distributions with Hadronic Final States in Soft Collinear Effective Theory," Phys. Rev. D 78:034027 (2008).

C.W. Bauer, F.J. Tackmann, J. Thaler; "GenEvA. II. A Phase space generator from a reweighted parton shower," accepted for publication in Phys. Rev. D.

C.W. Bauer, F.J. Tackmann, J. Thaler; "GenEvA. I. A New framework for event generation," accepted for publication in Phys. Rev. D.

\section{PD-Garcia-Sciveres LB08007 Development of Nanowire Carpet Hybrid Pixel (NCHyP)}

$\underline{\text { Detectors }}$

M. Garcia-Sciveres and C. Tindall; "Method of fabrication of silicon pixel radiation sensors using nanowire etching," Record of Invention disclosure, 9/10/2008.

M. Garcia-Sciveres and C. Tindall; "Silicon pixel radiation sensors having nano-wire connectors and methods for fabrication of same," Provisional patent application 9/30/2008, received by US Patent Office 10/3/08 with application number 61102761 .

PD-Gilchriese, Haber LB08048 Development of Multi-Modular Assemblies with Reduced Material and Services for Specifications at Future Particle Tracking

M. Cepeda, S. Dardin, M. Gilchriese, W.K. Miller, W.O. Mille, R. Post; "Pixel Detector Local Mechanical Supports with Integrated Cooling Based on Thermally Conducting Carbon Foam," under review for ATLAS Internal Note.

E. Anderssen, M. Cepeda, S. Dardin, M. Garcia-Sciveres, M. Gilchriese, C. Haber, N. Hartman, W. Miller and W. Miller. R. Post; "Mechanical/Cooling Supports Based on Thermally Conducting Carbon Foam," presented at Pixel 2008 Conference, to be submitted to JINS. 
H. Chen et al [collaboration]; "Performance of Large Serially Powered Integrated Silicon Tracking Elements for the Super-LHC," presented at the 2008 IEEE Nuclear Science Symposium.

PD-Padmanabhan LB08046 Calibrating Baryon Acoustic Oscillations for Future Dark Energy Experiments

N. Padmanabhan, M. White; "Calibrating Baryon Oscillations I : The Nonlinear Clustering of Matter," in preparation to be submitted to Phys. Rev. D.

N. Padmanabhan, M. White, J. Cohn; "Reconstructing Baryon Oscillations : A Lagrangian Theory Perspective," in preparation to be submitted to Phys. Rev. D.

Y. Noh, N. Padmanabhan, M. White: "Quantifying the effect of sampling on baryon oscillation reconstruction," in preparation to be submitted to Phys. Rev. D.

PD-Roe et al LB08043 High-Yield CCD Process Development at the LBNL MicroSystems Lab

S. E. Holland et. al; "A 12.3 Mpixel, Fully Depleted, Back-Illuminated, High-Voltage Compatible Charge-Coupled Device for the SuperNova Acceleration Probe," to be submitted to IEEE Transactions on Electron Devices.

PD-Schlegel LB07025 Baryon Oscillations and Dark Energy: Prototyping Instruments

D. Schlegel, B. Ghiorso; "LBNL Fiber Positioners for Wide Field Spectroscopy," Proceedings of the SPIE: Advanced Optical and Mechanical Technologies in Telescopes and Instrumentation Vol 7018, June 2008.

PD-Smoot LB08020 Decoding Dark Energy with Weak Gravitational Lensing

Alexie Leauthaud et al; "A Weak Lensing Study of X-ray Groups in the COSMOS Survey: Constraining the Low Mass End of the Lx-M Relation," in preparation.

Cecile Faure et al; "On the contribution of large scale structure to strong gravitational lensing," submitted to $A p J$; http://adsabs.harvard.edu/abs/2008arXiv0810.4838F.

Olivier Ilbert et al; "COSMOS Photometric Redshifts with 30-bands for 2-deg2," accepted for publication in $A p J$; http://adsabs.harvard.edu/abs/2008arXiv0809.2101I.

Cecile Faure et al; "First Catalog of Strong Lens Candidates in the COSMOS Field," ApJs 176, 19-38 (May 2008); http://adsabs.harvard.edu/abs/2008ApJS..176...19F.

Y.Shioya et al; "The H-alpha Luminosity Function and Star Formation Rate at $\mathrm{z} \sim 0.24$ in the COSMOS 2 Square Degree Field," ApJs 175, 128-137 (March 2008); http://adsabs.harvard.edu/abs/2008ApJS..175..128S.

K. Caputi; "The Optical Spectra of 24-micron Galaxies in the COSMOS Field. I. Spitzer MIPS Bright Sources in the zCOSMOS-Bright 10k Catalog," ApJ 680, 939-961 (June 2008); http://adsabs.harvard.edu/abs/2008ApJ...680..939C.

Cross-Div.-Holbrook, Banfield LB08010 Do CRISPR Regions of Bacteria and Archaea Constitute an RNAi Based Immune System?

A. Andersson and J. F. Banfield; "Virus Population Dynamics and Acquired Virus Resistance in Natural Microbial Communities," Science 230, 1047-1050 (2008). 
B. Wiedenheft and J. Doudna; "Structural Basis for DNase Activity of a Conserved Protein Involved in CRISPR-Mediated Anti-Viral Defense," in preparation for submission to Structure.

Cross-Div.-Lee LB06031 Fabrication of Photovoltaic Devices Using Nano-Sructured Biomaterials

A. Merzlyak and S.-W. Lee; "Construction Rules of Major Coat Engineered M13 Phage," in preparation for submission to Biomacromolecules.

A. Merzlyak, S. Indrakanti, S.-W. Lee; "Genetically Engineered Nanofiber-Like Viruses For Tissue Regenerating Materials," submitted to Nano Letter.

Cross-Div.-Schroeder et al LB08018 Coupled Process Models and Monitoring for Advanced Nuclear Fuel Cycles

E. Blandford, L. Kim, M.Laufer, P. Peterson; "A Global Nuclear Energy Expansion Scenario: The World in 2030," to be published in Conference Proceedings for U.C. Berkeley Pathways Conference: Scenarios for Nuclear Energy and Nuclear Weapons in 2030 and Implications for United States Policy, September 18-19, 2008.

M. Laufer; "A General Framework for Using Simulation in the Licensing of Advanced Nuclear Energy Systems," in preparation.

M. Janousch, R. Copping, T. Tyliszczak, I. Castro-Rodriguez, D. K. Shuh; "Scanning transmission x-ray spectromicroscopy of actinide complexes," Mater. Res. Soc. Symp. Proc. 1104, 165-170 (2008).

B. Seon, A. Canning, N. Jensen; "First-principles studies of Pu and Ce Maltol and Br-Maltol complexes," in preparation to be submitted to Journal of Computational Physics.

B Quiter, V. Mozin, B. Ludewigt; "Nuclear Resonance Fluorescence for the Assaying of Spent Fuel," in preparation for possible submission to Journal of Nuclear Materials Management. 